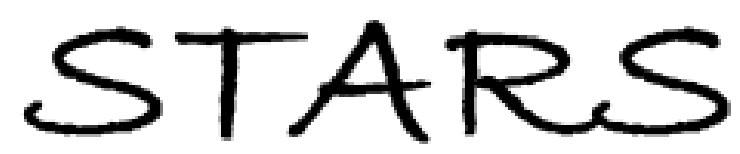

University of Central Florida

STARS

2013

\title{
Smooth And Non-smooth Traveling Wave Solutions Of Some Generalized Camassa-holm Equations
}

Taslima Rehman

University of Central Florida

Part of the Mathematics Commons

Find similar works at: https://stars.library.ucf.edu/etd

University of Central Florida Libraries http://library.ucf.edu

This Masters Thesis (Open Access) is brought to you for free and open access by STARS. It has been accepted for inclusion in Electronic Theses and Dissertations, 2004-2019 by an authorized administrator of STARS. For more information, please contact STARS@ucf.edu.

\section{STARS Citation}

Rehman, Taslima, "Smooth And Non-smooth Traveling Wave Solutions Of Some Generalized Camassaholm Equations" (2013). Electronic Theses and Dissertations, 2004-2019. 2682.

https://stars.library.ucf.edu/etd/2682

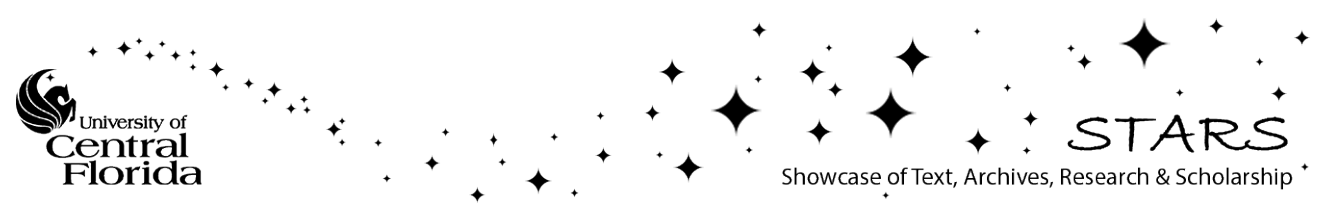




\title{
SMOOTH AND NON-SMOOTH TRAVELING WAVE SOLUTIONS OF SOME GENERALIZED CAMASSA-HOLM EQUATIONS
}

\author{
by
}

TASLIMA REHMAN

B.Sc. University of Dhaka, 2008

M.Sc. University of Dhaka, 2010

\begin{abstract}
A dissertation submitted in partial fulfillment of the requirements
for the degree of Master of Science

in the Department of Mathematics

in the College of Sciences

at the University of Central Florida

Orlando, Florida
\end{abstract}

Summer Term

2013

Major Professor: S. Roy Choudhury 
(C) 2013 Taslima Rehman 


\begin{abstract}
In this thesis we employ two recent analytical approaches to investigate the possible classes of traveling wave solutions of some members of recently derived integrable family of generalized Camassa-Holm (GCH) equations.
\end{abstract}

In the first part, a novel application of phase-plane analysis is employed to analyze the singular traveling wave equations of four $\mathrm{GCH}$ equations, i.e. the possible non-smooth peakon, cuspon and compacton solutions. Two of the GCH equations do no support singular traveling waves. We generalize an existing theorem to establish the existence of peakon solutions of the third GCH equation. This equation is found to also support four segmented, non-smooth M-wave solutions. While the fourth supports both solitary (peakon) and periodic (cuspon) cusp waves in different parameter regimes.

In the second part of the thesis, smooth traveling waves of the four GCH equations are considered. Here, we use a recent technique to derive convergent multi-infinite series solutions for the homoclinic and heteroclinic orbits of their traveling-wave equations, corresponding to pulse and front (kink or shock) solutions respectively of the original PDEs. Unlike the majority of unaccelerated convergent series, high accuracy is attained with relatively few terms. Of course, the convergence rate is not comparable to typical asymptotic series. However, asymptotic solutions for global behavior along a full homoclinic/heteroclinic orbit are currently not available. 
Dedicated to my Parents for constant support,

inspiration and for being beside me in tough times 


\section{ACKNOWLEDGMENTS}

First and foremost I would like to thank Almighty ALLAH for giving me the strength and making the completion of this thesis possible.

It has been a long journey full of ups and downs, lots of precious memories, and successes which are the result of help, suggestion and inspiration of many good friends, colleagues, and acquaintances. First of all, I would like to express my deep gratitude to my thesis advisor Dr. Sudipto Roy Choudhury, who has supported me throughout my thesis with his patience and knowledge whilst allowing me room to work in my own way. One simply could not wish for a better or friendlier advisor. I would also like to thank Dr. Gaetana Gambino (University of Palermo, Italy) for her constant support by checking my calculations, which really helped me in completion of my thesis. She also helped me in clearing up basics in many topics. I also want to thank Dr. Andrew Nevai and Dr. David Rollins for serving in my committee and managing time in their busy schedule.

Special thanks to Dr. Piotr Mikusinski (Chair Dept. of Mathematics, University of Central Florida) and Dr. Joseph Brennan (Graduate Coordinator, Dept. of Mathematics, University of Central Florida) for providing me with valuable advice throughout my Master's Program at UCF. 
During the period of two years, many friends were helpful to color my life. I have to acknowledge my friends and seniors for their assistance in many aspects that I cannot list them all. I am also grateful to Norma Robles (Graduate Program Assistant) because she reduces our burdens in dealing with administrative routines.

I owe more than thanks to my parents for their constant support, encouragement and everything they have done to help me succeed throughout my life. I would also like to thank my two lovely sisters, Bushra and Nusrat, who were always there for me whenever I needed them. They were like a mental support for me throughout my entire life. Last but not the least; I would like to say thanks to my beloved husband Dr. Muhammad Qazi for all the help he provided during my thesis preparation. He has been a source of inspiration for me during my whole Master's program. Without his support it would have been impossible for me to finish my graduate education. 


\section{TABLE OF CONTENTS}

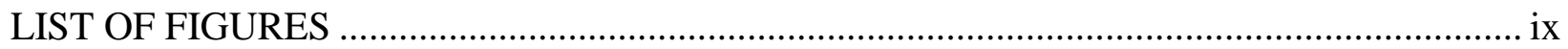

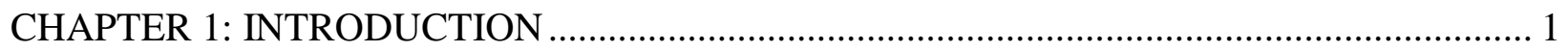

CHAPTER 2: BASIC MATHEMATICAL THEORY OF SINGULAR TRAVELLING WAVE

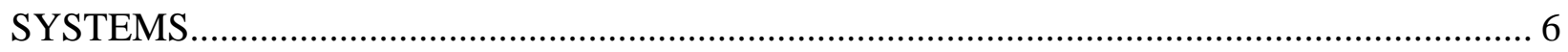

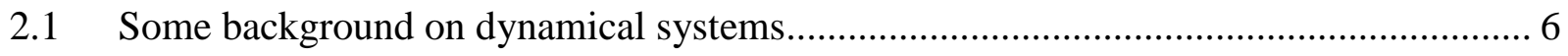

2.2 Phase portraits of traveling wave equations having singular straight lines..................... 9

2.2.1 The dynamics of the first type of singular traveling waves .................................... 9

2.2.2 Main theorems to identify the profiles of waves .............................................. 12

2.2.3 The dynamics of the second type of singular traveling waves ............................. 18

CHAPTER 3: PHASE PLANE ANALYSIS OF THE GENERALIZED CAMASSA-HOLM EQUATIONS: POSSIBLE SINGULAR AND REGULAR SOLUTIONS ................................ 20

3.1 Phase portrait/possible solutions of Equation (1.4) .................................................. 20

3.2 Phase portrait/possible solutions of Equation (1.5) ................................................... 25

3.3 Phase portrait/possible solutions of Equation (1.6).................................................... 29

3.4 Phase portrait/possible solutions of Equation (1.7) ..................................................... 36 


\section{CHAPTER 4: ANALYTIC SOLUTIONS FOR HOMOCLINIC AND HETEROCLINIC ORBITS OF GENERALIZED CAMASSA HOLM EQUATIONS ........................................... 41}

4.1 Infinite Series for heteroclinic orbits of Equation (3.2) ……….................................. 42

4.2 Infinite Series for homoclinic orbits of Equation (3.6) ………………...................... 52

4.3 Infinite Series for homoclinic orbits of Equation (3.12) ….......................................... 66

4.4 Infinite Series for homoclinic orbits of Equation (3.17) …….................................... 74

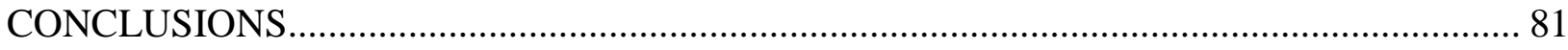

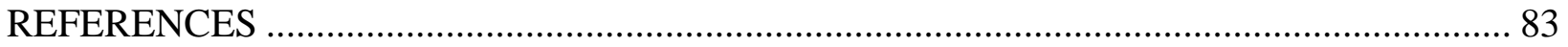




\section{LIST OF FIGURES}

Figure 1: Plot of $u(\mathrm{x}, \mathrm{t})$ with the change of $x$ and $t$ showing two peakons with different wave speed colliding with each other forming double peakon (Image taken from Reference [3])........ 2

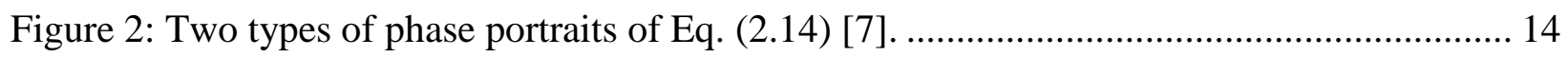

Figure 3: $\phi(\xi)$ and $\phi^{\prime}(\xi)$ plotted against $\xi$ corresponding to Figure 2.1 [7]......................... 15

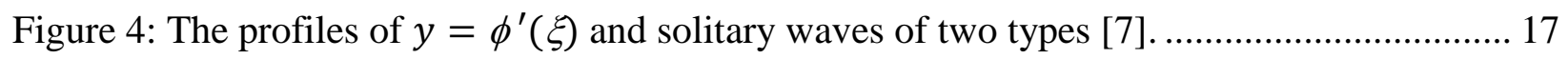

Figure 5: The phase portraits (y plotted against $\phi$ ) of Equation (3.4)..................................... 24

Figure 6: The phase portraits (y plotted against $\phi$ ) of Equation (3.8). Dotted line is the singular line $c+4 \phi-2 y=0$

Figure 7: The phase portraits (y plotted against $\phi$ ) of Equation (3.14)..................................... 35

Figure 8: The phase portraits (y plotted against $\phi$ ) of Equation (3.19) ................................. 40

Figure 9: The parameters are chosen as $\mathrm{c}=-2$ and $\mathrm{g}=0.2$. (a) Discontinuous series solution for the heteroclinic orbit joining $x_{1}=0.1127$ and $x_{2}=0.8873$. (b) The series coefficients $a_{k}$ converge (c) The series coefficient $b_{k}$ converge. 48

Figure 10: The parameters are chosen as $c=-2$ and $g=0.2$. (a) Series solution for the heteroclinic orbit joining $x_{1}=0.1127$ and $x_{2}=0.8873$. Here $\phi^{+}(0)=\phi^{-}(0)=0.8637$, (b) The series coefficients $a_{k}$ converge (c) The series coefficient $b_{k}$ converge.

Figure 11: The parameters are chosen as $c=3$ and $g=1.1$. (a) Discontinuous series solution for the heteroclinic orbit joining $x_{1}=-0.6382$ and $x_{2}=-0.8612$. (b) The series coefficients $a_{k}$ converge (c) The series coefficient $b_{k}$ converge. 
Figure 12: The parameters are chosen as $c=3$ and $g=1.1$. (a) Series solution for the heteroclinic orbit joining $x_{1}=-0.6382$ and $x_{2}=-0.8612$. Here $\phi^{+}(0)=\phi^{-}(0)=-0.4991$ (b) Converging series

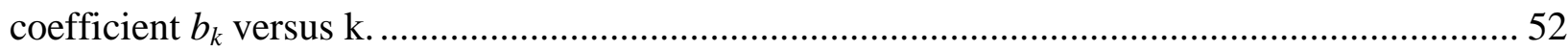

Figure 13: The parameters are chosen as $\mathrm{c}=-1$ and $\mathrm{g}=-1.25$. (a) Series solution for the homoclinic orbit to the point $x_{0}=0.4627$. (b) The series coefficients $a_{k}$ versus $\mathrm{k}$ does not converge. (c) The series coefficients $b_{k}$ versus $\mathrm{k}$ does not converge. 58 Figure 14: The parameters are chosen as $c=-1$ and $g=-1.25$. (a) Series solution for the homoclinic orbit to the point $x_{0}=0.4627$. Here, $a_{1}=0.04$ and $b_{1}=0.0348$ to have continuity a the origin: $\phi^{+}(0)=\phi^{-}(0)=0.4977$. (b) The series coefficients $a_{k}$ versus k converges. (c) The series coefficients $b_{k}$ versus k converges. 60 Figure 15: The parameters are chosen as $c=3$ and $g=0.1$ (a) Series solution for the homoclinic orbit to the point $x_{0}=-0.0370$. (b) The series coefficients $a_{k}$ versus k rapidly converges. (c) The series coefficients $b_{k}$ versus $\mathrm{k}$ rapidly converges. 61 Figure 16: The parameters are chosen as $c=1$ and $g=-1.25$. (a) Series solution for the homoclinic orbit to the point $x_{0}=-0.4627$. Here $a_{1}=0.04$ and $b_{1}=0.0521$ to have continuity a the origin $\phi^{+}(0)=\phi^{-}(0)=-0.4112$. (b) The series coefficients $a_{k}$ versus $\mathrm{k}$ converges. (c) The series coefficients $b_{k}$ versus k converges. 63 Figure 17: $\phi(x)$ plotted as a function of $x$ for $\mathrm{c}=0.5,1.0$, and 2.0 for (a) negative, and (c) positive values of $b_{1}$. The series coefficients $b_{k}$ verses $k$ is plotted for $\mathrm{c}=0.5,1.0$ and 2.0 for (b) negative, and (d) positive values of $b_{1}$. 
Figure 18: $\phi(x)$ plotted as a function of $\mathrm{x}$ for $\mathrm{c}=0.5$ at different time instances showing wave

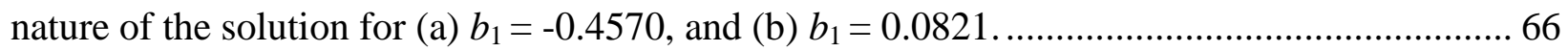
Figure 19: The parameters are chosen as $\mathrm{c}=2$ and $\mathrm{g}=0.8$, (a) Homoclinic orbit to the saddle point $z_{1}=(0.4436,0), x_{0}=0.4436$ (b) $a_{\mathrm{k}}$ versus $\mathrm{k}$, shows the series coefficients converge.......... 71 Figure 20: The parameters are chosen as $c=0.5$ and $g=0.13$, (a) Homoclinic orbit to the saddle point $z_{2}=0.3356$. Here $\phi(0)=0.44705$, (b) $a_{\mathrm{k}}$ versus $\mathrm{k}$, shows the series coefficients converges. 73

Figure 21: The parameters are chosen as $c=0.5$ and $g=-0.1$ (a) Homoclinic orbit to the saddle point $z_{2}=(-0.2218,0), x_{0}=-0.2218$, (b) $a_{\mathrm{k}}$ versus $\mathrm{k}$, shows the series coefficients converge. $\ldots . . .73$ Figure 22: (a) $\phi(x)$ plotted as a function of $x$ for different values of $t$ for $\mathrm{c}=0.5$ and $\mathrm{g}=0.014$ at the equilibrium point $z_{2}$, showing traveling wave nature of the solution Equation (3.17), here $a_{1}=$ 0.0357 (b) Plot of $a_{k}$ versus k shows the series coefficients are converging. 78

Figure 23: (a) $\phi(x)$ plotted as a function of $x$ for $t=0, \mathrm{c}=-1$ and $\mathrm{g}=0.06$, here $a_{1}=0.3066$ (b) Plot of $a_{\mathrm{k}}$ versus $k$ shows the series coefficients does not converge............................................ 78

Figure 24: (a) $\phi(x)$ plotted as a function of $x$ for different values of $t$ for $\mathrm{c}=0.5$ and $\mathrm{g}=0.014$ showing traveling wave nature of the solution Equation (3.17). 79

Figure 25: (a) $\phi(x)$ plotted as a function of $\mathrm{x}$ for $\mathrm{t}=0, \mathrm{c}=-1$ and $\mathrm{g}=0.06$, here $a_{1}=-0.0710$. (b) Plot of $a_{k}$ versus k shows the series coefficients converge. 79 


\section{CHAPTER 1: INTRODUCTION}

The Camassa-Holm (CH) equation [1],

$$
u_{t}+2 \kappa u_{x}-u_{x x t}+3 u u_{x}=2 u_{x} u_{x x}+u u_{x x x}
$$

where $u=u(x, t)$ and $\kappa$ is a constant (and subscripts denote partial derivatives), has attracted much research interest in recent years [2]. This equation came to prominence with the work of Camassa et al. in 1993 and 1994 [1], where it was argued that the equation could be taken as a model for the unidirectional propagation of waves in shallow water.

For $\kappa=0$, Camassa and Holm showed that Equation (1.1) has peakons of the form

$u(x, t)=c e^{-|x-c t|}$. In mathematics and physics, a soliton is a solitary wave packet or pulse that maintains its shape while traveling at constant speed. This type of wave has been the focus interest since solitons are thus stable, and do not disperse over time. Peakons are a type of non smooth soliton, which were discovered by Camassa and Holm in 1993; these waves have a sharp peak where it has a discontinuous derivative. The wave profile is similar to the graph of the function $e^{-|x|}$. 


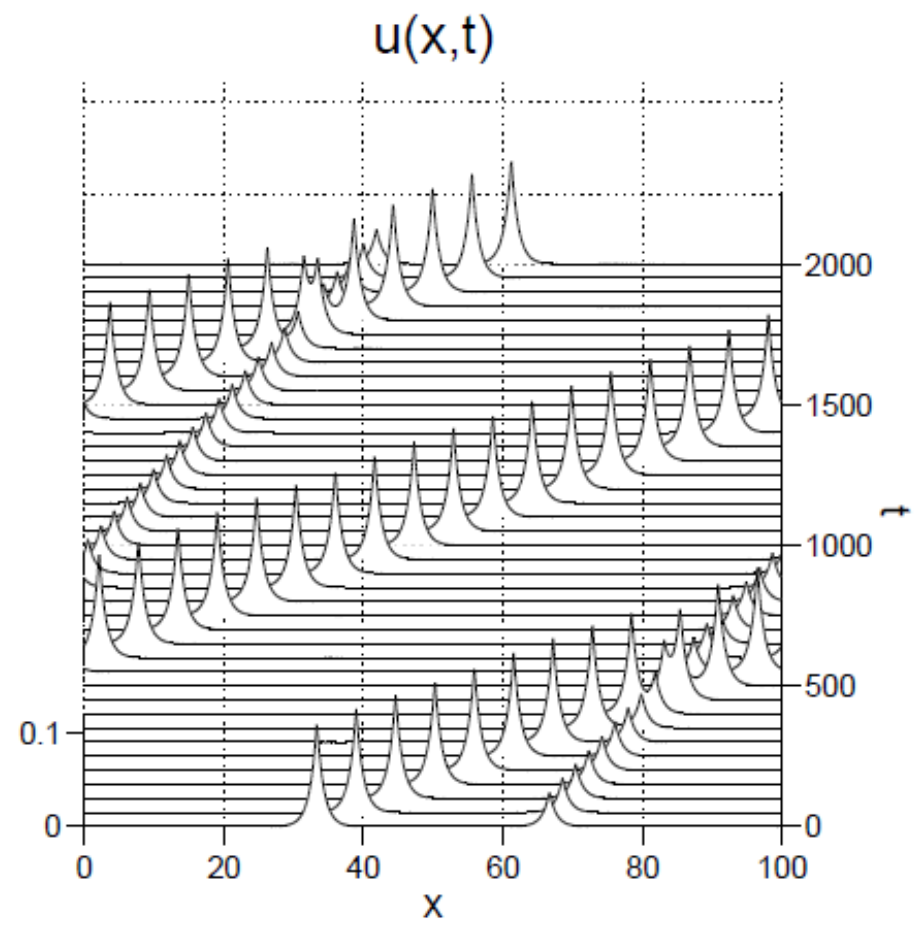

Figure 1: Plot of $u(\mathrm{x}, \mathrm{t})$ with the change of $x$ and $t$ showing two peakons with different wave speed colliding with each other forming double peakon (Image taken from Reference [3]).

Equation (1.1) can be re-written in the following form:

$$
\left(1-D_{x}^{2}\right) u_{t}=-2 \kappa u_{x}-3 u u_{x}+2 u_{x} u_{x x}+u u_{x x x}, \quad u=u(x, t), \quad D_{x}=\frac{\partial}{\partial x}
$$

belonging to the class:

$$
\left(1-D_{x}^{2}\right) u_{t}=F\left(u, u_{x}, u_{x x}, u_{x x x}, \ldots \ldots \ldots \ldots\right)
$$

which has attracted much interest, particularly the possible integrable member of equation $[3,4]$. The Camassa-Holm equation is integrable by the inverse scattering transform. It possesses an infinite hierarchy of local conservation laws, bi-Hamiltonian structure and the various other remarkable properties of integrable equations. Despite its non-evolutionary form, the CamassaHolm equation possesses an infinite hierarchy of local higher symmetries [4]. 
Until 2002, the Camassa-Holm equation was the only known integrable example of the type of Equation (1.3). Later, Degasperis and Procesi [3, and references therein] found another nonlinear PDE with similar properties, and this, so-called DP equation, has been studied quite intensively. Much more recently, Novikov [4] and Mikhailov and Novikov [5] showed that there are other different examples of Equation (1.3) which are integrable. Novikov presented a detailed summary of integrable and homogeneous polynomial generalizations of the Camassa-Holm type equation with quadratic and cubic nonlinearities [4]. He showed that the obtained equations can be treated as non-local symmetries of local scalar evolution quasi-linear integrable equations of orders 2,3 and 5.

In this thesis, the dynamical behavior of the traveling wave solutions of some of these generalized Camassa-Holm equations [4] is discussed. In particular, we consider the following four member nonlinear PDEs (NLPDEs):

$$
\begin{gathered}
\left(1-D_{x}^{2}\right) u_{t}=D_{x}\left(2-D_{x}\right)\left(1+D_{x}\right) u^{2} \\
\left(1-D_{x}^{2}\right) u_{t}=D_{x}\left(2+D_{x}\right)\left[\left(2-D_{x}\right) u\right]^{2} \\
\left(1-D_{x}^{2}\right) u_{t}=D_{x}\left(u^{2} u_{x x}-u_{x}^{2} u_{x x}+u u_{x}^{2}-u^{3}\right)
\end{gathered}
$$

and

$$
\left(1-D_{x}^{2}\right) u_{t}=D_{x}\left(4-D_{x}^{2}\right) u^{2}
$$

from Novikov's list of 27 generalized Camassa-Holm equations [4]. 
Since these integrable generalized $\mathrm{CH}$ equations are new, and the properties of the solutions of only one of them has been considered in any sort of depth [6], we investigate the possible travelling wave solutions of Equations (1.4)-(1.7) in detail in this thesis.

Two separate approaches are employed. Since Equations (1.4)-(1.7) are integrable generalized $\mathrm{CH}$ equations, non-smooth solutions as in the $\mathrm{CH}$ equation are a definite possibility. To investigate these, we employ a somewhat unusual variant of phase-plane analysis [7] which has been recently applied to consider peakon and cuspon solutions of a wide variety of NLPDEs. Since the approach is quite novel, we first review it in Chapter 2 before applying it to our PDEs in Chapter 3.

In Chapter 4, we consider regular smooth traveling wave solutions of our system of Equations (1.4)-(1.7). As is well known, homoclinic and heteroclinic orbits of the traveling wave ODE (of any PDE) correspond to pulse and front (shock or kink) solutions of the governing PDE.

In particular, we apply a recently developed technique [8,9] to analytically compute convergent multi-infinite series solutions for the possible homoclinic and heteroclinic orbits of the traveling-wave ODEs of Equations (1.4)-(1.7). They correspond to convergent series for pulse or front (shock) solutions of these generalized CH Equations (1.4) - (1.7). Since the later terms in the series fall off exponentially, we show high accuracy may be obtained for the pulse and front shapes using only small number of terms. The actual convergence of such series is analogous to the earlier treatments $[8,9]$, and is omitted here.

The remainder of this thesis is organized as follows. In Chapter 2, the recently developed theory for singular traveling-wave ODEs is reviewed [7]. This is then applied to our generalized 
CH systems Equations (1.4)-(1.7) in Chapter 3. Chapter 4 develops analytic pulse and front solutions of Equations (1.4)-(1.7). The results and the outlook for further work are summarized in Chapter 5. 


\section{CHAPTER 2: BASIC MATHEMATICAL THEORY OF SINGULAR TRAVELLING WAVE SYSTEMS}

\subsection{Some background on dynamical systems}

In this chapter, we begin by reviewing some background material on the phase plane analysis of dynamical systems. Virtually anything that evolves over time can be thought of as a dynamical system. A spring-mass system, a pendulum, economic growth, RLC circuits- these are

all examples of dynamical systems. A dynamical system has two parts: a state vector (consisting of the physical variables) which describes exactly the state of some real or hypothetical system, and a function (i.e., a rule) which tells us, given the current state, what will be the state of the system at the next instant of time.

A dynamical system consists of a phase (or state) vector $x \in R^{n}$. This vector can consist of several variables. For example, a ball tossed straight up can be described using two numbers: its height $h$ above the ground and its (upward) velocity $v$. Once we know these two numbers, $h$ and $v$, the time evolution of the ball is completely determined. The pair of numbers $(h, v)$ is a vector which completely describes the state of the ball and hence is called the state vector of the system. The second part of a dynamical system is a rule or a function, $f: R^{n} \rightarrow R^{n}$ 
Here the function $f(x)$ describes how the system evolves over time. We consider the following autonomous differential equation,

$$
\frac{d x}{d t}=f(x), \quad x \in R^{n}
$$

Assume that the function $f(x)$ satisfies Lipschitz condition for all $x$, and thus satisfies the condition of existence and uniqueness for the solution of Equation (2.1). The function given by $x \rightarrow(x, f(x))$ defines a vector field $\mathrm{V}$ on $\mathrm{R}^{\mathrm{n}}$ associated with Equation (2.1). A point $\left(x, x^{\prime}\right)$ on $\mathrm{R}^{2 \mathrm{n}}$ is called the Phase point or a state. We say that the evolution of $\boldsymbol{x}(t)$ is governed by the dynamical system.

For a differentiable function $f$, the derivative $D f$ is given by the $\mathrm{n} \times \mathrm{n}$ Jacobian matrix $D f=\left[\frac{\partial f_{i}}{\partial x_{j}}\right]$. A point $x_{0} \in \mathrm{R}^{\mathrm{n}}$ is called an equilibrium point or critical point of Equation (2.1) if $f\left(x_{0}\right)=0$. The linear system

$$
\frac{d x}{d t}=D f\left(x_{0}\right), \quad x \in R^{n}
$$

is called the linearized system of Equation (2.1) at the point $x_{0}$.

For an orbit $\varphi(t, x)$ of Equation (2.1) satisfying $\lim _{t \rightarrow \infty} \varphi(t, x)=\alpha$ and $\lim _{t \rightarrow-\infty} \varphi(t, x)=\beta, \varphi(t, x)$ is called a homoclinic orbit if $\alpha=\beta$, and $\varphi(t, x)$ is said to be a heteroclinic orbit if $\alpha \neq \beta$. Let $H: \mathrm{R}^{\mathrm{n}} \times \mathrm{R}^{\mathrm{n}} \rightarrow \mathrm{R}$ be a smooth function given by

$$
\left(q_{1}, q_{2}, \cdots, q_{n}, p_{1}, p_{2}, \cdots p_{n}\right) \rightarrow H\left(q_{1}, q_{2}, \cdots, q_{n}, p_{1}, p_{2}, \cdots p_{n}\right)
$$

and define the associated Hamiltonian system on $\mathrm{R}^{2 \mathrm{n}}$ with Hamiltonian $H$ by 


$$
\frac{d q_{i}}{d t}=\frac{\partial H}{\partial p_{i}}, \quad \frac{d p_{i}}{d t}=\frac{\partial H}{\partial q_{i}}, \quad i=1,2, \cdots n .
$$

Hamiltonian systems are conservative because the Hamiltonian function

$H\left(q_{1}, q_{2}, \cdots, q_{n}, p_{1}, p_{2}, \cdots p_{n}\right)$ remains constant along the orbit of Equation (2.3). We now consider an analytic planar system:

$$
\frac{d x}{d t}=P(x, y), \quad \frac{d y}{d t}=Q(x, y)
$$

which can be rewritten into the form of vector field $X=P(x, y) \frac{\partial}{\partial x}+Q(x, y) \frac{\partial}{\partial y}$. A system of Equation (2.4) is called integrable, if there exists a first integral $H(x, y)=h$, where $h$ denote an integral constant, such that

$$
\frac{d x}{d t}=P(x, y)=\mu(x, y) \frac{\partial H}{\partial y}, \quad \frac{d y}{d t}=Q(x, y)=-\mu(x, y) \frac{\partial H}{\partial x}
$$

The function $\mu(x, y)$ is called an integrating factor. In particular, if $\mu(x, y) \equiv 1$, then Equation (2.4) becomes a Hamiltonian system with one degree of freedom.

A critical point $\left(x_{0}, y_{0}\right)$ of a system of Equation (2.4) is a center if the real part of the eigenvalue of the Jacobian matrix evaluated at the critical point $\left(x_{0}, y_{0}\right)$, is zero. A critical point $\left(x_{0}, y_{0}\right)$ of a system of Equation (2.4) is a saddle point if the matrix 


$$
D f\left(x_{0}, y_{0}\right)=\left(\begin{array}{ll}
\frac{\partial P\left(x_{0}, y_{0}\right)}{\partial x} & \frac{\partial P\left(x_{0}, y_{0}\right)}{\partial y} \\
\frac{\partial Q\left(x_{0}, y_{0}\right)}{\partial x} & \frac{\partial Q\left(x_{0}, y_{0}\right)}{\partial y}
\end{array}\right)
$$

of the linearized system of Equation (2.4) at $\left(x_{0}, y_{0}\right)$ has one positive eigenvalue and one negative eigenvalue. A critical point of a system of Equation (2.4) is a node if $\operatorname{det}\left(D f\left(x_{0}, y_{0}\right)\right)>0$ and $\left(\operatorname{tr} D f\left(x_{0}, y_{0}\right)\right)^{2}-4 \operatorname{det}\left(D f\left(x_{0}, y_{0}\right)\right)>0$.

Having reviewed the basic background theory we next consider its application to traveling wave solutions of three generic classes of nonlinear PDEs [7] which cover many important systems.

\subsection{Phase portraits of traveling wave equations having singular straight lines}

\subsubsection{The dynamics of the first type of singular traveling waves}

We first consider the following three nonlinear evolution equations [7]

$$
\begin{array}{r}
u_{t t}=\left(g(u) u_{x}\right)_{x}+f(u) \\
u_{t t}=(f(u))_{x x}+\alpha^{2}(g(u))_{x x x x}
\end{array}
$$

and

$$
u_{t}=\left(G(u) u_{x}\right)_{x x}+(f(u))_{x}
$$

where $f, g$ and $G$ are smooth nonlinear functions, and $\alpha$ is a constant parameter. Substituting $u=\phi(x-c t)=\phi(\xi)$ into Equations (2.6)-(2.8), integrating twice and once for (2.7) and (2.8) respectively, we have the following three systems: 


$$
\begin{gathered}
\frac{d \phi}{d \xi}=y, \quad \frac{d y}{d \xi}=-\frac{g^{\prime}(\phi) y^{2}+f(\phi)}{g(\phi)-c^{2}}, \\
\frac{d \phi}{d \xi}=y, \quad \frac{d y}{d \xi}=-\frac{g^{\prime \prime}(\phi) y^{2}+\frac{1}{\alpha^{2}}\left(f(\phi)-c^{2} \phi\right)}{g^{\prime}(\phi)},
\end{gathered}
$$

and

$$
\frac{d \phi}{d \xi}=y, \quad \frac{d y}{d \xi}=-\frac{G^{\prime}(\phi) y^{2}+c \phi+f(\phi)}{G(\phi)},
$$

Clearly, these systems have the same form. So we can say that the above nonlinear wave models have traveling wave systems of the following general form:

$$
\frac{d \phi}{d \xi}=y, \quad \frac{d y}{d \xi}=-\frac{G^{\prime}(\phi) y^{2}+F(\phi)}{G(\phi)}
$$

System of Equation (2.12) also admits a first integral:

$$
H(\phi, y)=y^{2} G^{2}(\phi)+2 \int G(\phi) F(\phi) d \phi=h
$$

where, $h$ is the integral constant.

We assume $G, F$ are at least $C^{2}$ - functions in order to guarantee the existence and uniqueness of the solutions of the initial value problem.

(1) The function $G(\phi)$ has exactly one simple zero, at $\phi=\phi_{s}, G^{\prime}\left(\phi_{s}\right) \neq 0$;

(2) The function $F(\phi)$ has exactly two simple zeros, $\phi=\phi_{1,2^{\prime}}, \phi_{1}<\phi_{2}$ and $F^{\prime}\left(\phi_{1}\right)>0$, $F^{\prime}\left(\phi_{2}\right)<0$. 
Then, system of Equation (2.12) has two elementary critical points at $P_{1,2}\left(\phi_{1,2}, 0\right)$. In the straight line $\phi=\phi_{S}$ of the $(\phi, y)$ phase plane, the right hand of the second equation of (2.12) is discontinuous. We call $\phi=\phi_{S}$ a singular straight line in which there exist two critical points (2.12) at $S_{ \pm}\left(\phi_{s} \pm \sqrt{Y}\right)$ if $Y=-\frac{F\left(\phi_{s}\right)}{G^{\prime}\left(\phi_{s}\right)}>0$. We denote that $h_{1,2}=H\left(\phi_{1,2}, 0\right)$ and $h_{s}=$ $H\left(\phi_{S}, \pm \sqrt{Y}\right)$. For convenience, we will refer to the Equations (2.12) as "first type of singular traveling systems".

The so called "three step method" will be introduced into the following:

a) Make a transformation of the independent variable, such that the "singular system" (2.12) becomes a "regular system".

b) Discuss the dynamical behavior of the "regular system".

c) Use known dynamical behavior of the "regular system", to obtain the wave profiles determined by all the bounded solutions of the "singular system".

As the first step, let us make the transformation $d \xi=G(\phi) d \zeta$, for $\phi \neq \phi_{s}$ and the Equation (2.12) becomes:

$$
\frac{d \phi}{d \zeta}=G(\phi) y, \quad \frac{d y}{d \zeta}=-\left(G^{\prime}(\phi) y^{2}+F(\phi)\right)
$$

Obviously, the straight line $\phi=\phi_{s}$ now becomes an invariant straight line of Equation (2.14).

Since the systems of Equations in (2.12) and (2.14) have the same invariant curve solutions, as a second step, we study the associated "regular system" Equation (2.14) in order to get the phase portraits of Equation (2.12). In fact, for each orbit of Equation (2.14) with a given 
level curve of Equation (2.13), the essential difference between Equations (2.14) and (2.12) is the parametric representation of the orbit. Near $\phi=\phi_{s}$ Equation (2.14) uses the "fast time variable" $\zeta$, while Equation (2.12) uses the "slow time variable" $\xi$.

The Jacobian of the linearized system of Equation (2.14) at the critical point $P_{1,2}\left(\phi_{1,2}, 0\right)$ and $S_{ \pm}\left(\phi_{s}, \pm \sqrt{Y}\right)$ is given by

$$
J\left(\phi_{1,2}, 0\right)=F^{\prime}\left(\phi_{1,2}\right) G\left(\phi_{1,2}\right), \quad J\left(\phi_{s}, \pm \sqrt{Y}\right)=-2 Y\left(G^{\prime}\left(\phi_{s}\right)\right)^{2}
$$

By the theory of planar dynamics, we know that if $J<0$, then the critical point is a saddle point. Thus the critical points $S_{ \pm}$are saddle points.

If $\phi_{S} \neq \phi_{1,2}$, the critical point $\left(\phi_{1}, 0\right)$ is a saddle, when $G\left(\phi_{1}\right)>0$ or a center if $G\left(\phi_{1}\right)<$ 0 , while the critical point $\left(\phi_{2}, 0\right)$ is a center (or a saddle point).

If $\phi_{S}=\phi_{1,2}$, the critical point $\left(\phi_{1}, 0\right)$ or $\left(\phi_{2}, 0\right)$ is a second - order critical point.

\subsubsection{Main theorems to identify the profiles of waves}

In this section, we will review three theorems [7] in order to identify the profiles of traveling wave solutions of Equations (2.6)-(2.8) determined by different phase orbits of Equation (2.14). The traveling wave system having least singular straight line is shown. The essential difference of profiles of nonlinear waves determined by system of Equations (2.12) and (2.14) is also pointed out here.

For a planar dynamical system of Equation (2.14), by the theory of invariant manifolds, all periodic solutions and all stable and unstable manifolds connecting critical points have the 
same smoothness as the given vector field with respect to time variable $\zeta$. But for system of Equation (2.12), even though it has the same invariant level curves as system of Equation (2.14), the smooth property of orbits of Equation (2.12) with respect to time variable $\xi$ should be studied, because the line $\phi=\phi_{S}$ is not an orbit of Equation (2.12). In order to understand the dynamical behavior of Equation (2.12) with respect to the time variable $\xi$, the behavior of the orbits closer to the singular straight line $\phi=\phi_{S}$ needs to be studied, i.e. we should be able to distinguish the singular straight line $\phi=\phi_{S}$ of Equation (2.12) geometrically from the straight line solution $\phi=\phi_{S}$ of Equation (2.14).

We consider two types of phase portraits of Equation (2.14), which are drawn in Figure 2. In Figure 2 on the left-hand side of the straight line $\phi=\phi_{s}=0$ there is a periodic annulus around the center $P_{2}\left(\phi_{2}, 0\right)$, which has a boundary curves consisting of segment $S_{1} S_{2}$ and an arc $\widehat{S_{1} S_{2}}$ defined by the level curve $H(\phi, y)=h_{s}$ as given in the Equation (2.13). If $\zeta \rightarrow \pm \infty$, along these two orbits the phase point $(\phi(\zeta), y(\zeta))$ of Equation (2.14) tend to the critical points $S_{1}$ and $S_{2}$. But for the Equation (2.12), $S_{1} S_{2}$ lies on the singular straight line $\phi=\phi_{S}$. An orbit $\gamma$ of the family of periodic orbits in the periodic annulus of $P_{2}$ is a closed branch of the invariant curves $H(\phi, y)=h_{\gamma}$, where $h_{\gamma} \in\left(H\left(\phi_{2}, 0\right), h_{s}\right)$.

We can see in Figure 2 that if a point $\mathrm{C}$ near the left boundary curve, starts moving along the periodic orbit $\gamma$ whose energy level $h_{\gamma}$ is close to $h_{s}$, then as $\xi$ increases the point will move to the right until it reaches the turning point $A_{\gamma}$, at the intersection point with the horizontal inclination curve $\Gamma_{h}: G^{\prime}(\phi) y^{2}+F(\phi)=0$ of the vector fields defined by the Equation (2.12). Then, it will fall down almost vertically, to the lower arc of $\gamma$. 

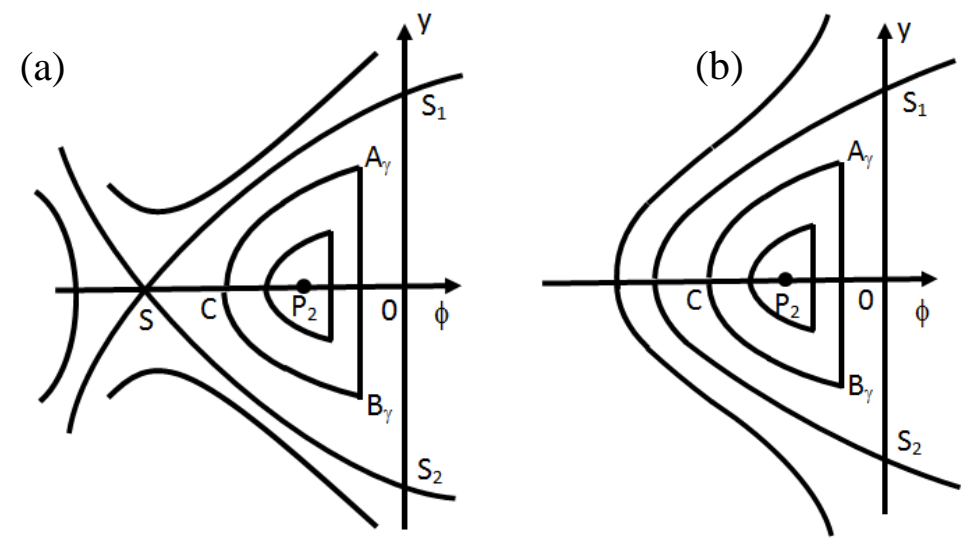

Figure 2: Two types of phase portraits of Eq. (2.14) [7].

After passing through another turning point $B_{\gamma}$, it will move to the left, and complete its motion of one period to C. Suppose that $B_{\gamma \varepsilon}$ and $A_{\gamma \varepsilon}$ are two points in the right neighborhood of $B_{\gamma}$ and $A_{\gamma}$ respectively, on the orbit of $\gamma$.

Theorem 1: (The rapid jump property of $\frac{d \phi}{d \xi}=y$ near the singular straight line) When $h \rightarrow h_{s}$, the periodic orbits of the periodic annulus surrounding $P_{2}$ approach the boundary curves. Let $\left(\phi, \frac{d \phi}{d \xi}=y\right)$ be a point on the periodic orbit $\gamma$ of Equation (2.12). Then, along the line segment $A_{\gamma \varepsilon} B_{\gamma \varepsilon}$ near the straight line $\phi=\phi_{s}$, in a very short time interval of $\xi, \frac{d \phi}{d \xi}=y$ jumps down rapidly[7].

Proof: The first conclusion follows from the continuity of the level set $H(\phi, y)=h$ given by Equation (2.13). We will show the second conclusion. Near the segment $\phi=\phi_{s}$, we assume that $\phi_{S}-\phi=\varepsilon \ll 1$. Notice that $\phi_{S}$ is a simple zero of $G(\phi)$. 

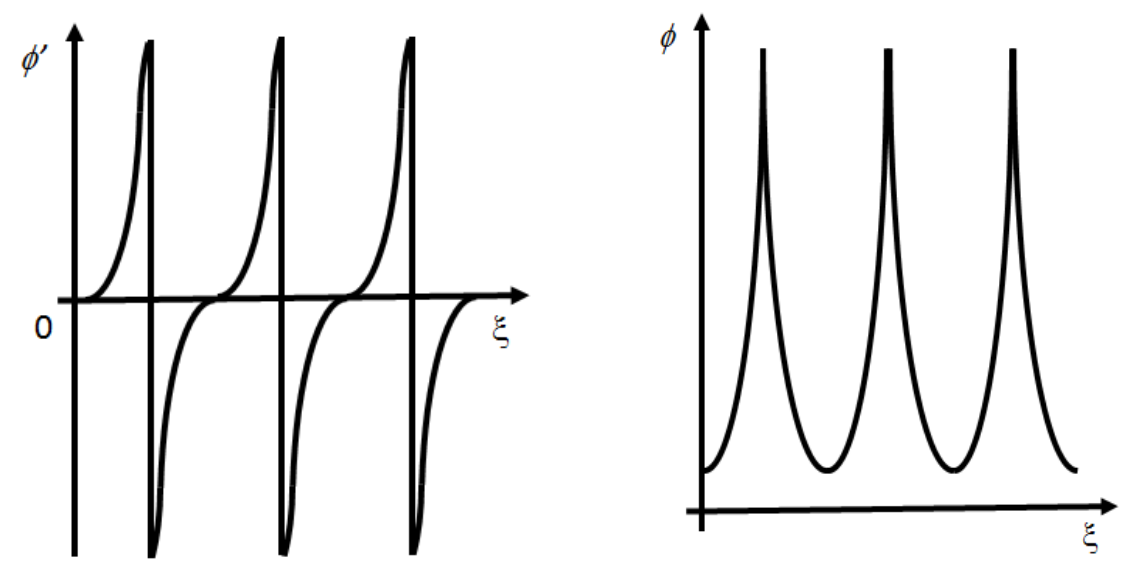

Figure 3: $\phi(\xi)$ and $\phi^{\prime}(\xi)$ plotted against $\xi$ corresponding to Figure 2.1 [7].

Thus, we can write the system of Equation (2.12) as the following relaxation oscillation "slow system" with the "slow time scale" $\xi$.

$$
\frac{d \phi}{d \xi}=y, \quad \varepsilon \frac{d y}{d \xi}=-\left(y^{2}-Y-O(\varepsilon)\right),
$$

where, $Y=-\frac{F\left(\phi_{s}\right)}{G^{\prime}\left(\phi_{S}\right)} \neq 0$

We have from Equation (2.15) that

$$
\frac{d \phi}{d y}\left(-y^{2}+Y+O(\varepsilon)\right)=\varepsilon y,
$$

Thus, as $\varepsilon \rightarrow 0$, the segment $B_{\gamma \varepsilon} A_{\gamma \varepsilon}$ of every periodic orbit will tend to the segment $S_{1} S_{2}$ in the straight line $\phi=\phi_{S}$. 
Now we consider the periodic orbit $\gamma$ near the energy level $h=h_{s}$ (Figure 2). According to the Equation (2.15), the period of $\gamma$ satisfies

$$
T=\oint_{\gamma} \frac{d \phi}{y}=\left(\int_{B_{\gamma \varepsilon} A_{\gamma \varepsilon}}+\int_{A_{\gamma \varepsilon} C B_{\gamma \epsilon}}\right) \frac{d \phi}{y}=T_{1}+T_{2}
$$

The contribution over the straight segment $y \in B_{\gamma \varepsilon} A_{\gamma \varepsilon}$ is

$$
T_{1}=\int_{y_{B_{\gamma \varepsilon}}}^{y_{A_{\gamma \varepsilon}}} \frac{d \phi}{y}=\int_{y_{B_{\gamma \varepsilon}}}^{y_{A_{\gamma \varepsilon}}} \frac{d \phi d y}{y d y}=\int_{y_{B_{\gamma \varepsilon}}}^{y_{A_{\gamma \varepsilon}}} \frac{\varepsilon d y}{-y^{2}+Y+O(\varepsilon)}=O(\varepsilon)
$$

Because $y^{2}-Y-O(\varepsilon) \neq 0$ along the straight segment from $B_{\gamma \varepsilon}$ to $A_{\gamma \varepsilon}$. Thus, Equation (2.18) implies that in a very short time interval of $\xi, \phi^{\prime}(\xi)$ jump up rapidly as in Figure 2(a). Figure 3 shows the profiles of $\phi^{\prime}(\xi)$ and $\phi(\xi)$ with respect to the variable $\xi$.

Theorem 2: (Existence of the finite time intervals of solutions with respect to $\xi$ in the positive or negative directions) Let $\left(\phi, \frac{d \phi}{d \xi}=y\right)$ be the parametric representation of an orbit $\gamma$ of system of Equation (2.12) and $\left(\phi_{S}, \pm \sqrt{Y}\right)$ be two points on the singular straight line $\phi=\phi_{S}$. Suppose that one of the following three conditions holds:

a) $Y>0$ and, along the orbit $\gamma$, as $\xi$ increases or decreases, the phase point $(\phi(\xi), y(\xi))$ tends to the points $\left(\phi_{s} \pm \sqrt{Y}\right)$, respectively.

b) $Y=0$ and, along the orbit $\gamma$, as $\xi$ increases or decreases, the phase point $(\phi(\xi), y(\xi))$ tends to the point $\left(\phi_{s}, 0\right)$ and $\gamma$ is in contact with the $y$-axis at the point $\left(\phi_{s}, 0\right)$. 

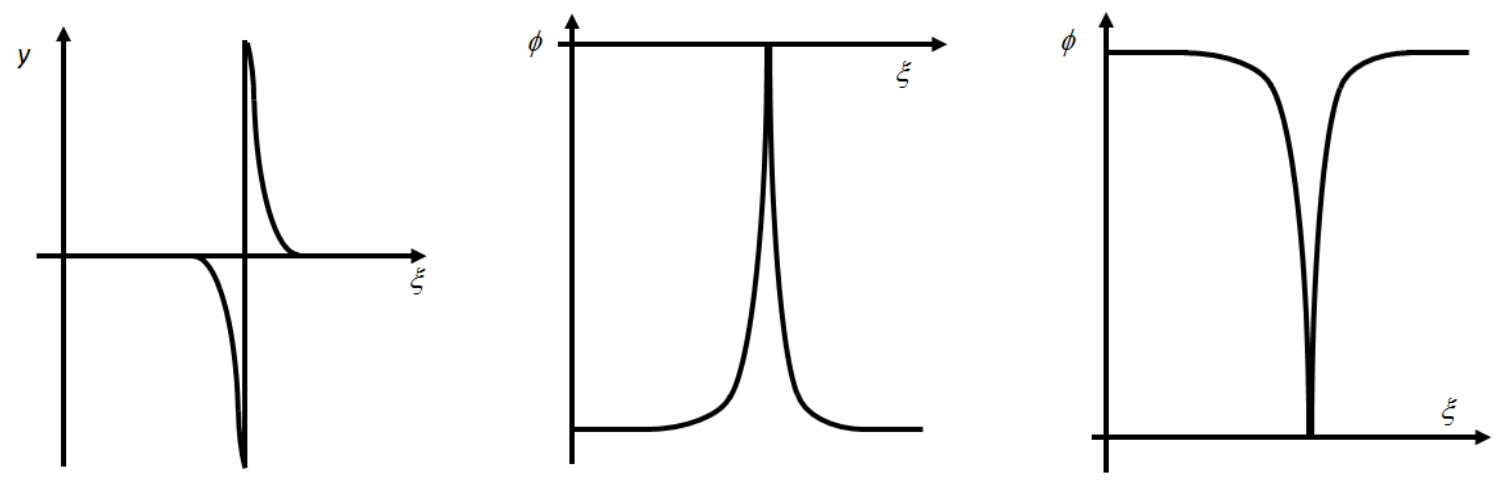

Figure 4: The profiles of $y=\phi^{\prime}(\xi)$ and solitary waves of two types [7].

c) Along the orbit $\gamma$, as $\xi$ increases or decrease, the phase point $(\phi(\xi), y(\xi))$ approaches the straight line $\phi=\phi_{S}$ in the positive direction or negative direction respectively, and $\lim _{\phi \rightarrow \phi_{s}}|y|=\infty$.

Then, there exist a finite value $\xi=\tilde{\xi}$ such that $\lim _{\xi \rightarrow \tilde{\xi}} \phi(\xi)=\phi_{S}$.

\section{Theorem 3:}

1) The curve triangle in Figure 2(a) defined by $H(\phi, y)=h_{s}$ gives rise to a solitary cusp wave solutions of the peak type, called peakon, of Equation (2.6)-(2.8) as in Figure 4.

2) The arch curves in Figure 2(b) defined by $H(\phi, y)=h_{s}$ gives rise to a periodic cusp wave of the peak type, called Cuspons, of Equation (2.6)-(2.8).

3) The oval curve defined by $H(\phi, y)=h_{s}$ gives rise to a smooth periodic wave solutions, called Compacton, of Equations (2.6)-(2.8).

4) The family of open curve (which approaches the singular straight line $\phi=\phi_{s}$ in two direction of $\xi$ and is defined by $(\phi, y)=h, h \in\left(h_{i}, h_{s}\right)$, where $\left(\phi_{i}, 0\right)$ is a saddle point) 
gives rise to uncountably infinitely many bounded breaking wave solutions of $\phi(\xi)$ of Equations (2.6)-(2.8).

5) The stable and unstable manifolds of a saddle point gives rise to a one-sided breaking kink wave solutions and an one-sided breaking anti-kink wave solutions of Equations (2.6)-(2.8) respectively.

\subsubsection{The dynamics of the second type of singular traveling waves}

The second class of singular traveling wave system is given by [10]

$$
\frac{d \phi}{d \xi}=y, \quad \frac{d y}{d \xi}=\frac{Q(\phi, y)}{f(\phi, y)}
$$

where the function $\mathrm{f}(\phi, \mathrm{y})$ and $\mathrm{Q}(\phi, \mathrm{y})$ satisfies $\mathrm{y} \frac{\partial \mathrm{f}(\phi, \mathrm{y})}{\partial \phi}+\frac{\partial \mathrm{Q}(\phi, \mathrm{y})}{\partial \mathrm{y}} \equiv 0$, which implies there exists a first integral of Equation (2.19). It is also notice that $\frac{d y}{d \xi}=\frac{Q(\phi, y)}{f(\phi, y)}$ is not defined on the set of real planar curves $f(\phi, y)=0$, and when the phase point $(\phi, y)$ passes through every branch of $f(\phi, y)=0, \frac{d y}{d \xi}$ changes sign [10]. We also consider the following system, which is called the associated regular system of Equation (2.19)

$$
\frac{d \phi}{d \zeta}=y f(\phi, y), \quad \frac{d y}{d \zeta}=Q(\phi, y)
$$

where $\mathrm{d} \xi=\mathrm{f}(\phi, \mathrm{y}) \mathrm{d} \zeta$, for $\mathrm{f}(\phi, \mathrm{y}) \neq 0$.

For the second type of singular traveling system of Equation (2.19), the existence of the singular curve $f(\phi, y)=0$ implies that there exists a breaking wave solution $\phi(\xi)$ of the corresponding nonlinear wave equation, on the singular curve $f(\phi, y)=0$, even though the 
associated regular system of Equation (2.20) has a family of smooth periodic solutions and homoclinic or heteroclinic orbits.

Having laid out the basic background theory of singular traveling waves, we will attempt to apply it next to our new family of generalized Camassa- Holm equations (1.4)-(1.7). 


\section{CHAPTER 3: PHASE PLANE ANALYSIS OF THE GENERALIZED CAMASSA-HOLM EQUATIONS: POSSIBLE SINGULAR AND REGULAR SOLUTIONS}

Having outlined the basic theory and technique for analyzing non-smooth traveling wave solutions of NLPDEs in Chapter 2, we now apply it to our generalized Camassa-Holm Equations (1.4)-(1.7). These equations are considered in turn in Sections 3.1 - 3.4.

\subsection{Phase portrait/possible solutions of Equation (1.4)}

Equation (1.4) can be simplified and written as

$$
u_{t}-u_{x x t}=\frac{\partial}{\partial x}\left[2 u^{2}+2 u u_{x}-2 u u_{x x}-2 u_{x}^{2}\right]
$$

Now, let $u(x, t)=\phi(x-c t)=\phi(z)$, where $z=x-c t$ and $c$ is the wave speed. Then we have the following

$$
u_{t}=-c \frac{d \phi}{d z}, \quad u_{x}=\frac{d \phi}{d z}, \quad u_{x x}=\frac{d^{2} \phi}{d z^{2}}, \quad u_{x x t}=-c \frac{d^{3} \phi}{d z^{3}}
$$

Then equation (3.1) becomes,

$$
-c \frac{d \phi}{d z}+c \frac{d^{3} \phi}{d z^{3}}=\frac{d}{d z}\left[2 \phi^{2}-2 \phi \frac{d^{2} \phi}{d z^{2}}-2\left(\frac{d \phi}{d z}\right)^{2}+2 \phi \frac{d \phi}{d z}\right]
$$


Integrating this equation once with respect to $\mathrm{z}$,

$$
-c \phi+c \frac{d^{2} \phi}{d z^{2}}=\left[2 \phi^{2}-2 \phi \frac{d^{2} \phi}{d z^{2}}-2\left(\frac{d \phi}{d z}\right)^{2}+2 \phi \frac{d \phi}{d z}+g\right]
$$

where $g$ is the constant of integration.

Equation (3.2) is equivalent to the following 2-dimensional systems

$$
\frac{d \phi}{d z}=y, \quad \frac{d y}{d z}=\frac{2 \phi^{2}-2 y^{2}+c \phi+2 \phi y+g}{c+2 \phi}
$$

which is the traveling wave system for Equation (1.4)

The system of Equation (3.3) has the two regular equilibria, $\mathrm{z}_{1}=\left(\frac{-\mathrm{c}+\sqrt{\mathrm{c}^{2}-8 \mathrm{~g}}}{4}, 0\right)$ and $\mathrm{z}_{2}=\left(\frac{-\mathrm{c}-\sqrt{\mathrm{c}^{2}-8 \mathrm{~g}}}{4}, 0\right)$, when $\mathrm{g} \leq \frac{\mathrm{c}^{2}}{8}$. Notice that when $\mathrm{g}=0$ the point $\mathrm{z}_{1}$ reduces to the origin and when $g=\frac{c^{2}}{8}$ the two equilibria collapse into the unique equilibrium $\mathrm{z}=\left(-\frac{\mathrm{c}}{4}, 0\right)$. In the straight line $\phi=-\frac{c}{2}$, of the $(\phi, y)$ phase plane, the second equation of (3.3) is discontinuous. The straight line $\phi=\phi_{\mathrm{s}}=-\frac{\mathrm{c}}{2}$ is the singular straight line described in the previous chapter.

As described in chapter 2 , to avoid this discontinuity on the singular straight line we make the transformation $\mathrm{dz}=(\mathrm{c}+2 \phi) \mathrm{d} \zeta$. Under this transformation Equation (3.3) becomes,

$$
\frac{d \phi}{d \zeta}=y(c+2 \phi), \quad \frac{d y}{d \zeta}=2 \phi^{2}-2 y^{2}+c \phi+2 \phi y+g
$$

System of Equation (3.4) is the associated regular system, of Equation (3.3). Since the first integral of both Equations (3.3) and (3.4) are same, therefore both of them have the same phase 
orbit, except on the straight line $\phi=\phi_{\mathrm{s}}=-\frac{\mathrm{c}}{2}$. The equilibrium points on the singular straight line are $z_{3}=\left(\frac{-c}{2}, \frac{-c+\sqrt{c^{2}+8 g}}{4}\right)$ and $z_{4}=\left(\frac{-c}{2}, \frac{-c-\sqrt{c^{2}+8 g}}{4}\right)$.

We will study the stability analysis of the equilibrium points $\mathrm{z}_{1}, \mathrm{z}_{2}, \mathrm{z}_{3}$ and $\mathrm{z}_{4}$ using the linearized system of Equation (3.4).

For $\mathrm{c}>0$ and $0<g<\frac{\mathrm{c}^{2}}{8}$, the equilibrium points $\mathrm{z}_{1}, \mathrm{z}_{3}$ and $\mathrm{z}_{4}$ are saddle point, while the equilibrium point $\mathrm{z}_{2}$ is a stable node.

For $\mathrm{c}>0, \mathrm{~g}<0$ and $|\mathrm{g}|<\frac{\mathrm{c}^{2}}{8}$, there is a change in the dynamical behavior of the equilibrium points $\mathrm{z}_{2}$ and $\mathrm{z}_{3}$. Now the equilibrium points $\mathrm{z}_{1}, \mathrm{z}_{2}$ and $\mathrm{z}_{4}$ are saddle point, while the equilibrium point $\mathrm{z}_{3}$ is a stable node.

For $\mathrm{c}<0$ and $0<g<\frac{\mathrm{c}^{2}}{8}$, the equilibrium point $\mathrm{z}_{1}$ is an unstable node while $\mathrm{z}_{2}, \mathrm{z}_{3}$ and $\mathrm{z}_{4}$ are saddle point.

For $\mathrm{c}<0, \mathrm{~g}<0$ and $|\mathrm{g}|<\frac{\mathrm{c}^{2}}{8}$, the equilibrium point $\mathrm{z}_{1}, \mathrm{z}_{2}$ and $\mathrm{z}_{3}$ are saddle points, while $\mathrm{z}_{4}$ is an unstable node.

When the constant of integration ' $\mathrm{g}$ ' is equal to zero, the equilibrium points of Equation (3.4) are $\mathrm{z}_{1}=(0,0), \mathrm{z}_{2}=\left(-\frac{\mathrm{c}}{2}, 0\right)$ and $\mathrm{z}_{3}=\left(\frac{-\mathrm{c}}{2}, \frac{-\mathrm{c}}{2}\right)$. Here $\mathrm{z}_{1}$ and $\mathrm{z}_{2}$ are the regular fixed points, while $\mathrm{z}_{3}$ is a singular fixed point.

The equilibrium point $\mathrm{z}_{1}$ is a saddle point for both $\mathrm{c}>0$ and $\mathrm{c}<0$. 
Let $\mathrm{M}\left(\mathrm{z}_{2}\right)$ be the coefficient matrix of the linearized system of Equation (3.4) evaluated at the critical point $\mathrm{z}_{2}=\left(\frac{-\mathrm{c}}{2}, 0\right)$. The determinant of the Jacobian matrix evaluated at $\mathrm{z}_{2}$ is zero. Thus, we cannot conclude anything about the stability of the equilibrium point $z_{2}$, from the linearized system of Equation (3.4).

Similarly the singular equilibrium point $\mathrm{z}_{3}=\left(\frac{-\mathrm{c}}{2}, \frac{-\mathrm{c}}{2}\right)$ is a saddle point for both $\mathrm{c}>0$ and $\mathrm{c}<0$.

Depending on the parameter (g, c) the phase portraits of Equation (3.4) are drawn in Figure 5. In general, besides the heteroclinic orbit in Figure 5(a) and 5(c) (which will be computed in chapter 4), the other significant feature is that the stable manifolds of the saddles act as separatrices dividing the phase-plane into disjoint regions.

We can see from Figure 5 that there are no closed orbits adjacent to the singular straight line $\phi=\phi_{\mathrm{S}}=-\frac{\mathrm{c}}{2}$ which, as they limit to the singular straight line $\phi=\phi_{\mathrm{S}}=-\frac{\mathrm{c}}{2}$, could give us singular solutions. And from the discussion and theorems of the previous chapter, in the absence of either closed arched curves or curved triangles, neither singular peakons nor cuspons are possible. 

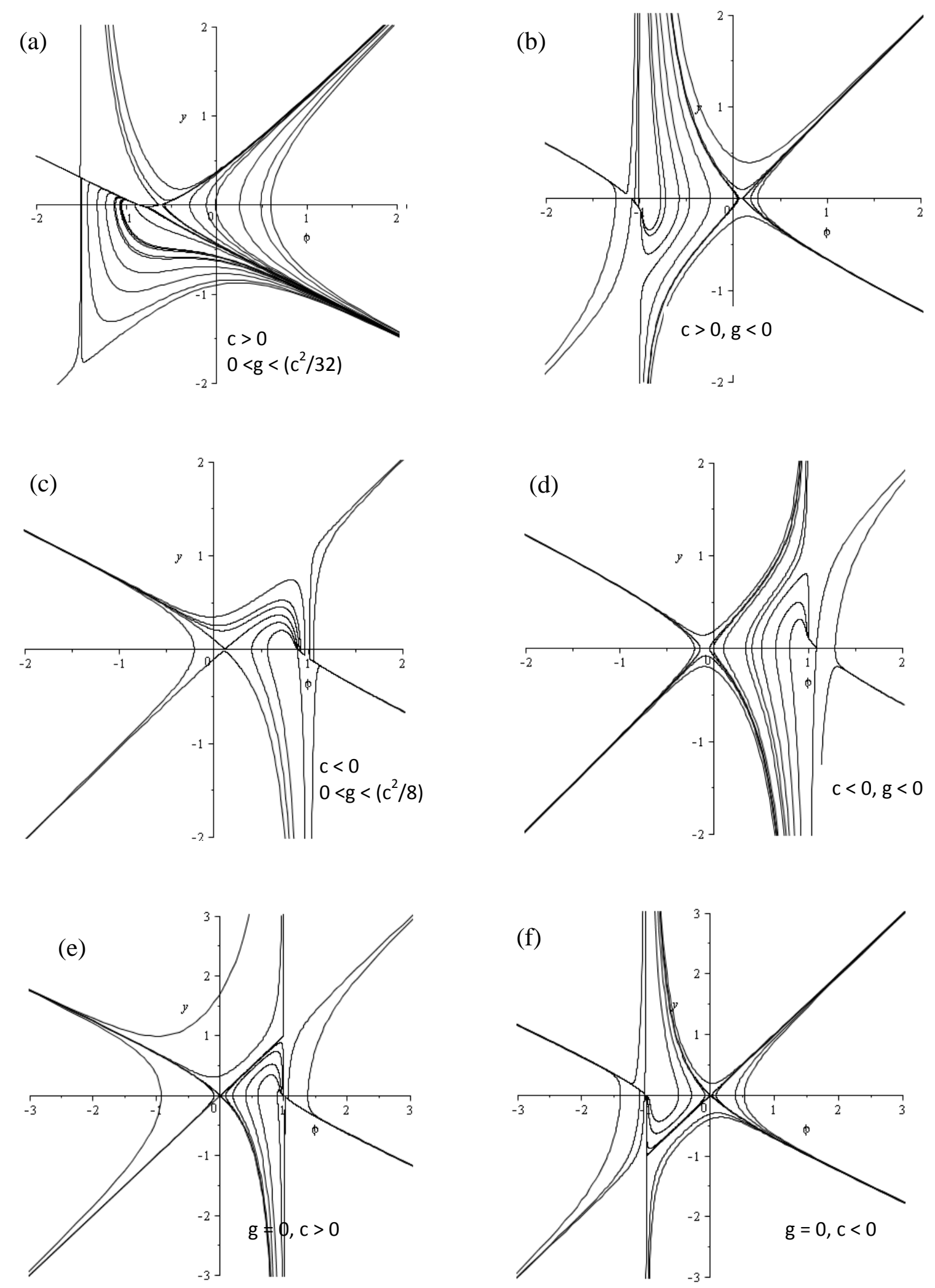

Figure 5: The phase portraits (y plotted against $\phi$ ) of Equation (3.4). 


\subsection{Phase portrait/possible solutions of Equation (1.5)}

Now we will discuss the phase portrait of Equation (1.5). Equation (1.5) can be written as

$$
u_{t}-u_{x x t}=\frac{\partial}{\partial x}\left(8 u^{2}-4 u u_{x x}-2 u_{x}^{2}+2 u_{x} u_{x x}\right)
$$

Now, let $u(x, t)=\phi(x-c t)=\phi(z)$, where $z=x-c t$ and $c$ is the wave speed.

As before we obtain,

$$
-c \phi+c \frac{d^{2} \phi}{d z^{2}}=8 \phi^{2}-4 \phi \frac{d^{2} \phi}{d z^{2}}-2\left(\frac{d \phi}{d z}\right)^{2}+2 \frac{d \phi}{d z} \frac{d^{2} \phi}{d z^{2}}+g
$$

where $\mathrm{g}$ is the constant of integration.

Equation (3.6) is equivalent to the following 2- dimensional system

$$
\frac{d \phi}{d z}=y, \quad \frac{d y}{d z}=\frac{8 \phi^{2}-2 y^{2}+c \phi+g}{c+4 \phi-2 y}
$$

Equation (3.7) is a second type of singular traveling wave system for the equation (1.5), which has the general form of equation (2.19). Here $c+4 \phi-2 y=0$ defines the set of real planar curves, such that second part of equation (3.7) is discontinuous on these curves, and when the phase point $(\phi, y)$ passes through every branch of $c+4 \phi-2 y=0, \frac{d y}{d z}$ changes sign.

Let us make a transformation $d z=(c+4 \phi-2 y) d \zeta$, for $c+4 \phi-2 y \neq 0$, then equation (3.7) becomes, 


$$
\frac{d \phi}{d \zeta}=y(c+4 \phi-2 y), \quad \frac{d y}{d \zeta}=8 \phi^{2}-2 y^{2}+c \phi+g
$$

Equation (3.8) is the associated regular system of (3.7).

Let, $L(\phi)=8 \phi^{2}+c \phi+g$ and $L^{\prime}(\phi)=16 \phi+c$. We can show that for a fixed $c>0$ the following hold.

1) $g>0$. When $g>\frac{c^{2}}{32}, L(\phi)$ has no real zero; when $g=\frac{c^{2}}{32}, L(\phi)$ has a double zero $z_{12}=$ $-c / 16$; when $g<\frac{c^{2}}{32}$ then $L(\phi)$ has two simple zeros $z_{1}<-\frac{c}{16}<z_{2}$.

2) $g<0$. When $|g|>\frac{c^{2}}{32}, L(\phi)$ has two simple zeros $z_{1}<0<z_{2}$; when $|g|=\frac{c^{2}}{32}$, then $L(\phi)$ has two simple zeros $z_{1}<-g<0<z_{2}$; when $|g|<\frac{c^{2}}{32}, L(\phi)$ has two simple zeros $z_{1}<-g<0<z_{2}$

The equilibrium points of Equation (3.8) are, $z_{1}=\left(\frac{-c+\sqrt{c^{2}-32 g}}{16}, 0\right)$ $z_{2}=\left(\frac{-c-\sqrt{c^{2}-32 g}}{16}, 0\right)$ and $z_{3}=\left(\frac{-c^{2}+2 g}{6 c}, \frac{c^{2}+4 g}{6 c}\right)$. Here $z_{1}$ and $z_{2}$ are the regular equilibrium point, while $z_{3}$ is a singular equilibrium point. We use the linearized system of Equation (3.8) to study the stability of these equilibrium points.

For $g>0, c>0$ and $c^{2}-32 g>0$, the equilibrium point $z_{1}$ and $z_{3}$ are saddle, while the equilibrium point $z_{2}$ is a center. The phase portrait is drawn in Figure 6(a). 
For $g>0, \mathrm{c}>0$ and $g=\frac{c^{2}}{32}$, the equilibrium point $z_{1}=z_{2}=\left(\frac{-c}{16}, 0\right)$ is a center, while the equilibrium point $z_{3}=\left(\frac{-c^{2}+2 g}{6 c}, \frac{c^{2}+4 g}{6 c}\right)$ is a saddle. The phase portrait is drawn in Figure 6(b).

For $g<0$, c $>0$ and $g>-\frac{c^{2}}{32}$, the equilibrium point $z_{1}$ and $z_{3}$ are saddle, while the equilibrium point $z_{2}$ is a center. The phase portrait is drawn in Figure 6(c).

For $g>0, \mathrm{c}<0$ and $g<\frac{c^{2}}{32}$, the equilibrium point $z_{2}$ and $z_{3}$ are saddle, while the equilibrium point $z_{1}$ is a center. The phase portrait is drawn in Figure $6(\mathrm{~d})$.

For $g<0$, c $<0$, and $g>-\frac{c^{2}}{32}$, the equilibrium point the equilibrium point $z_{2}$ and $z_{3}$ are saddle, while the equilibrium point $z_{1}$ is a center. The phase portrait is drawn in Figure 6(e).

For $g=0$, Equation (3.8) has the equilibrium points, $\mathrm{z}_{1}=(0,0), \mathrm{z}_{2}=\left(-\frac{\mathrm{c}}{8}, 0\right)$ and $\mathrm{z}_{3}=\left(\frac{-\mathrm{c}}{6}, \frac{-\mathrm{c}}{6}\right)$, where $\mathrm{z}_{1}$ and $\mathrm{z}_{2}$ are the regular equilibrium points while $\mathrm{z}_{3}$ is the singular equilibrium point, of these $\mathrm{z}_{1}=(0,0)$ and $\mathrm{z}_{3}=\left(\frac{-\mathrm{c}}{6}, \frac{-\mathrm{c}}{6}\right)$ are saddle points, where as $\mathrm{z}_{2}=$ $\left(-\frac{c}{8}, 0\right)$ is a center. The phase portraits of Equation (3.8) for $g=0$ are drawn in Figure $6(f)$ and Figure $6(\mathrm{~g})$ for $\mathrm{c}>0$ and $\mathrm{c}<0$ respectively.

We see that there exists a homoclinic orbit at the origin for $g=0$ [Figure $6(\mathrm{f}), 6(\mathrm{~g})]$, whose series solution will be calculated in Chapter 4. We will also compute the homoclinic orbit for $g \neq 0$. 


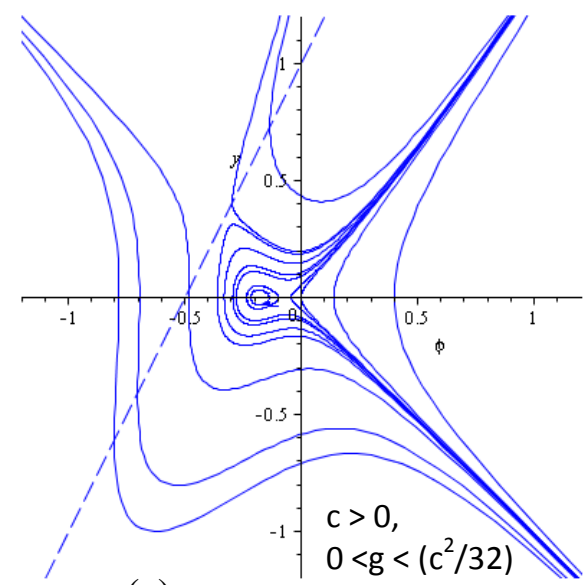

(a)
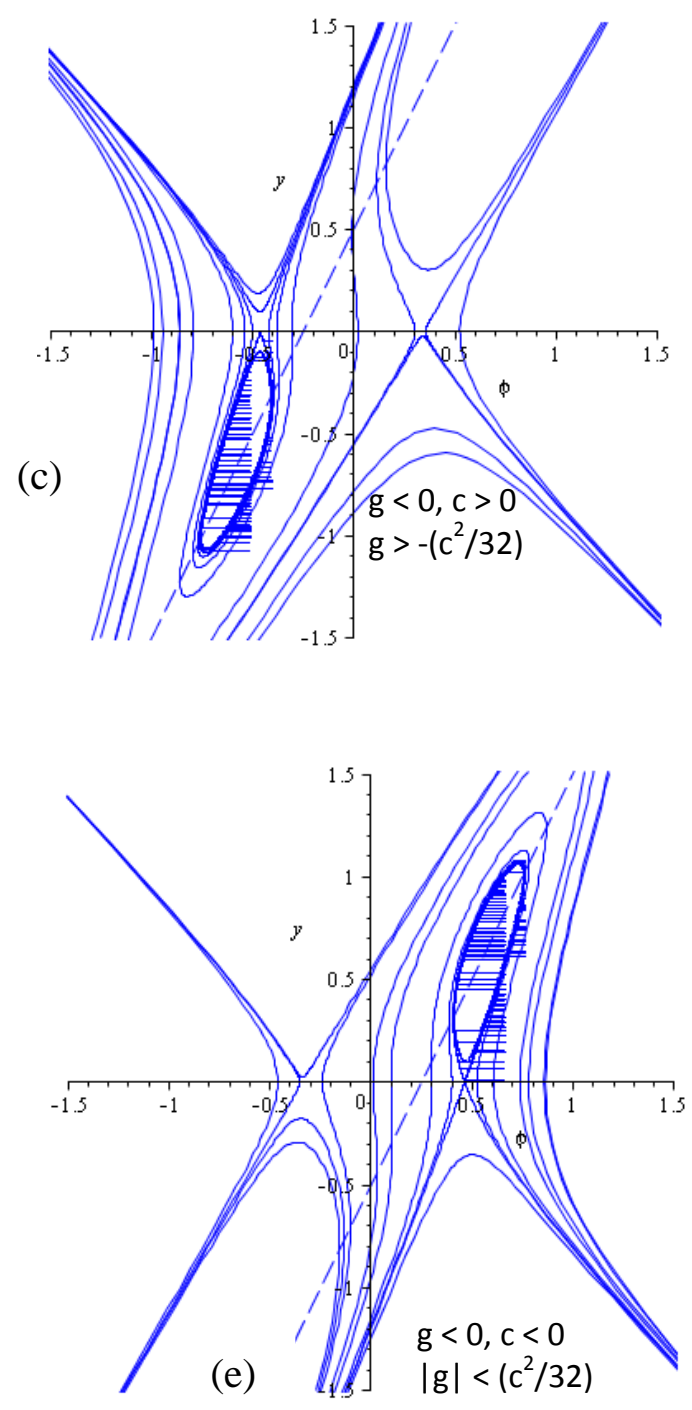
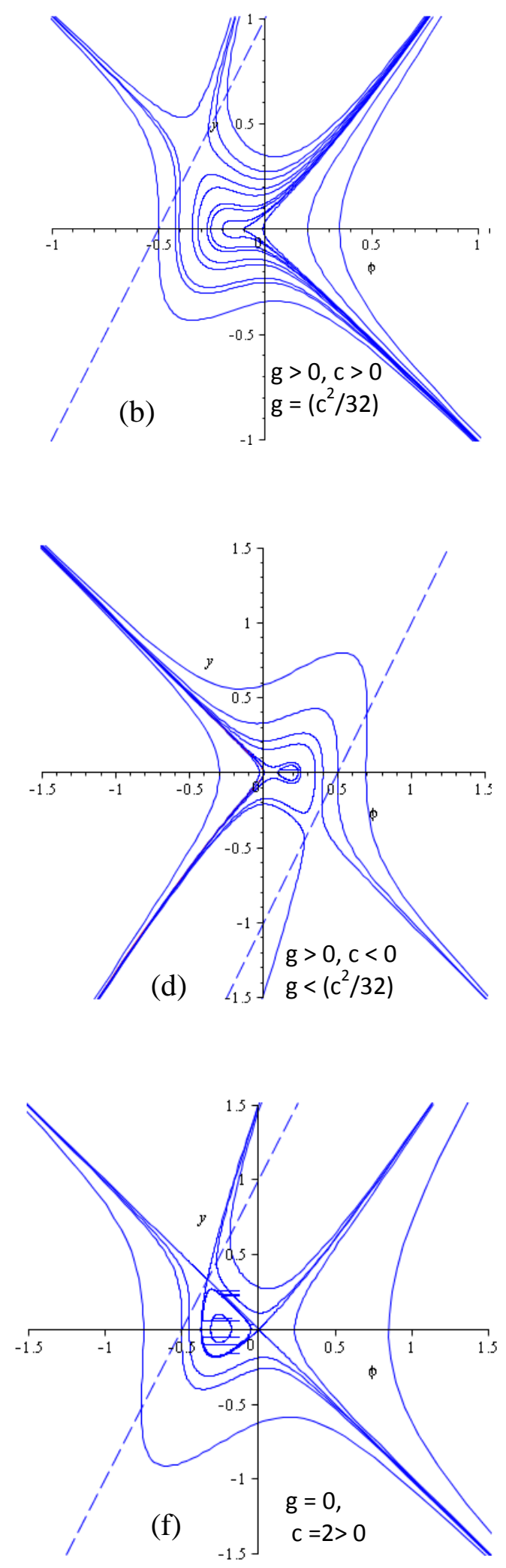


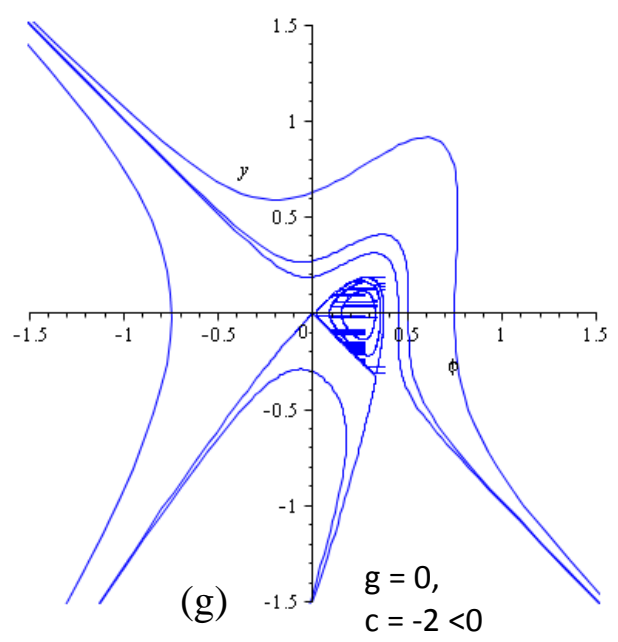

Figure 6: The phase portraits (y plotted against $\phi$ ) of Equation (3.8). Dotted line is the singular line $c+4 \phi-2 y=0$.

By using the above results we can draw the phase portraits of (3.8), depending on the parameter $(g, c)$. The phase portraits of (3.8) are shown in Figure 6.

From Figure 6, we see that there are no closed orbits adjacent to the singular straight line $c+4 \phi-2 y=0$ which, as they limit to the singular straight line $c+4 \phi-2 y=0$, could give us singular solutions. And from the discussion and theorems of the previous chapter, in the absence of either closed arched curves or curved triangles, once again neither singular peakons nor cuspons are possible.

\subsection{Phase portrait/possible solutions of Equation (1.6)}

Equation (1.6) can be written as,

$$
u_{t}-u_{x x t}=D_{x}\left(u^{2} u_{x x}-u_{x}^{2} u_{x x}+u u_{x}^{2}-u^{3}\right)
$$


Now let $u(x, t)=\phi(x-c t)=\phi(z)$, where $z=x-c t$ and $\mathrm{c}$ is the wave speed.

Then equation (3.10) becomes

$$
-c \frac{d \phi}{d z}+c \frac{d^{3} \phi}{d z^{3}}=\frac{d}{d z}\left[\phi^{2} \frac{d^{2} \phi}{d z^{2}}+\phi\left(\frac{d \phi}{d z}\right)^{2}-\left(\frac{d \phi}{d z}\right)^{2} \frac{d^{2} \phi}{d z^{2}}-\phi^{3}\right]
$$

Integrating (3.11) with respect to $\mathrm{z}$,

$$
-c \phi+c \frac{d^{2} \phi}{d z^{2}}=\phi^{2} \frac{d^{2} \phi}{d z^{2}}+\phi\left(\frac{d \phi}{d z}\right)^{2}-\left(\frac{d \phi}{d z}\right)^{2} \frac{d^{2} \phi}{d z^{2}}-\phi^{3}-g
$$

where $\mathrm{g}$ is the constant of integration.

Equation (3.12) is equivalent to the following 2-dimensional system

$$
\frac{d \phi}{d z}=y, \quad \frac{d y}{d z}=\frac{\phi y^{2}+c \phi-g-\phi^{3}}{c-\phi^{2}+y^{2}}
$$

Equation (3.13) belongs to the second type of singular traveling wave system described in section 2.2.3. Here $c-\phi^{2}+y^{2}=0$ defines the set of real planar curves such that the second part of the equation (3.13) is discontinuous on these curves. When the phase point $(\phi, y)$ passes through every branch of $c-\phi^{2}+y^{2}=0, \frac{d y}{d z}$ changes sign.

Let us make the transformation $d z=\left(c-\phi^{2}+y^{2}\right) d \zeta$, for $c-\phi^{2}+y^{2} \neq 0$, then equation (3.13) becomes

$$
\frac{d \phi}{d \zeta}=y\left(c-\phi^{2}+y^{2}\right), \quad \frac{d y}{d \zeta}=-g+\phi\left[y^{2}-\phi^{2}+c\right]
$$


Equation (3.14) is the associated regular system of Equation (3.13).

Let $P(\phi)=\phi^{3}-c \phi+g$ and $P^{\prime}(\phi)=3 \phi^{2}-c$. For a fixed $c>0$ the following hold:

1) $g>0$. When $g>\frac{2 c}{3} \sqrt{\frac{c}{3}}, P(\phi)$ only has a negative zero $z_{3}<-\sqrt{\frac{c}{3}}$; when $g=\frac{2 c}{3} \sqrt{\frac{c}{3}}$,

$P(\phi)$ has one simple zero $z_{3}$ and a double zero $z_{2,1}=\sqrt{\frac{c}{3}}$; when $g<\frac{2 c}{3} \sqrt{\frac{c}{3}}, P(\phi)$ has three simple zeros $z_{3}<0<z_{2}<\sqrt{\frac{c}{3}}<z_{1}<\sqrt{c}$.

2) $g<0$. When $|g|>\frac{2 c}{3} \sqrt{\frac{c}{3}}, P(\phi)$ has a positive zero $z_{1}>\sqrt{\frac{c}{3}}$; when $|g|=\frac{2 c}{3} \sqrt{\frac{c}{3}}, P(\phi)$ has only one simple zero $z_{1}$ and a double zero $z_{23}=-\sqrt{\frac{c}{3}}$; when $|g|<\frac{2 c}{3} \sqrt{\frac{c}{3}}, P(\phi)$ has three simple zeros $-\sqrt{c}<z_{3}<-\sqrt{\frac{c}{3}}<z_{2}<0<\sqrt{\frac{c}{3}}<z_{1}$.

Suppose $M\left(z_{i}, 0\right)$ be the coefficient matrix of the linearized system (3.14) at the critical point $S_{i}\left(z_{i}, 0\right)$. Then,

$$
J\left(z_{i}, 0\right)=\operatorname{det} M\left(z_{i}, 0\right)=\left(c-z_{i}^{2}\right)\left(3 z_{i}^{2}-c\right)
$$

According to planar dynamical systems theory, for a critical point of a planar integrable system, if $J<0$, then the critical point is a saddle, if $J>0$, then it is a center point. Let us define $h_{i}=H\left(z_{i}, 0\right), i=1,2,3$, where $H$ is the first integral of the system (3.14). It is clear from equation (3.14) that when $g>\frac{2 c}{3} \sqrt{\frac{c}{3}}\left(\right.$ or $\left.|g|>\frac{2 c}{3} \sqrt{\frac{c}{3}}, g<0\right)$, the unique critical point $S_{3}\left(z_{3}, 0\right)$ of (3.15) is a saddle point, where $g^{*}<g<\frac{2 c}{3} \sqrt{\frac{c}{3}}$ the point $S_{1}\left(z_{1}, 0\right)$ is a center and $S_{2}\left(z_{2}, 0\right)$ is 
a saddle point, where the value $g^{*}$ of $g$ is defined such that the homoclinic orbit of Equation (3.14) determined by the first integral $H(\phi, y)=h_{2}$ to the saddle point $S_{2}\left(z_{2}, 0\right)$ passes through the point $S_{s}(\sqrt{c}, 0)$, with $h_{2}=\frac{1}{4} g^{*} \sqrt{c}$.

3) If $g=0$, for both $c>0$ and $c<0$ the equilibrium point $(0,0)$ is a saddle point.

By using the above results we can draw the phase portrait of (3.14), depending on the parameters $(g, c)$. The phase portraits of (3.14) are shown in Figure 7 where the graph of the hyperbola $\phi^{2}-y^{2}=c$ is also shown Figure $7(\mathrm{~b})$. We note that, unlike the case of having singular straight line as in system (2.12), for the system (3.14) the hyperbola $\phi^{2}-y^{2}=c$ is not a solution of (3.14). For every fixed $c>0$, when $0<g<g^{*}$, system (3.14) has got a family of periodic orbits defined by the first integral $H(\phi, y)=h$. There exists homoclinic orbit of system (3.14) defined by $H(\phi, y)=h_{2}$, which transversely intersect the hyperbola $\phi^{2}-y^{2}=c$ at two points, $P_{ \pm}\left(\phi_{S^{\prime}} \pm y_{S}\right)$, where $\phi_{S}=\frac{h_{2}}{g}$ and $y_{s}=\sqrt{c-\left(\frac{h_{2}}{g}\right)^{2}}$.

Now, theorem 2 can be seen (following the details in [7]) to apply to the closed orbits adjacent to the singular hyperbola $\phi^{2}-y^{2}=c$ in Figure 7(b).

In particular, note that these closed orbits adjacent to the singular hyperbola have the arched curve form seen earlier in Figure 2(b). However, the existence of the periodic cusp waves or cuspons to which they gave rise (see theorem 3(2) in chapter 2) rested on theorem 1 in chapter 2 which holds only for the singular traveling wave equations of the first type (2.12). Since our singular traveling wave equation (3.13) is of the second type, viz (2.19) we generalize theorem 1, in chapter 2 to apply to our Equation (3.13). 
Generalized theorem 1 for (3.13): Theorem 1 applies to system (3.13)

Proof: Near the singular hyperbola $\phi^{2}-y^{2}=\mathrm{c}$ of (3.13) assume $\phi_{\mathrm{s}}-\phi=\varepsilon, \quad$ where $\phi_{\mathrm{s}}= \pm \sqrt{\mathrm{y}^{2}+\mathrm{c}}$. Then, Equation (3.13) may be written as

$$
\frac{\mathrm{d} \phi}{\mathrm{dz}}=\mathrm{y}, \quad \frac{\mathrm{dy}}{\mathrm{dz}}=\frac{-\mathrm{g}-\mathrm{c} \sqrt{\mathrm{y}^{2}+\mathrm{c}}+\mathrm{O}(\varepsilon)}{2 \varepsilon \sqrt{\mathrm{y}^{2}+\mathrm{c}}}
$$

Dividing, we have

$$
\frac{\mathrm{d} \phi}{\mathrm{dy}}\left(-\mathrm{g}-\mathrm{c} \sqrt{\mathrm{y}^{2}+\mathrm{c}}+\mathrm{O}(\varepsilon)\right)=2 \varepsilon \mathrm{y} \sqrt{\mathrm{y}^{2}+\mathrm{c}}
$$

Now, considering the closed periodic arched curve orbit $\gamma$ near the singular hyperbola $\phi^{2}-y^{2}=$ $\mathrm{c}$ in Figure 7(b), the first equation above gives the periodic as

$$
\mathrm{T}=\oint \frac{\mathrm{d} \phi}{\mathrm{y}}=\int_{\text {straight }} \frac{\mathrm{d} \phi}{\mathrm{y}}+\int_{\text {arched curve }} \frac{\mathrm{d} \phi}{\mathrm{y}}=\mathrm{T}_{1}+\mathrm{T}_{2}
$$

The contribution over the straight segment BA where the ordering $\phi_{\mathrm{s}}-\phi=\varepsilon$ holds is

$$
\mathrm{T}_{1}=\int_{\mathrm{Y}_{\mathrm{B}}}^{\mathrm{Y}_{\mathrm{A}}} \frac{\mathrm{d} \phi}{\mathrm{y}}=\int_{\mathrm{Y}_{\mathrm{B}}}^{\mathrm{Y}_{\mathrm{A}}} \frac{\mathrm{d} \phi}{\mathrm{y}} \frac{\mathrm{dy}}{\mathrm{dy}}=\int_{\mathrm{Y}_{\mathrm{B}}}^{\mathrm{Y}_{\mathrm{A}}} \frac{2 \varepsilon y}{\mathrm{y}\left[-\mathrm{g}-\mathrm{c} \sqrt{\mathrm{y}^{2}+\mathrm{c}}+\mathrm{O}(\varepsilon)\right]} d y
$$

using the expression for $\frac{d \phi}{d y}$. Now, in general $-g-c \sqrt{y^{2}+c} \neq 0$ along the segment BA adjacent to the hyperbola (except at the two end points). Hence, the denominator is of $O(1)$ and $\mathrm{T}_{1}=O(\varepsilon)$.

Thus, the rapid jump property along the singular hyperbola $\phi^{2}-y^{2}=c$ in Figure $7(b)$ holds. In conjunction with theorem 2 in chapter 2 , this implies the existence of periodic cusp waves or cuspons of Equation (3.13). 
(a)
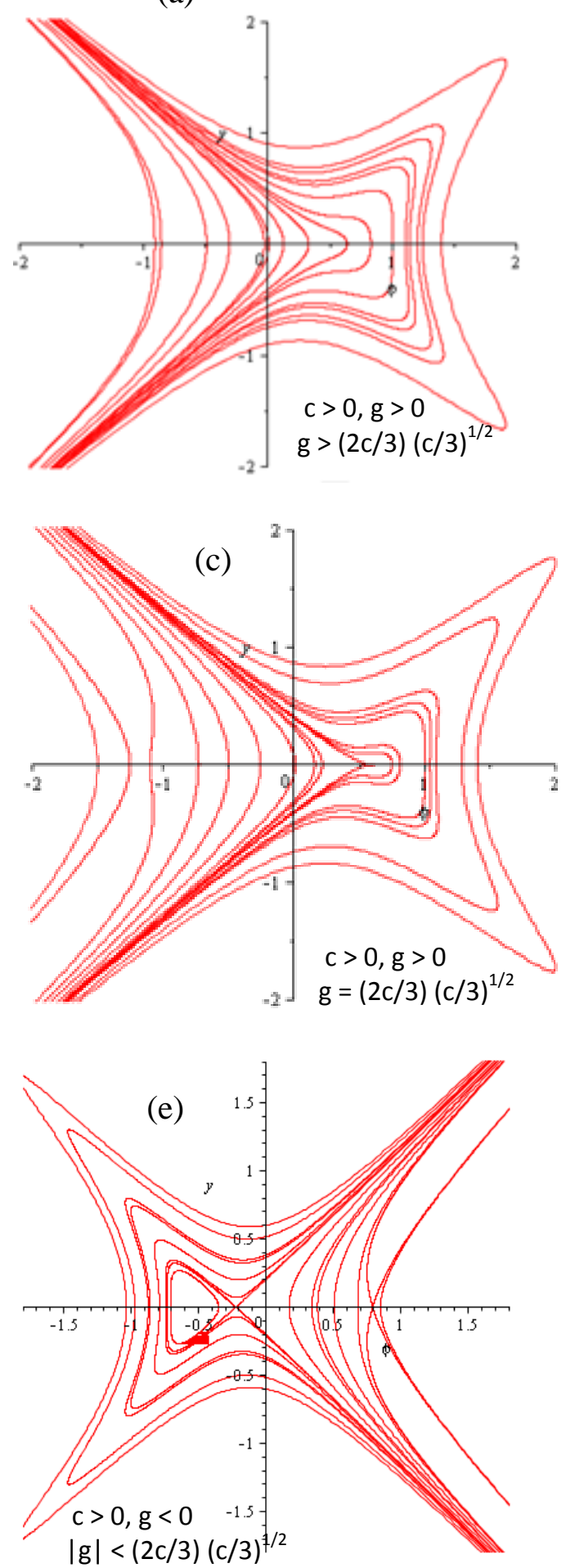
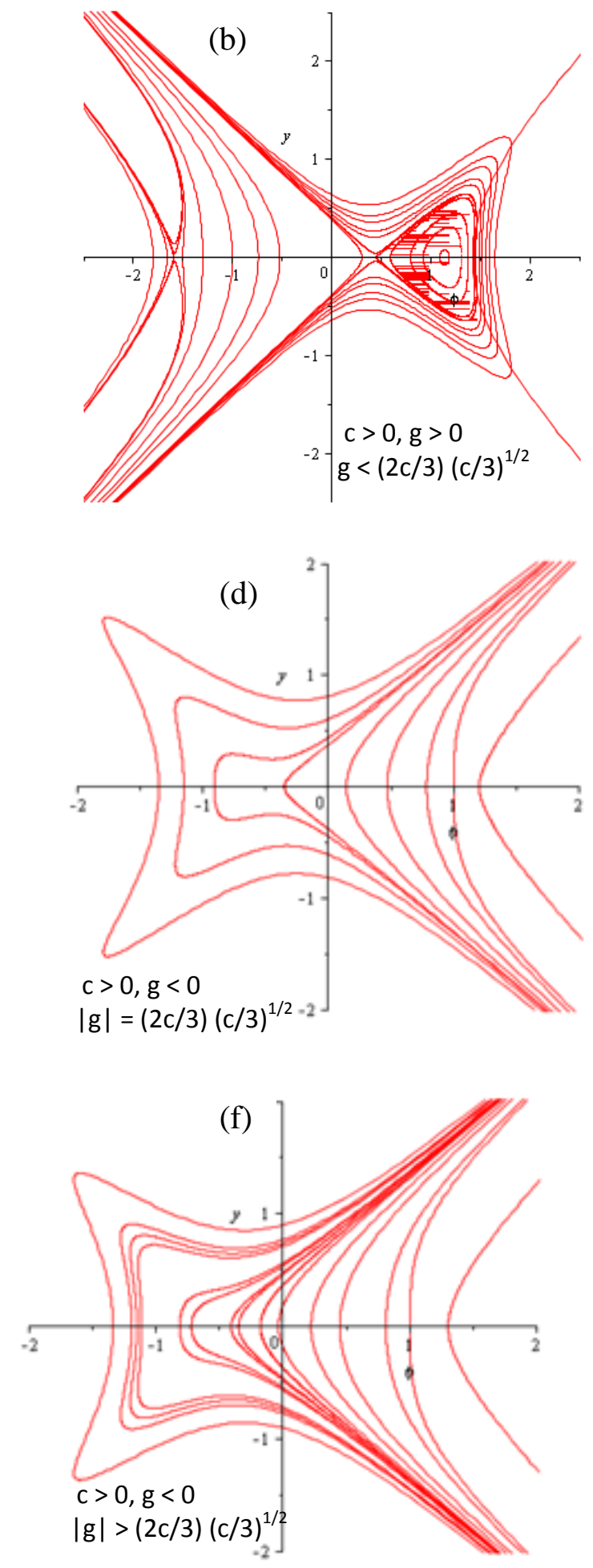

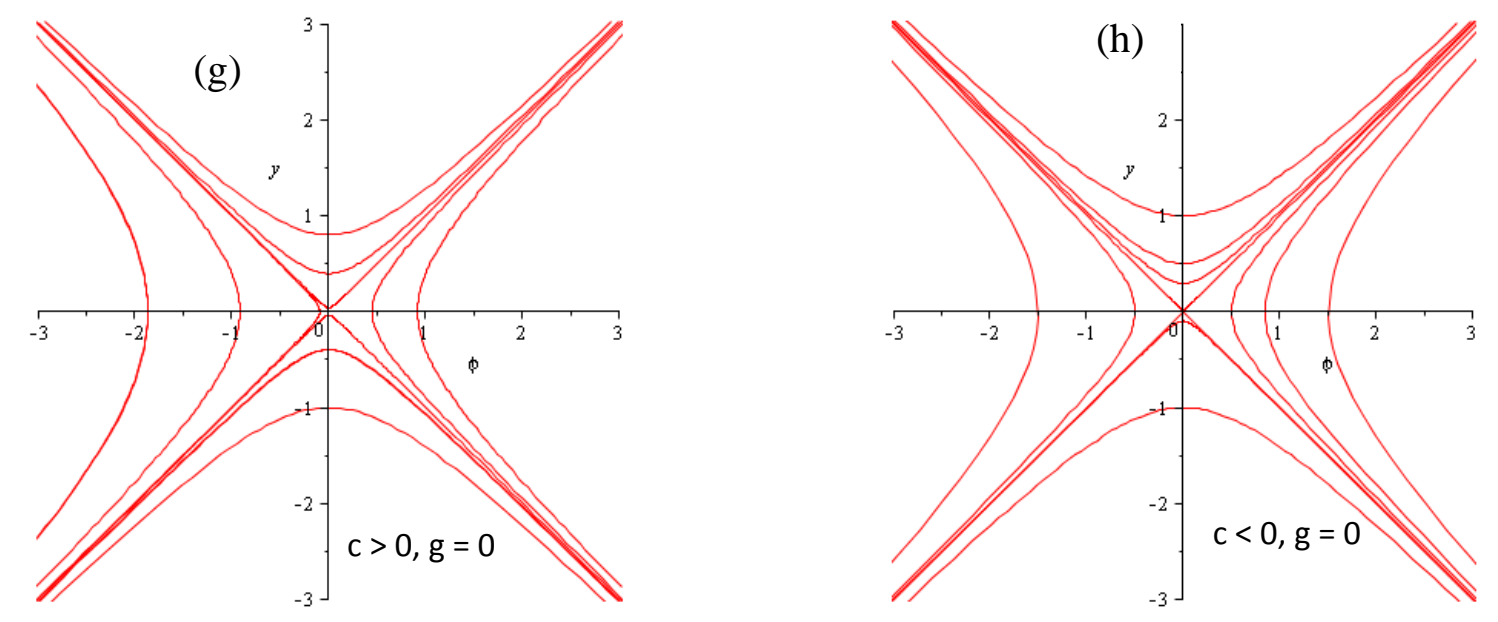

Figure 7: The phase portraits (y plotted against $\phi$ ) of Equation (3.14).

The profiles of the waves are as in Figure 3, and the jumps occur each time $\phi \rightarrow \phi_{\mathrm{s}}= \pm \sqrt{\mathrm{y}^{2}+\mathrm{c}}$ as the orbits cycle along the arched curves adjacent to $\phi=\phi_{\mathrm{s}}= \pm \sqrt{\mathrm{y}^{2}+\mathrm{c}}$ in Figure 7 (b).

Unlike for the system (3.14), for the system (3.13), the hyperbola $\phi^{2}-y^{2}=c$ is a singular curve of the vector field of the system. We consider $\phi>0$, when $\xi$ varied along the loop orbit defined by $H(\phi, y)=h_{2}$, the vector field of the system (3.13) has different direction on both the left hand side and right hand side of the hyperbola $\phi^{2}-y^{2}=c$. In fact on the right hand side of the hyperbola $\phi^{2}-y^{2}=c$ in the $1^{\text {st }}$ quadrant, one has $\frac{d \phi}{d z}=y>0, \frac{d y}{d z}=\frac{\phi y^{2}+c \phi-g-\phi^{3}}{c-\phi^{2}+y^{2}}>$ 0. This implies that the loop orbit of system (3.14) consists of three breaking solution of the system.

Thus we have the following result [10]

1) For any fixed $c>0$ when $0<g<g^{*}$, equation (1.6) has a M-shape wave solution defined by the branch of the level curves $H(\phi, y)=h_{2}$, which consists of three breaking wave solutions. 
2) For any fixed $c>0$ when $g^{*}<g<0$, equation (1.6) has W-shape wave solution defined by branch of level curves $H(\phi, y)=h_{2}$, which consists of three breaking wave solutions.

The infinite series solution for the homoclinic orbit, as seen in Figure 7(b) and 7(e) will be calculated in chapter 4 .

\subsection{Phase portrait/possible solutions of Equation (1.7)}

Equation (1.7) can be simplified and written as

$$
u_{t}-u_{x x t}=\frac{\partial}{\partial x}\left[4 u^{2}-2 u_{x}^{2}-2 u u_{x x}\right]
$$

Now, let $u(x, t)=\phi(x-c t)=\phi(z)$, where $z=x-c t$ and $c$ is the wave speed. Then we have the following

$$
u_{t}=-c \frac{d \phi}{d z}, \quad u_{x}=\frac{d \phi}{d z}, \quad u_{x x}=\frac{d^{2} \phi}{d z^{2}}, \quad u_{x x t}=-c \frac{d^{3} \phi}{d z^{3}}
$$

Then equation (3.16) becomes,

$$
-c \frac{d \phi}{d z}+c \frac{d^{3} \phi}{d z^{3}}=\frac{d}{d z}\left[4 \phi^{2}-2 \phi \frac{d^{2} \phi}{d z^{2}}-2\left(\frac{d \phi}{d z}\right)^{2}\right]
$$

Integrating this equation once with respect to $\mathrm{z}$,

$$
-c \phi+c \frac{d^{2} \phi}{d z^{2}}=\left[4 \phi^{2}-2 \phi \frac{d^{2} \phi}{d z^{2}}-2\left(\frac{d \phi}{d z}\right)^{2}+g\right]
$$

where, $\mathrm{g}$ is the constant of integration.

Equation (3.17) is equivalent to the following 2-dimensional system 


$$
\frac{d \phi}{d z}=y, \quad \frac{d y}{d z}=\frac{4 \phi^{2}-2 y^{2}+c \phi+g}{c+2 \phi}
$$

which is the traveling wave system for Equation (1.5)

Equation (3.18) belongs to the first type of singular traveling wave system (2.12) as described in chapter 2 . In the straight line $\phi=-\frac{c}{2}$, of the $(\phi, y)$ phase plane, the second equation of (3.18) is discontinuous. The straight line $\phi=-\frac{c}{2}$ is the singular straight line described in the previous chapter.

As described in chapter 2 , to avoid this discontinuity on the singular straight line we make the transformation $d z=(c+2 \phi) d \zeta$. Under this transformation Equation (3.18) becomes,

$$
\frac{d \phi}{d \zeta}=y(c+2 \phi), \quad \frac{d y}{d \zeta}=4 \phi^{2}-2 y^{2}+c \phi+g
$$

System of Equation (3.19) is the associated regular system of Equation (3.18). Since the first integral of both Equations (3.18) and (3.19) are same, therefore both of them have the same phase orbit, except on the straight line $\phi=-\frac{c}{2}$.

The system of Equation (3.19) has the following equilibrium points: $z_{1}=\left(\frac{-c-\sqrt{c^{2}-16 g}}{8}, 0\right), z_{2}=\left(\frac{-c+\sqrt{c^{2}-16 g}}{8}, 0\right), z_{3}=\left(\frac{-c}{2},-\frac{\sqrt{c^{2}+2 g}}{2}\right)$ and $z_{4}=\left(\frac{-c}{2}, \frac{\sqrt{c^{2}+2 g}}{2}\right)$. Here, $z_{1}$ and $z_{2}$ are called the regular equilibrium points, while $z_{3}$ and $z_{4}$ are called the singular equilibrium points.

We study the stability of these equilibrium points using the linearized system of Equation (3.19). 
When $c>0$ and $0<g<\frac{c^{2}}{16}$, the equilibrium point $z_{1}$ is a center, while the equilibrium points $z_{2}, z_{3}$ and $z_{4}$ are saddle points. The phase portrait is drawn in Figure 8(a).

When $c>0, g<0$ and $|g|<\frac{c^{2}}{2}$, the equilibrium point $z_{1}$ is a center, while the equilibrium points $z_{2}, z_{3}$ and $z_{4}$ are saddle points. The phase portrait is drawn in Figure 8(b)

When $c<0$ and $0<g<\frac{c^{2}}{16}$, the equilibrium points $z_{1}, z_{3}$ and $z_{4}$ are saddle points, while $z_{2}$ is a center. The phase portrait is drawn in Figure 8 (c).

When $\mathrm{c}<0, g<0$ and $|g|<\frac{c^{2}}{2}$, the equilibrium points $z_{1}, z_{3}$ and $z_{4}$ are saddle points, while $z_{2}$ is a center. The phase portrait is drawn in Figure 8 (d)

If we consider the constant of integration ' $g$ ' of Equation (3.17) to be zero then, the system of Equation (3.19) has equilibrium points, $z_{1}=\left(-\frac{c}{2},-\frac{c}{2}\right), z_{2}=\left(-\frac{c}{2}, \frac{c}{2}\right), z_{3}=(0,0)$ and $z_{4}=\left(-\frac{c}{4}, 0\right)$. Here $z_{1}, z_{2}$ are the singular equilibrium points, while $z_{3}, z_{4}$ are the regular equilibrium points.

The eigenvalue of the Jacobian of the Equation (3.19) with $\mathrm{g}=0$ at the fixed point $z_{1}=\left(-\frac{c}{2},-\frac{c}{2}\right)$ are $2 \mathrm{c}$ and $-\mathrm{c}$, thus for both $\mathrm{c}>0$ and $\mathrm{c}<0$, the fixed point $z_{1}=\left(-\frac{c}{2},-\frac{c}{2}\right)$ is a saddle point.

The eigenvalue of the Jacobian of Equation (3.19) with $\mathrm{g}=0$ at the fixed point $z_{2}=$ $\left(-\frac{c}{2}, \frac{c}{2}\right)$ are $-2 \mathrm{c}$ and $\mathrm{c}$, thus for both $\mathrm{c}>0$ and for $\mathrm{c}<0$, the fixed point $z_{2}=\left(-\frac{c}{2}, \frac{c}{2}\right)$ is a saddle point. 
The eigenvalue of the Jacobian of Equation (3.19) with $\mathrm{g}=0$ at the fixed point $z_{3}=$ $(0,0)$ are $\mathrm{c}$ and $-\mathrm{c}$, thus for both $\mathrm{c}>0$ and for $\mathrm{c}<0$, the fixed point $z_{3}=(0,0)$ is a saddle point.

The real part of the eigenvalue of the Jacobian of Equation (3.19) with $g=0$ at the fixed point $z_{4}=\left(-\frac{c}{4}, 0\right)$ is zero, thus for $\mathrm{c}>0$ and for $\mathrm{c}<0$, the fixed point $z_{4}=\left(-\frac{c}{4}, 0\right)$ is a center.

The phase portrait of Equation (3.19) for $\mathrm{g}=0$ are drawn in Figure 8(e) and Figure 8(f) for $\mathrm{c}>0$ and $\mathrm{c}<0$ respectively.

Now, theorem 2 can be seen (following the details in [7]) to apply to the closed orbits adjacent to the singular straight line $\phi=\phi_{\mathrm{s}}=-\frac{\mathrm{c}}{2}$ in Figure 8(b), 8(d), 8(e) and Figure 8(f).

In particular in Figure 8(e) and 8(f), note that these closed orbits adjacent to the singular straight line, have the curve triangular form seen earlier in Figure 2(a). We notice that, the singular traveling wave Equation (3.18) is of the first type (2.12) as discussed in chapter 2. Thus from theorem 3 in chapter 2 we can conclude that these curve triangles give rise to solitary cusp waves of the peak type called peakons.

Similarly in Figure 8(b) and 8(d), we notice that these closed orbits adjacent to the singular straight line, have the arched curve form seen earlier in Figure 2(b). Thus from theorem 3 in chapter 2 we conclude that, these arch curve give rise to a periodic cusp waves of the peak type called cuspons. 

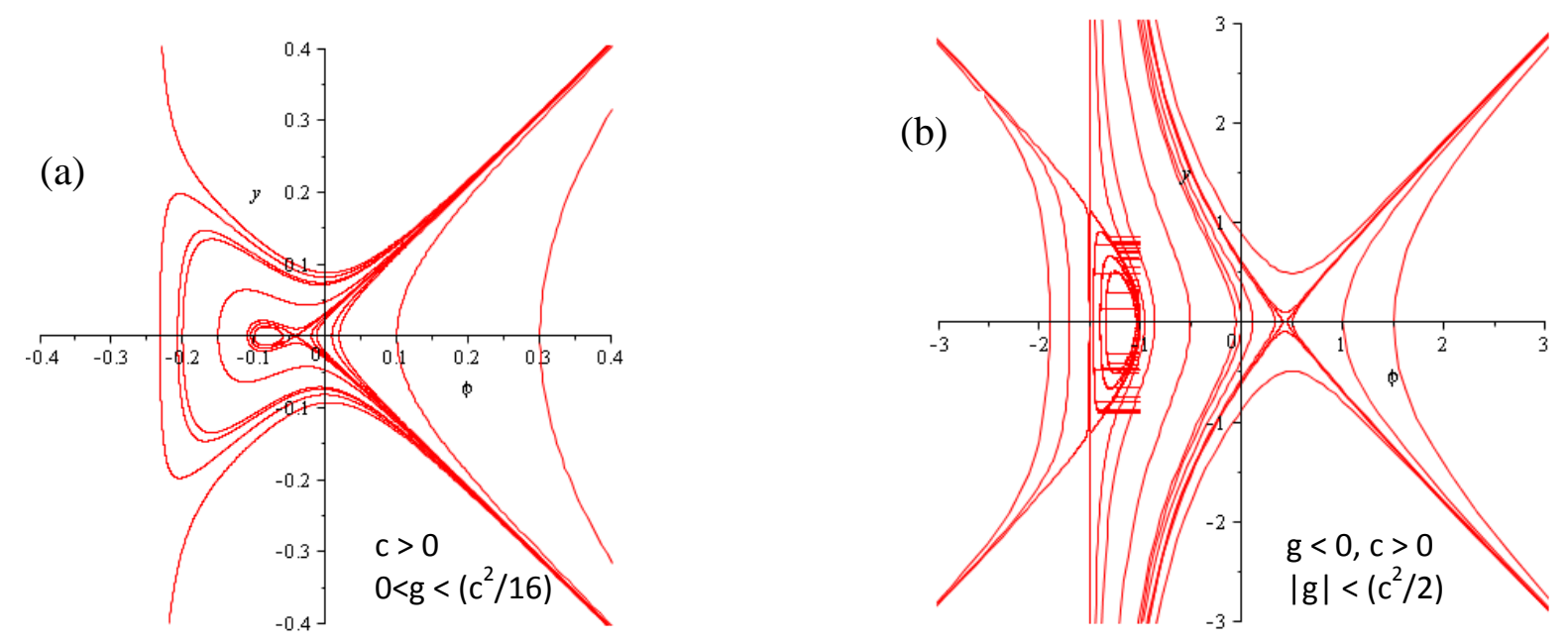

(c)
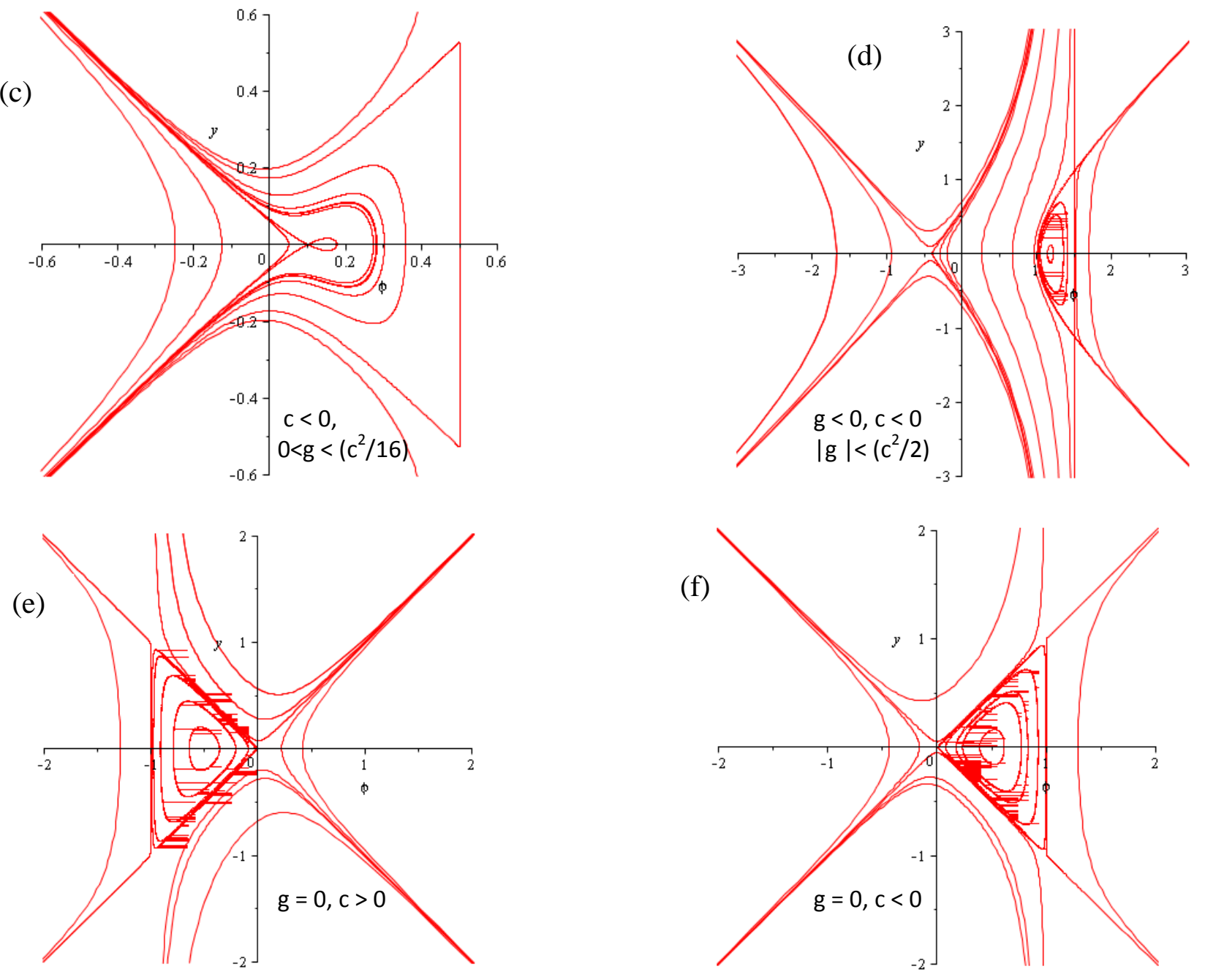

Figure 8: The phase portraits (y plotted against $\phi$ ) of Equation (3.19). 


\section{CHAPTER 4: ANALYTIC SOLUTIONS FOR HOMOCLINIC AND HETEROCLINIC ORBITS OF GENERALIZED CAMASSA HOLM EQUATIONS}

In this chapter, we change gears and consider convergent, multi-infinite, series solutions for the possible homoclinic and heteroclinic orbits of the generalized Camassa-Holm Equations $(1.4)-(1.7)$.

Homoclinic orbits of dynamical systems have been widely treated in recent years by a variety of approaches. For instance, an early review integrating bifurcation theoretical and numerical approaches was given in [11]. Homoclinic orbits are important in applications for a variety of reasons. In the context of ODE systems, they are often anchors for the local dynamics in their vicinity. Under certain conditions, their existence may indicate the existence of chaos in their neighborhood $[12,13]$. In a totally different setting, if the governing dynamical system is the traveling-wave ODE for a partial differential equation or equations, its homoclinic orbits correspond to the solitary wave or pulse solutions of the PDEs, which have many important uses and applications in nonlinear wave propagation theory, nonlinear optics, and in various other settings [11].

We employ a recently developed approach $[8,9]$, using the method of undetermined coefficients to derive heteroclinic and homoclinic orbits of Equations (3.2), (3.6), (3.12) and (3.17). Convergent analytic series for these orbits corresponding to pulse/front solutions of the generalized CH Equations (1.4)-(1.7) are derived and investigated here. 


\subsection{Infinite Series for heteroclinic orbits of Equation (3.2)}

Equation (3.2) can be written as

$$
-c \phi+c \phi^{\prime \prime}=2 \phi^{2}-2 \phi \phi^{\prime \prime}-2 \phi^{\prime 2}+2 \phi^{\prime} \phi+g
$$

where the ' indicates the derivative with respect to $z$.

The Equation (4.1) is non-reversible under the standard reversibility of classical mechanical systems:

$$
z \rightarrow-z,\left(\phi, \phi^{\prime}, \phi^{\prime \prime}\right) \rightarrow\left(\phi,-\phi^{\prime}, \phi^{\prime \prime}\right)
$$

Mathematically, this would translate to solutions having odd parity in $z$, but solutions of Equation (4.1) would not display this property.

Let us consider a parameter range in which Equation (3.4) admits a heteroclinic orbit.

When $\mathrm{c}>0$ and $0<g<\frac{c^{2}}{8}$ the equilibrium $z_{1}=\left(\frac{-c+\sqrt{c^{2}-8 g}}{4}, 0\right)$ is a saddle and the equilibrium $z_{2}=\left(\frac{-c-\sqrt{c^{2}-8 g}}{4}, 0\right)$ is a stable node. A heteroclinic orbit joins $z_{1}$ and $z_{2}$ in this parameter range, for example when $\mathrm{c}=3$ and $\mathrm{g}=1.1$ as shown in Figure 5(a). Let us now proceed to construct the series solution for the heteroclinic orbit joining two equilibrium points $x_{1}$ and $x_{2}$.

We look for a solution of the following form:

$$
\phi(z)= \begin{cases}\phi^{+}(z)=x_{2}+\sum_{k=1}^{\infty} a_{k} e^{k \alpha z} & z>0 \\ 0 & z=0 \\ \phi^{-}(z)=x_{1}+\sum_{k=1}^{\infty} b_{k} e^{k \beta z} & z<0\end{cases}
$$


where $x_{1}=\frac{-c-\sqrt{c^{2}-8 g}}{4}, x_{2}=\frac{-c+\sqrt{c^{2}-8 g}}{4}$. Moreover $\alpha<0$ and $\beta>0$ are undetermined constant and $a_{k}, b_{k}, k \geq 1$ are, at the outset, arbitrary coefficients. From the expression for $\phi^{+}(z)$ it follows that the terms into the equation (4.1) can be written as:

$$
\begin{gathered}
\phi^{+}(z)=x_{2}+\sum_{k=1}^{\infty} a_{k} e^{k \alpha z} \\
\phi^{\prime}=\sum_{k=1}^{\infty} a_{k} k \alpha e^{k \alpha z} \\
\phi^{\prime \prime}=\sum_{k=1}^{\infty} a_{k} k^{2} \alpha^{2} e^{k \alpha z} \\
2 \phi^{2}=2 x_{2}^{2}+4 x_{2} \sum_{k=1}^{\infty} a_{k} e^{k \alpha z}+2 \sum_{k=2}^{\infty} \sum_{i=1}^{k-1} a_{k-i} a_{i} e^{k \alpha z} \\
-2 \phi^{2}+2 \phi^{\prime} \phi=2 x_{2} \sum_{k=1}^{\infty} k \alpha a_{k} e^{k \alpha z}+2 \sum_{k=2}^{\infty} \sum_{i=1}^{k-1}(k-i)(1-i \alpha) \alpha a_{k-i} a_{i} e^{k \alpha z} \\
-2 \phi \phi^{\prime \prime}=-2 x_{2} \sum_{k=1}^{\infty} k^{2} \alpha^{2} a_{k} e^{k \alpha z}-2 \sum_{k=2}^{\infty} \sum_{i=1}^{k-1}(k-i)^{2} \alpha^{2} a_{k-i} a_{i} e^{k \alpha z}
\end{gathered}
$$

Using (4.4)-(4.9) in the irreversible Equation (4.1) we have 


$$
\begin{array}{r}
\sum_{k=1}^{\infty}\left((k \alpha)^{2}\left(c+2 x_{2}\right)-2 x_{2} k \alpha-\left(c+4 x_{2}\right)\right) a_{k} e^{k \alpha z}-c x_{2}-2 x_{2}{ }^{2}-g \\
=2 \sum_{k=2}^{\infty} \sum_{i=1}^{k-1}\left[1+(k-i)(1-i \alpha) \alpha-(k-i)^{2} \alpha^{2}\right] a_{k-i} a_{i} e^{k \alpha z}
\end{array}
$$

As $-c x_{2}-2 x_{2}{ }^{2}-g=0$, comparing the coefficients of $e^{k \alpha z}$ for each $\mathrm{k}$ we obtain for $\mathrm{k}=1$ :

$$
\left(\alpha^{2}\left(c+2 x_{2}\right)-2 x_{2} \alpha-\left(c+4 x_{2}\right)\right) a_{1}=0
$$

Assuming $a_{1} \neq 0$ (otherwise $a_{k}=0$ for all $k>1$ by induction), results in the values:

$$
\alpha_{1,2}=\frac{x_{2} \pm \sqrt{x_{2}^{2}+\left(c+4 x_{2}\right)\left(c+2 x_{2}\right)}}{\left(c+2 x_{2}\right)}
$$

When $\mathrm{c}>0$ and $0<g<\frac{c^{2}}{8}$, the quantities $\alpha_{1,2}$ are both real and negative.

As our solution for (4.4) needs to converge for $z>0$, we indifferently can choose $\alpha_{1}$ or $\alpha_{2}$. Here we pick the negative root $\alpha_{1}=\frac{x_{2}-\sqrt{x_{2}^{2}+\left(c+4 x_{2}\right)\left(c+2 x_{2}\right)}}{\left(c+2 x_{2}\right)}$. Therefore,

$$
\phi^{+}(z)=x_{2}+\sum_{k=1}^{\infty} a_{k} e^{k \alpha_{1} z}
$$

For $k>1$, let for $k=2$ from Equation (4.10) we have

$$
F\left(k \alpha_{1}\right) a_{2}=2\left(1-2 \alpha_{1}{ }^{2}+\alpha_{1}\right) a_{1}^{2}
$$

where $F(k \alpha)=(k \alpha)^{2}\left(c+2 x_{2}\right)-2 x_{2} k \alpha-\left(c+4 x_{2}\right)$, thus 


$$
a_{2}=\frac{2\left(1-2 \alpha_{1}{ }^{2}+\alpha_{1}\right) a_{1}^{2}}{F\left(2 \alpha_{1}\right)}
$$

Similarly for $\mathrm{k}=3$,

$$
a_{3}=2 \sum_{i=1}^{2} \frac{\left[1+(3-i)\left(1-i \alpha_{1}\right) \alpha_{1}-(3-i)^{2} \alpha_{1}^{2}\right] a_{3-i} a_{i}}{F\left(3 \alpha_{1}\right)}
$$

For $\mathrm{k}>0$,

$$
a_{k}=2 \sum_{i=1}^{k-1} \frac{\left[1+(k-i)\left(1-i \alpha_{1}\right) \alpha_{1}-(k-i)^{2} \alpha_{1}^{2}\right] a_{k-i} a_{i}}{F\left(k \alpha_{1}\right)}
$$

Therefore the coefficients $a_{k}, k>1$ can be directly obtained from the Equation (4.16) as follows

$$
a_{k}=\varphi_{k} a_{1}^{k}
$$

where $\varphi_{k}, k>1$ are functions which can be obtained using equation (4.14)-(4.16) and depending on $\alpha_{1}$ and coefficients of the Equation (4.1).

The first part of the heteroclinic orbit corresponding to $z>0$ has thus been determined in terms of $a_{1}$ :

$$
\phi^{+}(z)=x_{2}+a_{1} e^{\alpha_{1} z}+\sum_{k=2}^{\infty} \varphi_{k} a_{1}^{k} e^{k \alpha_{1} z},
$$

We shall now construct the second part corresponding to $z<0$. Since the equation (4.1) is not reversible we do not have any symmetric property for the solution, therefore impose the following solution form: 


$$
\phi^{-}(z)=x_{1}+\sum_{k=1}^{\infty} b_{k} e^{k \beta z}
$$

where real part of $\beta>0$, because the solution $\phi^{-}(z)$ needs to converge for $z<0$. Working as for $z>0$, we obtain for $k=1$ the following equation

$$
\left(\left(\beta^{2}\left(c+2 x_{1}\right)-2 x_{1} \beta-\left(c+4 x_{1}\right)\right)\right) b_{1}=0
$$

Assuming $b_{1} \neq 0$ (otherwise $b_{k}=0$ for all $k>1$ by induction), we find the roots in Equation (4.20):

$$
\beta_{1,2}=\frac{x_{1} \pm \sqrt{x_{1}^{2}+\left(c+4 x_{1}\right)\left(c+2 x_{1}\right)}}{\left(c+2 x_{1}\right)}
$$

which are real and opposite in our parameter regime. We choose $\beta=\beta_{2}=\frac{x_{1}+\sqrt{x_{1}^{2}+\left(c+4 x_{1}\right)\left(c+2 x_{1}\right)}}{\left(c+2 x_{1}\right)}$ ,with $\beta_{2}>0$, since our solution (4.19) needs to converge for $\mathrm{z}<0$.

Similarly for $\mathrm{z}<0$, for $k>1$, we obtain the following equation

$$
F\left(k \beta_{2}\right) b_{k}=2 \sum_{i=1}^{k-1}\left[1+(k-i)\left(1-i \beta_{2}\right) \beta_{2}-(k-i)^{2} \beta_{2}^{2}\right] b_{k-i} b_{i}
$$

where the polynomial

$$
F\left(k \beta_{2}\right)=\left((k \beta)^{2}\left(c+2 x_{1}\right)-2 x_{1} k \beta-\left(c+4 x_{1}\right)\right)
$$

Analogously to the treatment for $z>0$ we obtain: 


$$
b_{k}=2 \sum_{i=1}^{k-1} \frac{\left[1+(k-i)\left(1-i \beta_{2}\right) \beta_{2}-(k-i)^{2} \beta_{2}^{2}\right] b_{k-i} b_{i}}{F\left(k \beta_{2}\right)}
$$

Thus, the series coefficients can be obtained as for $z<0$ as follows:

$$
b_{k}=\psi_{k} b_{1}^{k}
$$

where $\psi_{k}$ are given in terms of $b_{1}$ and the coefficient of equation (4.1).

We want to construct a solution continuous at $z=0$, therefore we impose

$$
\begin{aligned}
& \phi^{+}(0)=x_{2}+a_{1}+\sum_{k=2}^{\infty} \varphi_{k} a_{1}^{k}=0 \\
& \phi^{-}(0)=x_{1}+b_{1}+\sum_{k=2}^{\infty} \psi_{k} b_{1}^{k}=0
\end{aligned}
$$

and we choose $a_{1}$ and $b_{1}$ as the nontrivial solutions of the above polynomial equations (4.25) and (4.26).

Let us choose the parameter values as $c=-2$ and $g=0.2<\frac{c^{2}}{8}$ (See Figure 5(c)). In this case the equilibrium $x_{1}=0.1127$ is a saddle with eigenvalues $\beta_{1}=-1$ and $\beta_{2}=.8730$, and the equilibrium $x_{2}=0.8873$ is an unstable node with eigenvalues $\alpha_{1}=-1$ and $\alpha_{2}=-6.8730$. 

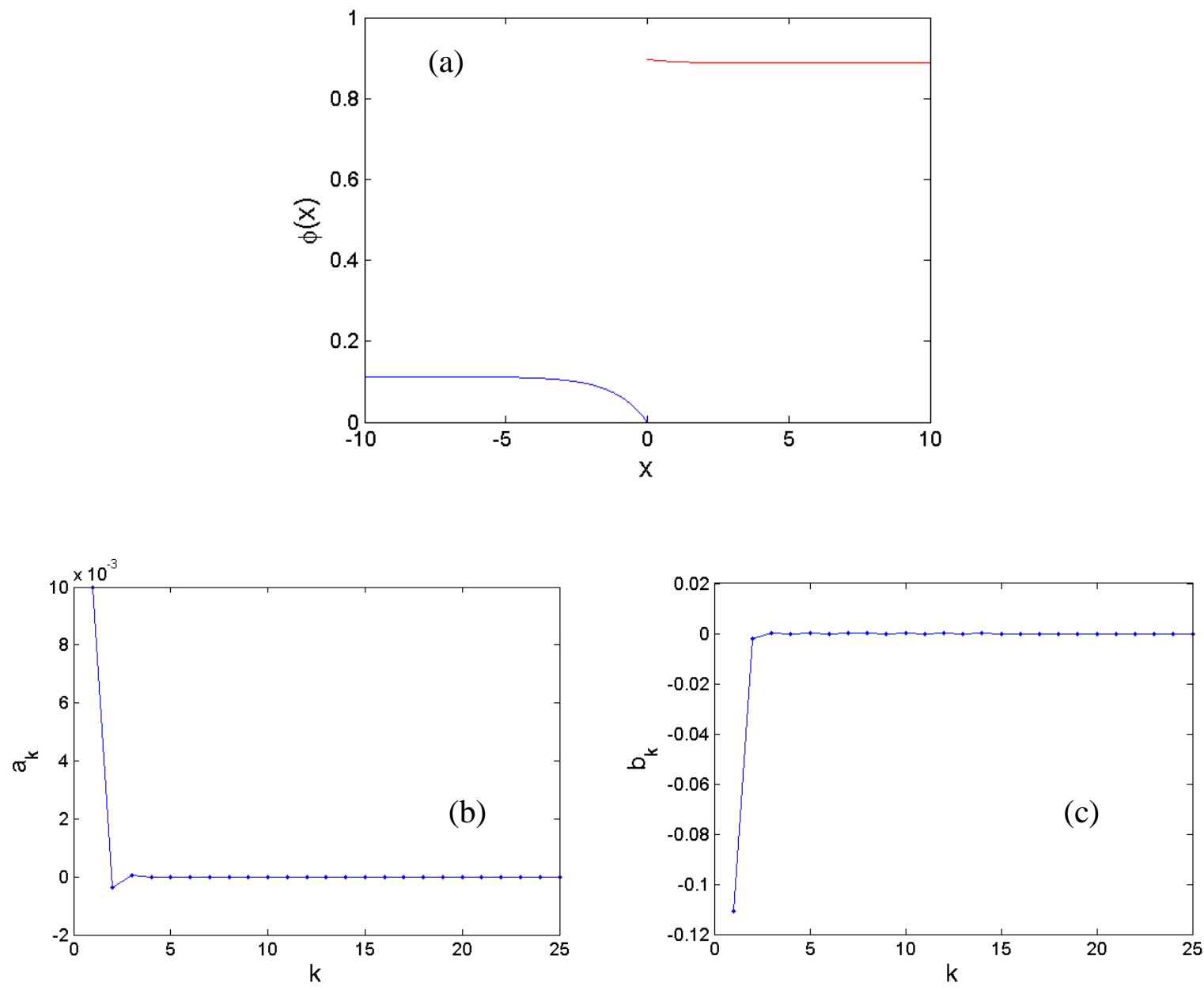

Figure 9: The parameters are chosen as $\mathrm{c}=-2$ and $\mathrm{g}=0.2$. (a) Discontinuous series solution for the heteroclinic orbit joining $x_{1}=0.1127$ and $x_{2}=0.8873$. (b) The series coefficients $a_{k}$ converge (c) The series coefficient $b_{k}$ converge.

The equation for the continuity (4.25) admits no solution on the right side, therefore we choose the arbitrary value $a_{1}=0.01$ which lead to the convergence of the series coefficients $a_{k}$ [see Figure 9 (b)], but the solution (4.3) is discontinuous from the right [see Figure 9(a)]. 

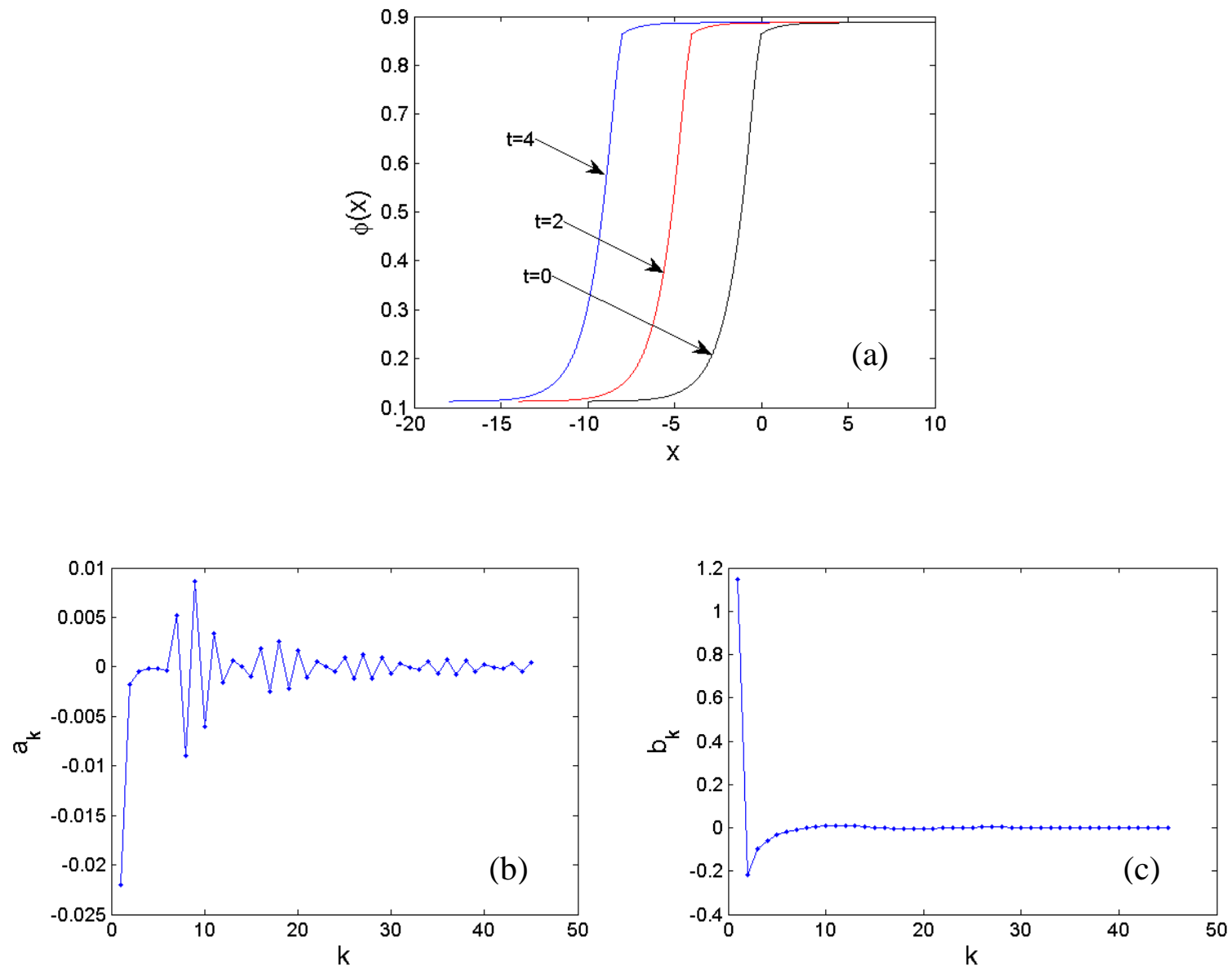

Figure 10: The parameters are chosen as $c=-2$ and $g=0.2$. (a) Series solution for the heteroclinic orbit joining $x_{1}=0.1127$ and $x_{2}=0.8873$. Here $\phi^{+}(0)=\phi^{-}(0)=0.8637$, (b) The series coefficients $a_{k}$ converge (c) The series coefficient $b_{k}$ converge.

The equation for the continuity (4.26) admits the solution $b_{1}=-0.1107$, which also lead to the convergence of the series coefficients $b_{k}$, see Figure 9 (c).

To obtain also the continuity, we choose $a_{1}=-0.022$ and $b_{1}=1.1479$, in such a way to preserving the convergence of the series coefficients $b_{k}$ and obtaining the continuity at the origin, imposing that $\phi^{-}(0)=\phi^{+}(0)=0.8637$. Figure 10(a) shows the continuous solution $\phi(x)$ 
versus $\mathrm{x}$ at different fixed instants t. In Figure 10 (c) the converging series of the coefficients $b_{k}$ is given. The series coefficients $a_{k}$ is as in Figure 10(b).

In Figure 10(a), the traveling wave nature of the solution of Equation (3.2) is shown for instance $\mathrm{t}=0, \mathrm{t}=2$, and $\mathrm{t}=4$.

Let us now choose the parameter values as $\mathrm{c}=3$ and $\mathrm{g}=1.1<\frac{c^{2}}{8}$ (See Figure $\left.5(\mathrm{a})\right)$. In this case the equilibrium $x_{1}=-.6382$ is a saddle with eigenvalues $\beta_{1}=-1$ and $\beta_{2}=0.2595$, and the equilibrium $x_{2}=-0.8612$ is an attractive stable node with eigenvalues $\alpha_{1}=-1$ and $\alpha_{2}=0.3504$.

We truncate the series solution at $M=25$. The equation for the continuity (4.25) admits no solution, therefore we choose the arbitrary value $a_{1}=0.18$ which lead to the convergence of the series coefficients $a_{k}$ (see Figure 11 (b)), but the solution (4.3) is discontinuous from the right [see Figure 11(a)]. The equation for the continuity (4.26) admits the solution $b_{1}=0.2350$, which also lead to the convergence of the series coefficients $b_{k}$, see Figure 11 (c).

To obtain also the continuity, we choose $b_{1}=0.0972$, in such a way to preserving the convergence of the series coefficients $b_{k}$ and obtaining the continuity at the origin, imposing that $\phi^{-}(0)=\phi^{+}(0)=-0.4991$. Figure 12(a) shows the continuous solution $\phi(\mathrm{x})$ versus $\mathrm{x}$ at different fixed instants t. In Figure 12 (b) the converging series of the coefficients $b_{k}$ is given. The series coefficients $a_{k}$ is as in Figure 11(b). 

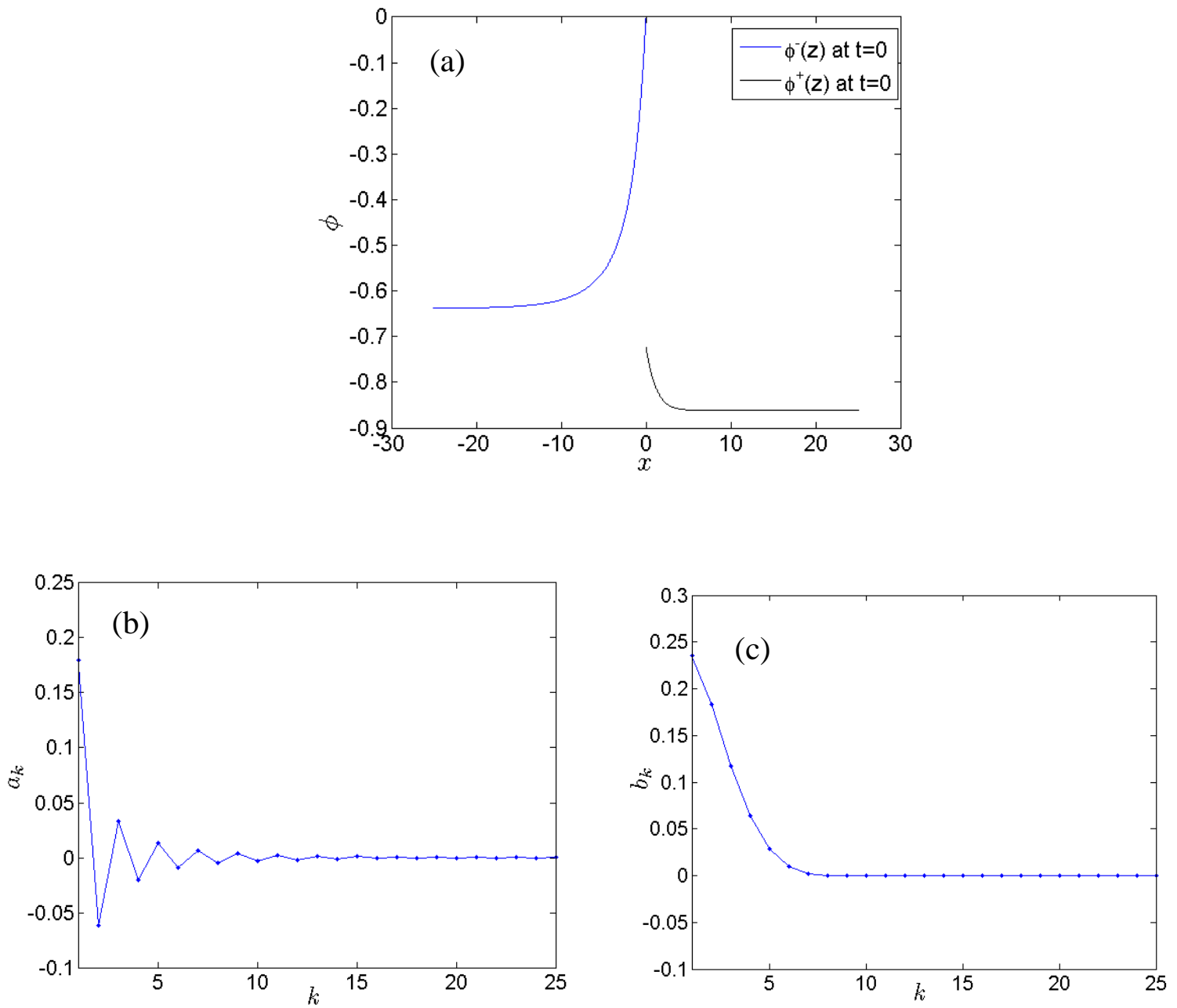

Figure 11: The parameters are chosen as $\mathrm{c}=3$ and $\mathrm{g}=1.1$. (a) Discontinuous series solution for the heteroclinic orbit joining $x_{1}=-0.6382$ and $x_{2}=-0.8612$. (b) The series coefficients $a_{k}$ converge (c) The series coefficient $b_{k}$ converge. 

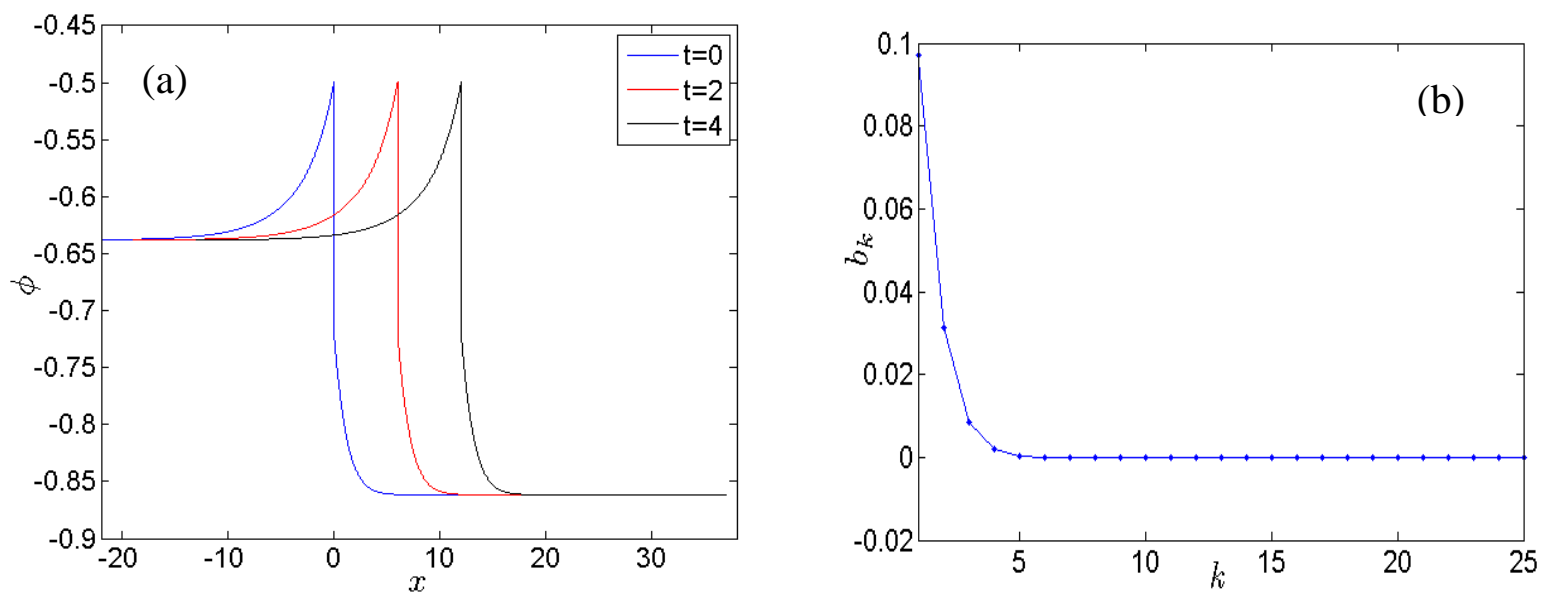

Figure 12: The parameters are chosen as $c=3$ and $g=1.1$. (a) Series solution for the heteroclinic orbit joining $x_{1}=-0.6382$ and $x_{2}=-0.8612$. Here $\phi^{+}(0)=\phi^{-}(0)=-0.4991$ (b) Converging series coefficient $b_{k}$ versus $\mathrm{k}$.

As can be seen in Figures 11-12(a), the solution is not symmetric where the positive portion of $\phi(\mathrm{x})$ decays faster $\left(\alpha_{1}=-1.0\right)$ comparing to that in the negative portion of $\mathrm{x}\left(\beta_{2}=\right.$ 0.2595). In Figure 12(a), the traveling wave nature of the solution of Equation (3.2) is shown for instance $\mathrm{t}=0, \mathrm{t}=2$, and $\mathrm{t}=4$.

\subsection{Infinite Series for homoclinic orbits of Equation (3.6)}

Equation (3.6) can be written as

$$
-c \phi+c \phi^{\prime \prime}=8 \phi^{2}-4 \phi \phi^{\prime \prime}-2 \phi^{\prime 2}+2 \phi^{\prime} \phi^{\prime \prime}+g
$$

where the ' indicates the derivative with respect to $\mathrm{z}$. 
As shown in section 3.2 the Equation (3.8) admits saddle points and homoclinic orbit to such points have been observed (see for example Figure 6(a), 6(c), 6(e)).

Let us now proceed to construct, at first formally, the series solution for the homoclinic orbit to such a point, here indicated with $x_{0}$. Suppose that for $z>0$ :

$$
\phi^{+}(z)=\sum_{k=1}^{\infty} a_{k} e^{k \alpha z}+x_{0}
$$

where $\alpha<0$ is an undetermined constant and, $a_{k}, k \geq 1$ are, at the outset, arbitrary coefficients. From Equation (4.28) we have

$$
\begin{aligned}
\phi^{\prime} & =\sum_{k=1}^{\infty} a_{k} k \alpha e^{k \alpha z} \\
\phi^{\prime \prime} & =\sum_{k=1}^{\infty} a_{k} k^{2} \alpha^{2} e^{k \alpha z} \\
8 \phi^{2} & =8\left(\sum_{k=2}^{\infty} \sum_{i=1}^{k-1} a_{k-i} a_{i} e^{k \alpha z}+x_{0}^{2}+2 x_{0} \sum_{k=1}^{\infty} a_{k} e^{k \alpha z}\right) \\
-2 \phi^{\prime 2} & =-2 \sum_{k=2}^{\infty} \sum_{i=1}^{k-1}(k-i) i \alpha^{2} a_{k-i} a_{i} e^{k \alpha z} \\
-2 \phi^{\prime \prime}\left(2 \phi-\phi^{\prime}\right)=-2 & \sum_{k=2}^{\infty} \sum_{i=1}^{k-1}(k-i)^{2}(2-i \alpha) \alpha^{2} a_{k-i} a_{i} e^{k \alpha z}-4 x_{0} \sum_{k=1}^{\infty} a_{k} k^{2} \alpha^{2} e^{k \alpha z}
\end{aligned}
$$

Substituting Equations (4.28)-(4.33) into the irreversible Equation (4.27) we have, 


$$
\begin{aligned}
-c x_{0}-8 x_{0}^{2}-g & +\sum_{k=1}^{\infty}\left((k \alpha)^{2}\left(c+4 x_{0}\right)-\left(16 x_{0}+c\right)\right) a_{k} e^{k \alpha z} \\
& =\sum_{k=2}^{\infty} \sum_{i=1}^{k-1}\left[8-2(k-i) i \alpha^{2}-2(k-i)^{2}(2-i \alpha) \alpha^{2}\right] a_{k-i} a_{i} e^{k \alpha z}
\end{aligned}
$$

where the quantity $-c x_{0}-8 x_{0}^{2}-g$ is identically zero, being $x_{0}$ an equilibrium of equation (4.27).

Now we compare the coefficients of $e^{k \alpha z}$ for each $\mathrm{k}$, for $\mathrm{k}=1$, we have from Equation (4.34),

$$
\left(\alpha^{2}\left(c+4 x_{0}\right)-\left(16 x_{0}+c\right)\right) a_{1}=0
$$

Assuming $a_{1} \neq 0$ (otherwise $a_{k}=0$ for all $k>1$ by induction), results in the two possible values of $\alpha$ :

$$
\alpha_{1}=\sqrt{\frac{16 x_{0}+c}{c+4 x_{0}}}, \quad \alpha_{2}=-\sqrt{\frac{16 x_{0}+c}{c+4 x_{0}}}
$$

As $x_{0}$ is a saddle point (here we skip all the details about the possible choice of $\mathrm{c}$ and $\mathrm{g}$, as it has been done in Chapter 3), the quantities $\alpha_{1,2}$ have opposite real parts.

As our solution (4.28) needs to converge for $z>0$, we pick the negative $\operatorname{root} \alpha=\alpha_{2}$. Thus we have

$$
\phi^{+}(z)=\sum_{k=1}^{\infty} a_{k} e^{k \alpha_{2} z}+x_{0}
$$


For $\mathrm{k}=2$, from Equation (4.34) we have

$$
F\left(2 \alpha_{2}\right) a_{2}=\left(8-6 \alpha_{2}{ }^{2}+2 \alpha_{2}{ }^{3}\right) a_{1}^{2}
$$

where $F(k \alpha)=(k \alpha)^{2}\left(c+4 x_{0}\right)-\left(16 x_{0}+c\right)$

Thus, we obtain

$$
a_{2}=\frac{\left(8-6 \alpha_{2}^{2}+2 \alpha_{2}^{3}\right) a_{1}^{2}}{F\left(2 \alpha_{2}\right)}
$$

Similarly for $k=3$ we have,

$$
a_{3}=\sum_{i=1}^{2} \frac{\left[8-2(3-i) \alpha_{2}{ }^{2}\left(-i-3 i \alpha_{2}+i^{2} \alpha_{2}+6\right)\right] a_{3-i} a_{i}}{F\left(3 \alpha_{2}\right)}
$$

Thus, for $k>2$

$$
a_{k}=\sum_{i=1}^{k-1} \frac{\left[8-2(k-i) i \alpha_{2}{ }^{2}-2(k-i)^{2}\left(2-i \alpha_{2}\right) \alpha_{2}{ }^{2}\right] a_{k-i} a_{i}}{F\left(k \alpha_{2}\right)}
$$

Therefore, the coefficients $a_{k}, k>1$ can be directly obtained by iterating the Equation (4.40) yielding:

$$
a_{k}=\varphi_{k} a_{1}^{k}
$$

where $\varphi_{k}, k>1$ are functions which can be obtained using Equations (4.38)-(4.40). They depend on $\alpha_{2}$ and the coefficients of the Equation (4.27). 
The first part of the homoclinic orbit corresponding to $z>0$ has thus been determined in terms of $a_{1}$ :

$$
\phi^{+}(z)=x_{0}+a_{1} e^{\alpha_{2} z}+\sum_{k=2}^{\infty} \varphi_{k} a_{1}^{k} e^{k \alpha_{2} z}
$$

We shall now construct the second part of the homoclinic orbit corresponding to $z<0$. Since the Equation (4.27) is not reversible, we do not have any symmetric property for the solution, therefore we impose the following solution form:

$$
\phi^{-}(z)=\sum_{k=1}^{\infty} b_{k} e^{k \alpha z}+x_{0}
$$

where real part of $\alpha>0$, because the solution $\phi^{-}(z)$ needs to converge for $z<0$. Working as for $z>0$, we obtain for $k=1$ the following equation

$$
\left((\alpha)^{2}\left(c+4 x_{0}\right)-\left(16 x_{0}+c\right)\right) b_{1}=0
$$

Assuming $b_{1} \neq 0$ (otherwise $b_{k}=0$ for all $k>1$ by induction), the Equation (4.44) is solved by choosing $\alpha=\alpha_{1}$. Moreover, for $k>1$ one obtains the following equation

$$
\begin{aligned}
F\left(k \alpha_{1}\right) b_{k}=\sum_{i=1}^{k-1}\left[8-2(k-i) i \alpha_{1}^{2}-2(k-i)^{2}\left(2-i \alpha_{1}\right) \alpha_{1}^{2}\right] b_{k-i} b_{i} \\
b_{k}=\sum_{i=1}^{k-1} \frac{\left[8-2(k-i) i \alpha_{1}^{2}-2(k-i)^{2}\left(2-i \alpha_{1}\right) \alpha_{1}^{2}\right] b_{k-i} b_{i}}{F\left(k \alpha_{1}\right)}
\end{aligned}
$$

where the polynomial $F\left(k \alpha_{1}\right)=\left((k \alpha)^{2}\left(c+4 x_{0}\right)-\left(16 x_{0}+c\right)\right)$ is non-zero for $k>1$. Therefore, the series coefficients can be easily obtained as for $z<0$ as follows: 


$$
b_{k}=\psi_{k} b_{1}^{k}
$$

where $\psi_{k}$ are given in terms of $b_{1}$ and the coefficient of Equation (4.27). As we want to construct a solution of the following form:

$$
\phi(z)= \begin{cases}\phi^{+}(z)+x_{0} & z>0 \\ 0 & z=0 \\ \phi^{-}(z)+x_{0} & z<0\end{cases}
$$

which is continuous at $z=0$, we impose

$$
\begin{aligned}
& \phi^{+}(0)=a_{1}+\sum_{k=2}^{\infty} \varphi_{k} a_{1}^{k}+x_{0}=0 \\
& \phi^{-}(0)=b_{1}+\sum_{k=2}^{\infty} \psi_{k} b_{1}^{k}+x_{0}=0
\end{aligned}
$$

hence we choose $a_{1}$ and $b_{1}$ as the nontrivial solutions of the above polynomial equations (4.49) and (4.50).

Let us consider the first numerical test. We choose $\mathrm{c}=-1$ and $\mathrm{g}=-1.25$. With this choice both the equilibria $\mathrm{z}_{1}=0.4627$ and $\mathrm{z}_{2}=-0.3377$ are saddles, but only a homoclinic orbit to the point $z_{1}$ has been observed (See Figure 6(e)). Here we construct this orbit. The eigenvalues relative to the point $z_{1}$ are $\alpha_{1}=-2.7434$ and $\alpha_{2}=2.7434$. If we truncate the series solution at $\mathrm{M}=25$, the continuity equation (4.49) has the only solution $\mathrm{a}_{1}=-0.06$ and the continuity equation (4.50) has the only solution $b_{1}=-0.3948$. 

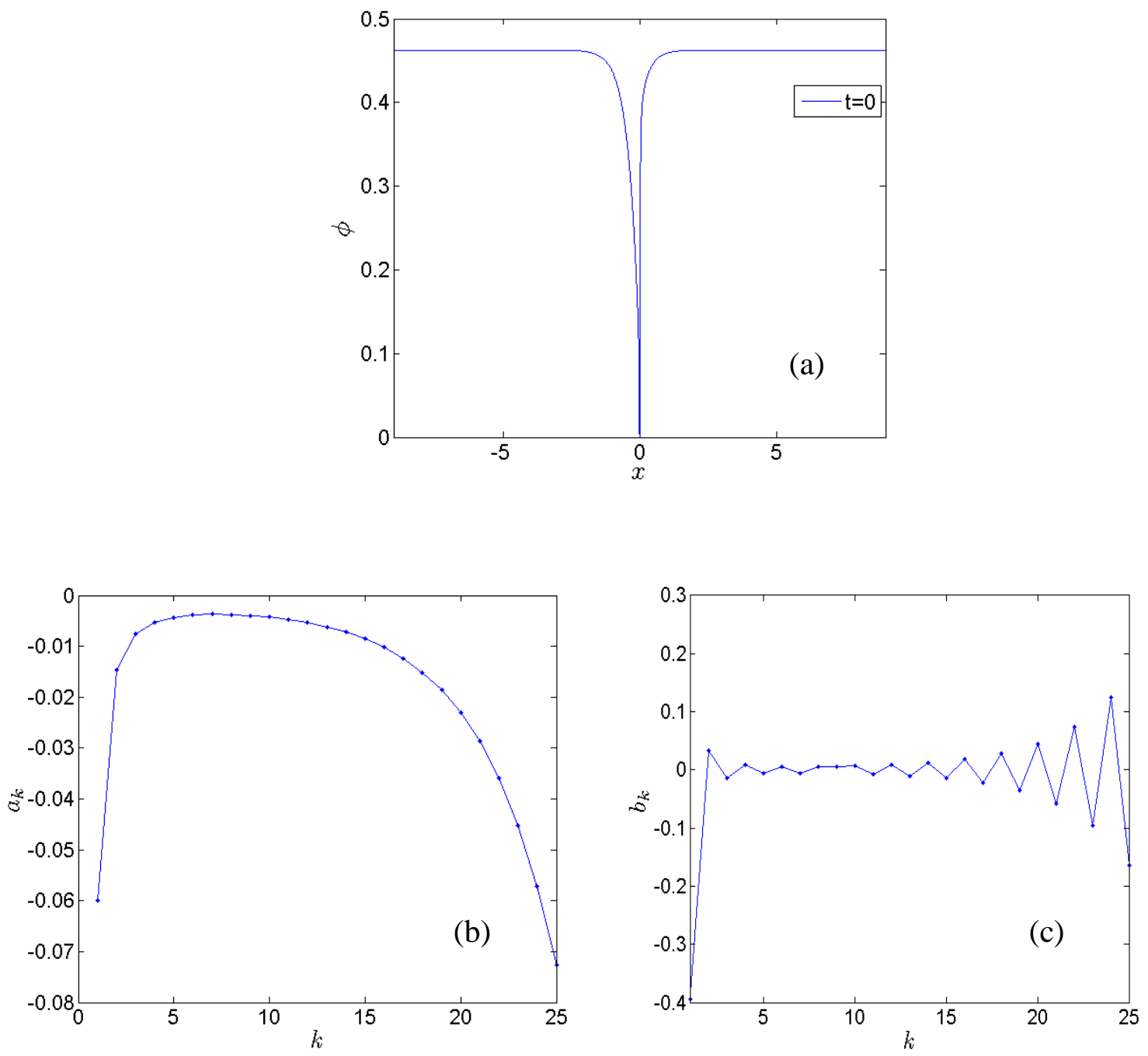

Figure 13: The parameters are chosen as $c=-1$ and $g=-1.25$. (a) Series solution for the homoclinic orbit to the point $x_{0}=0.4627$. (b) The series coefficients $a_{k}$ versus $\mathrm{k}$ does not converge. (c) The series coefficients $b_{k}$ versus $\mathrm{k}$ does not converge. 
Choosing this value for $a_{1}$ and $b_{1}$ we obtain the continuous solution as in Figure 13(a). Nevertheless, the corresponding series coefficients $a_{k}$ and $b_{k}$ both diverge, as shown in Figures 13(b)-(c).

To obtain the convergence of the series coefficients $a_{k}$ we arbitrarily choose $a_{1}=0.04$, see Figure 14(b). With this choice of $a_{1}$ the solution (4.48) becomes discontinuous at the origin. Therefore, we choose $b_{1}=0.0348$ in such a way that $\phi^{-}(0)=\phi^{+}(0)=0.4977$ and the solution is still continuous into the origin. This value for $b_{1}$ also preserves the convergence of the series coefficients $b_{k}$, see Figure 14(c). The travelling wave nature of the obtained continuous solution has been shown in Figure 14(a). As it is obvious that the solution is similar to a symmetric peakon having $\alpha_{1}=-2.7434$ and $\alpha_{2}=2.7434$.

Let us consider a second numerical test choosing $\mathrm{c}=3$ and $\mathrm{g}=0.1$. In this case the equilibrium $\mathrm{z}_{1}=-0.0370$ is a saddle and $\mathrm{z}_{2}=-0.3380$ is a center. Here we construct the homoclinic orbit to the point $\mathrm{z}_{1}$ as observed in Chapter 3 [Figure 6(a)].

The eigenvalues relative to the point $\mathrm{z}_{1}$ are $\alpha_{1}=-0.9189$ and $\alpha_{2}=0.9189$. We truncate the series solution at $M=10$, the continuity equation (4.49) has the only solution $\mathrm{a}_{1}=-0.0368$ and the continuity equation (4.50) has the only solution $b_{1}=-0.0362$. Choosing theses values for $a_{1}$ and $b_{1}$ we obtain the continuous solution as in Figure 15(a). Moreover, the corresponding series coefficients $a_{k}$ and $b_{k}$ both converge, as shown in Figures 15(b)-(c). As it is obvious the solution is similar to a symmetric peakon having $\alpha_{1}=-0.9189$ and $\alpha_{2}=0.9189$. The traveling wave nature of the solution is shown in Figure 15(a) for different time instant. 


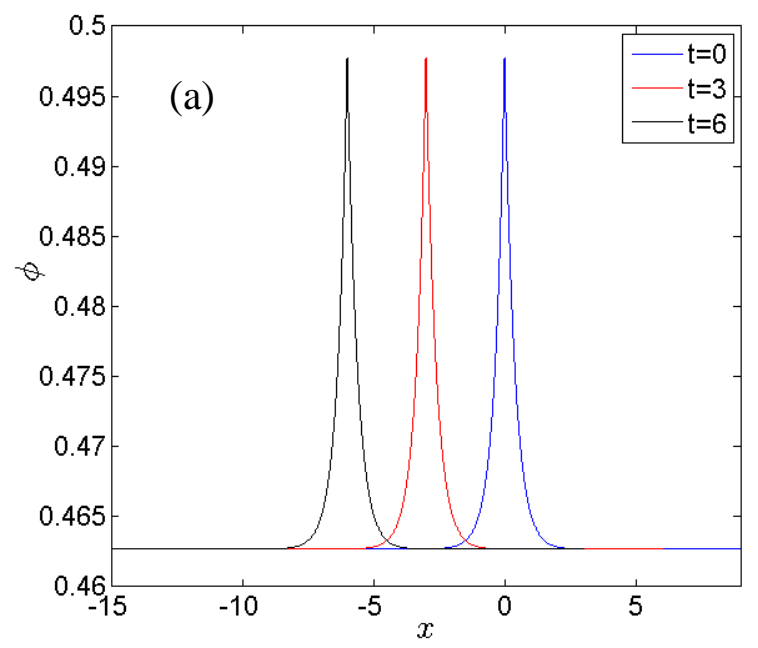

(b)

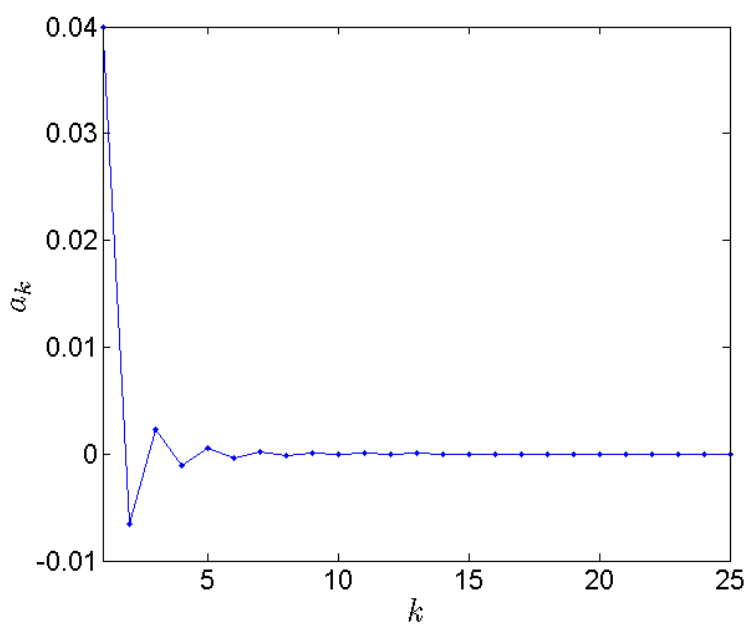

(c)

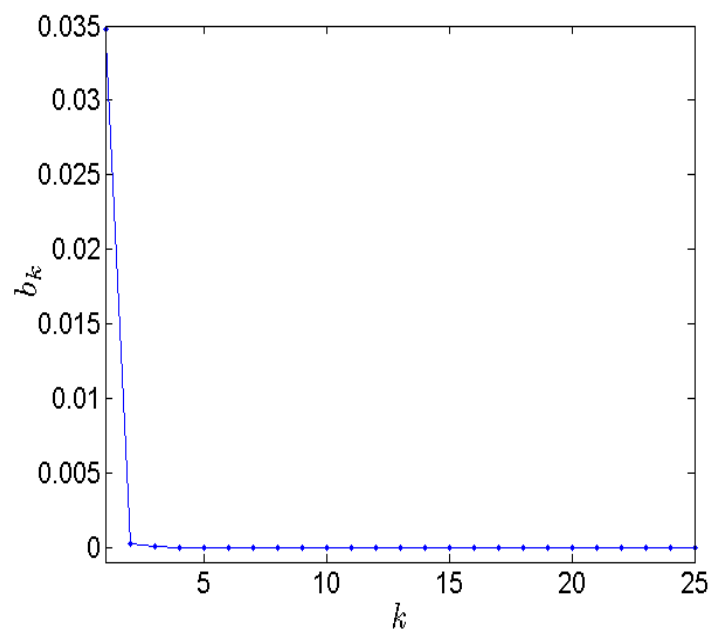

Figure 14: The parameters are chosen as $c=-1$ and $g=-1.25$. (a) Series solution for the homoclinic orbit to the point $x_{0}=0.4627$. Here, $a_{1}=0.04$ and $b_{1}=0.0348$ to have continuity a the origin: $\phi^{+}(0)=\phi^{-}(0)=0.4977$. (b) The series coefficients $a_{k}$ versus k converges. (c) The series coefficients $b_{k}$ versus k converges. 
(a)

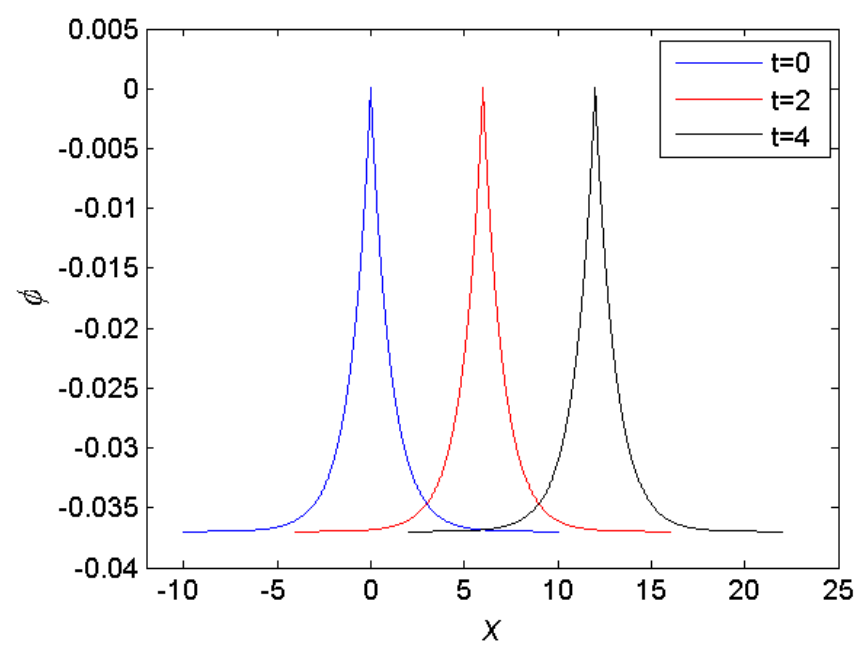

(b)

(c)
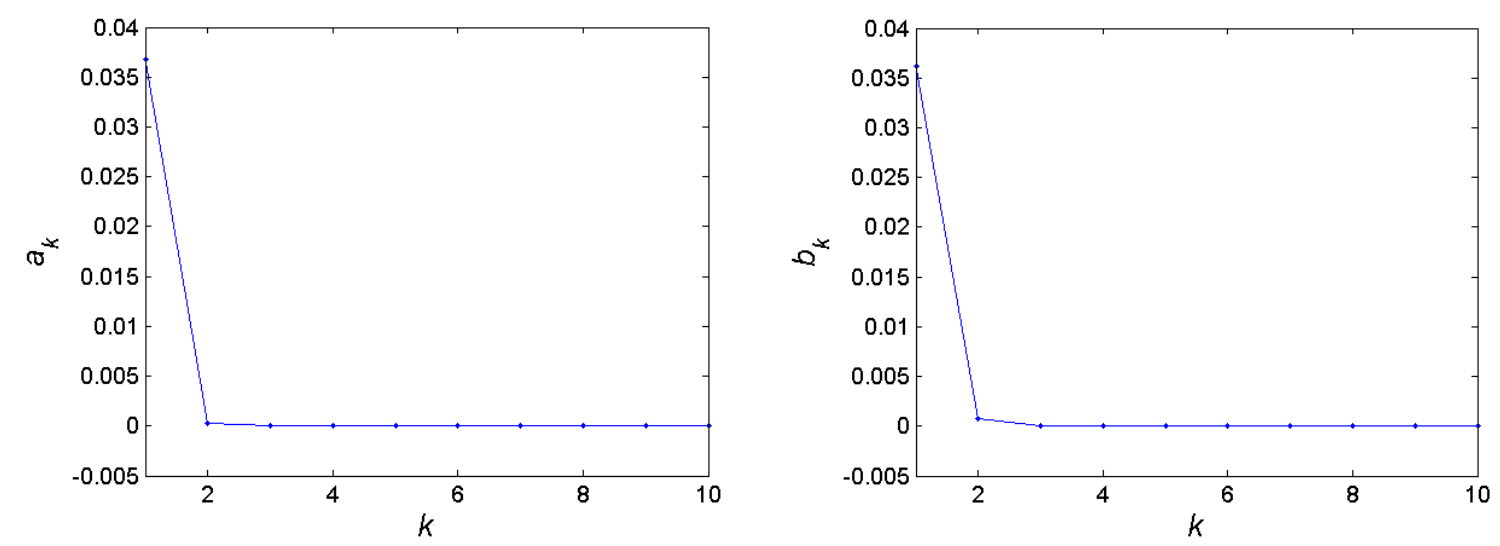

Figure 15: The parameters are chosen as $c=3$ and $g=0.1$ (a) Series solution for the homoclinic orbit to the point $x_{0}=-0.0370$. (b) The series coefficients $a_{k}$ versus k rapidly converges. (c) The series coefficients $b_{k}$ versus k rapidly converges. 
Notice the "symmetry" properties of the solution just changing the sign of c, as the equilibria $\mathrm{z}_{1}$ and $\mathrm{z}_{2}$ exchange their role. Choosing $\mathrm{c}=1$ and $\mathrm{g}=-1.25$, the equilibria $\mathrm{z}_{1}=0.3377$ and $\mathrm{z}_{2}=-0.4627$ are both saddles, but only a homoclinic orbit to the point $\mathrm{z}_{2}$ has been observed (See Figure 6(c)). The eigenvalues relative to the point $\mathrm{z}_{2}$ are $\alpha_{1}=-2.7434$ and $\alpha_{2}=2.7434$. If we truncate the series solution at $M=25$, the continuity equation (4.49) has the only solution $\mathrm{a}_{1}=0.06$ and the continuity equation (4.50) has the only solution $b_{1}=0.3948$. Choosing these values for $a_{1}$ and $\mathrm{b}_{1}$ we obtain a continuous solution, but the corresponding series coefficients $a_{k}$ and $b_{k}$ both diverge, as already found in the "symmetric" case shown in Figures 13(b)-(c).

To obtain the convergence of the series coefficients $a_{k}$ we arbitrarily choose $a_{1}=0.04$. With this choice of $a_{1}$ the solution (4.48) becomes discontinuous at the origin. Therefore, we choose $b_{1}=0.0521$ in such a way that $\phi^{-}(0)=\phi^{+}(0)=-0.4112$ and the solution is still continuous into the origin. This value for $b_{1}$ also preserves the convergence of the series coefficients $b_{k}$, see Figure 16(c). The travelling nature of the obtained continuous solution has been shown in Figure 16(a). As it is obvious that the solution is similar to a symmetric peakon having $\alpha_{1}=-2.7434$ and $\alpha_{2}=2.7434$.

Next, we discuss the behavior of solution for a particular case when $g=0$. When $g=0$, Equation (3.8) has two fixed points, $(0,0)$ which is a saddle (it corresponds to the equilibrium point $\left.\mathrm{z}_{1}\right)$ and $\left(-\frac{1}{8} c, 0\right)$, which is a center. Let us compute the series solution of the homoclinic orbit to the origin (See Figure 6(f), 6(g)), i.e. $x_{0}=0$ in Equations (4.28), (4.48)-(4.50). Interestingly, we have noticed that all the series coefficients $a_{k}$ in Equation (4.28) become zero for all values of $k$, independently on the choice of $a_{1}$. Therefore, an arbitrary value of $a_{1}$ is taken for all the plots when $\mathrm{g}=0$. 
(a)

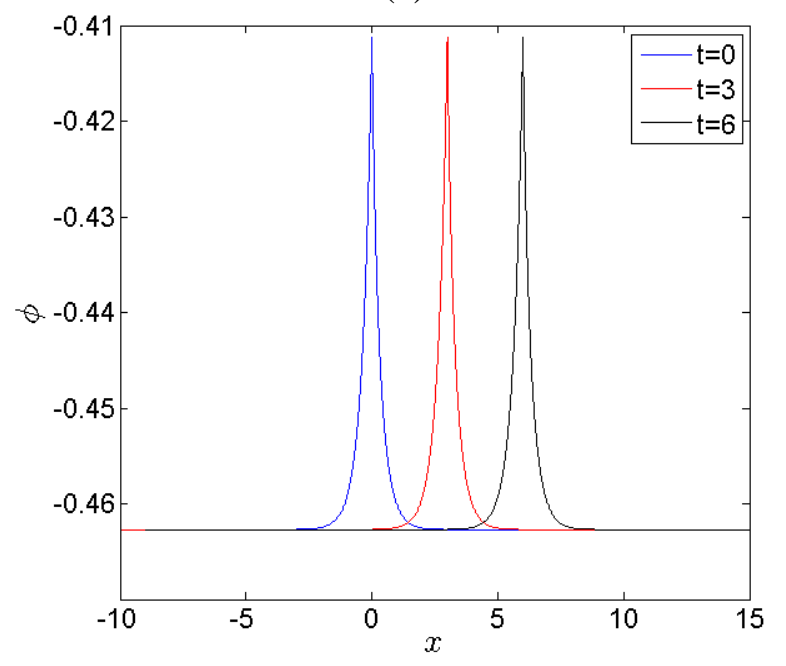

(b)

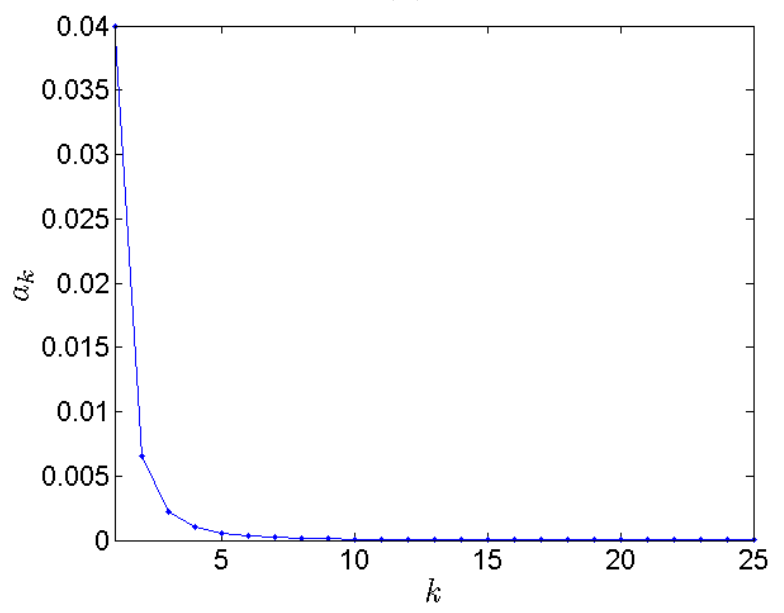

(c)

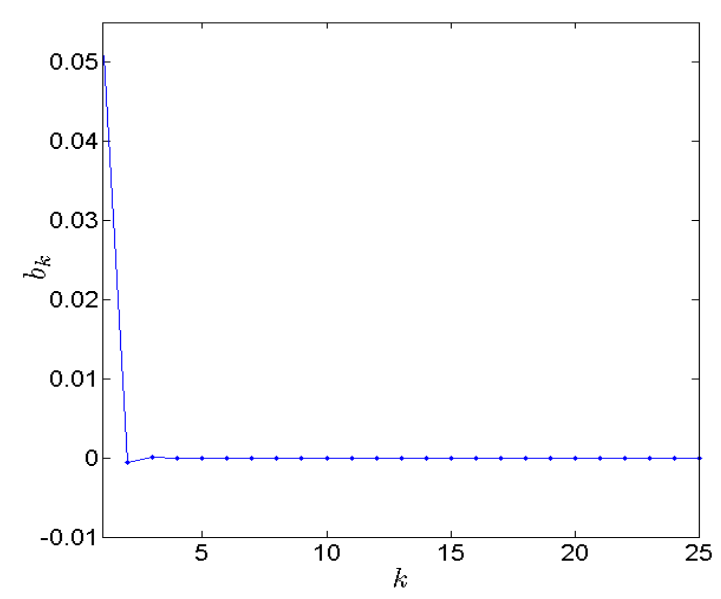

Figure 16: The parameters are chosen as $c=1$ and $g=-1.25$. (a) Series solution for the homoclinic orbit to the point $x_{0}=-0.4627$. Here $a_{1}=0.04$ and $b_{1}=0.0521$ to have continuity a the origin $\phi^{+}(0)=\phi^{-}(0)=-0.4112$. (b) The series coefficients $a_{k}$ versus $\mathrm{k}$ converges. (c) The series coefficients $b_{k}$ versus k converges. 
Note that the zero solution resulting for $\mathrm{z}>0$ is worth for future investigation as to whether it is the only possible homoclinic solution in that domain. It is also reminiscent of the phenomenon of 'Quenching' in nonlinear oscillators where the solutions go to zero in certain parts of the domain.

For $\mathrm{z} \leq 0, \mathrm{~b}_{\mathrm{k}}$ is zero for $\mathrm{k}>2$. Therefore, to obtain $\phi(0)=0$, from Equation (4.50) we get $b_{1}+b_{2}=0$, where, $b_{2}=\left(4 b_{1}^{2}\right) /(3 c)$. So, to satisfy the continuity as shown in Equation (4.48) the value of $b_{1}$ will be, $b_{1}=\frac{3 c}{4}=-b_{2}$. We also get another solution $b_{1}=0$ from Equation (4.48) which obviously will not yield any series solution. However in present case for $\mathrm{g}=0$, since the right side of the solution does not have any $a_{1}$ that satisfies Equation (4.48) we took $b_{1}$ so that $\phi^{-}(0)=\phi^{+}(0)$.

In Figure 17(a), [using Equation (4.43) and relation $z=x-c t$ ] $\phi(x)$ is plotted against $x$ for $\mathrm{c}=0.5,1.0$, and 2.0 . The initial value was arbitrarily taken as $\mathrm{a}_{1}=0.1$ and as mentioned before all other $a_{k}($ for $k>2)$ are zero. Therefore, the initial value was $\phi^{+}(0)=0.1$. For a particular value of c, to maintain $\phi^{-}(0)=\phi^{+}(0)$, we obtain two different solutions of $b_{1}$. For example, the solutions for $b_{1}$ are for $c=0.5, b_{1}=-0.4570$ and $0.0821 ;$ for $c=1.0, b_{1}=-0.8393$ and $0.0894 ;$ and for $c=2.0$ $b_{1}=-1.5940$ and 0.0941 . For negative values of $b_{1}$ as seen in Figure 17(a), the negative side is more susceptible to variation of $c$, which is not seen in Figure 17(c) for positive values of $b_{1}$. From Figures 17(a) and 17(c) we can see that, only one set of $b_{1}$ (here the positive values) yield a peakon shaped wave profile; whereas for the negative values of $b_{1}$, we have profile for M-shaped wave in negative side but a peakon shaped profile on the positive side [see Figure 17(a)]. 

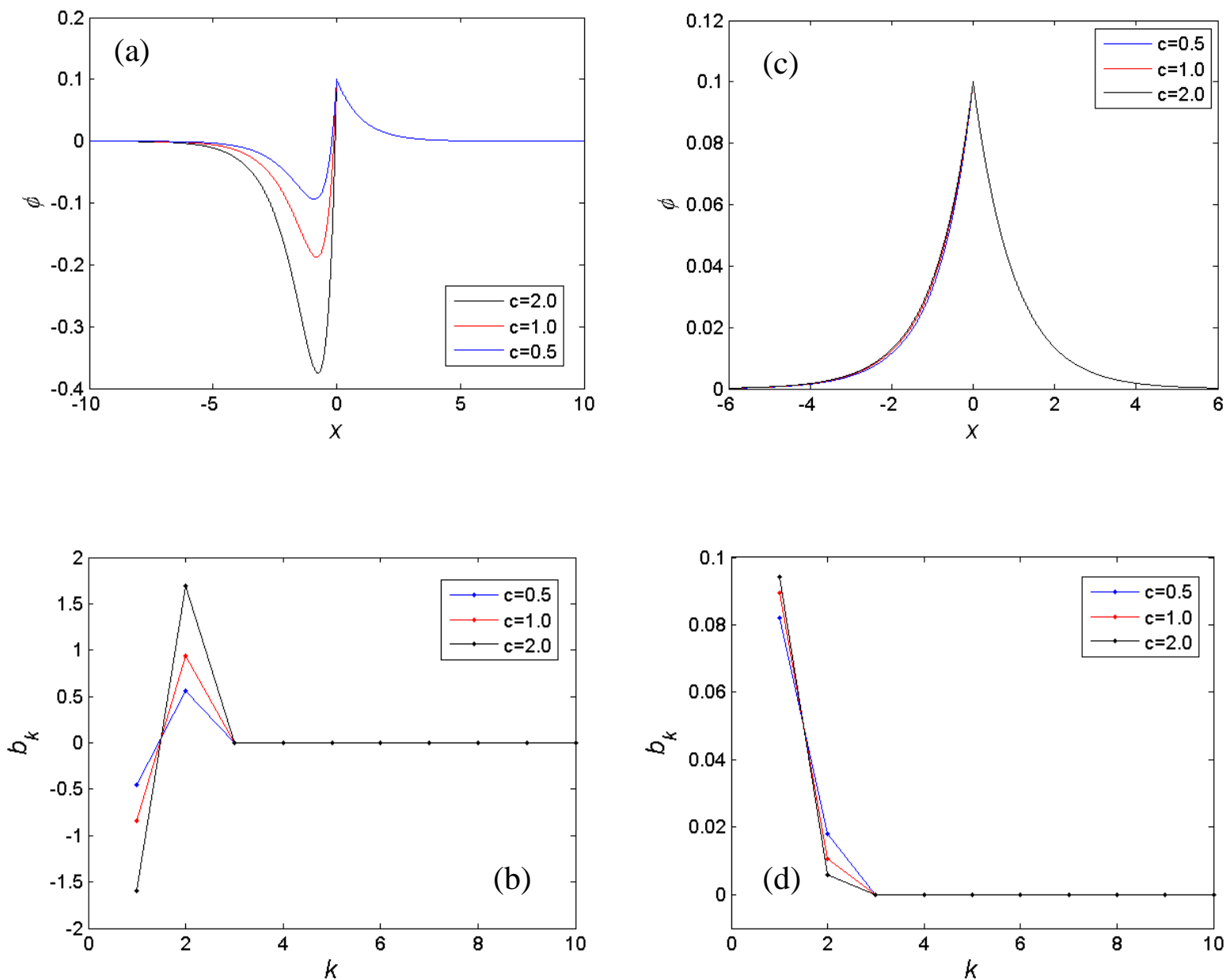

Figure 17: $\phi(x)$ plotted as a function of $x$ for $\mathrm{c}=0.5,1.0$, and 2.0 for (a) negative, and (c) positive values of $\mathrm{b}_{1}$. The series coefficients $b_{k}$ verses $k$ is plotted for $\mathrm{c}=0.5,1.0$ and 2.0 for (b) negative, and (d) positive values of $b_{1}$.

Figures 17(b) and 17(d) show the plot of $b_{k}$ versus $k$ for negative and positive values of $b_{1}$, where it is obvious that $b_{k}$ is zero when $\mathrm{k}$ is greater than 2 ; therefore only two values of $b_{k}$ is available. 

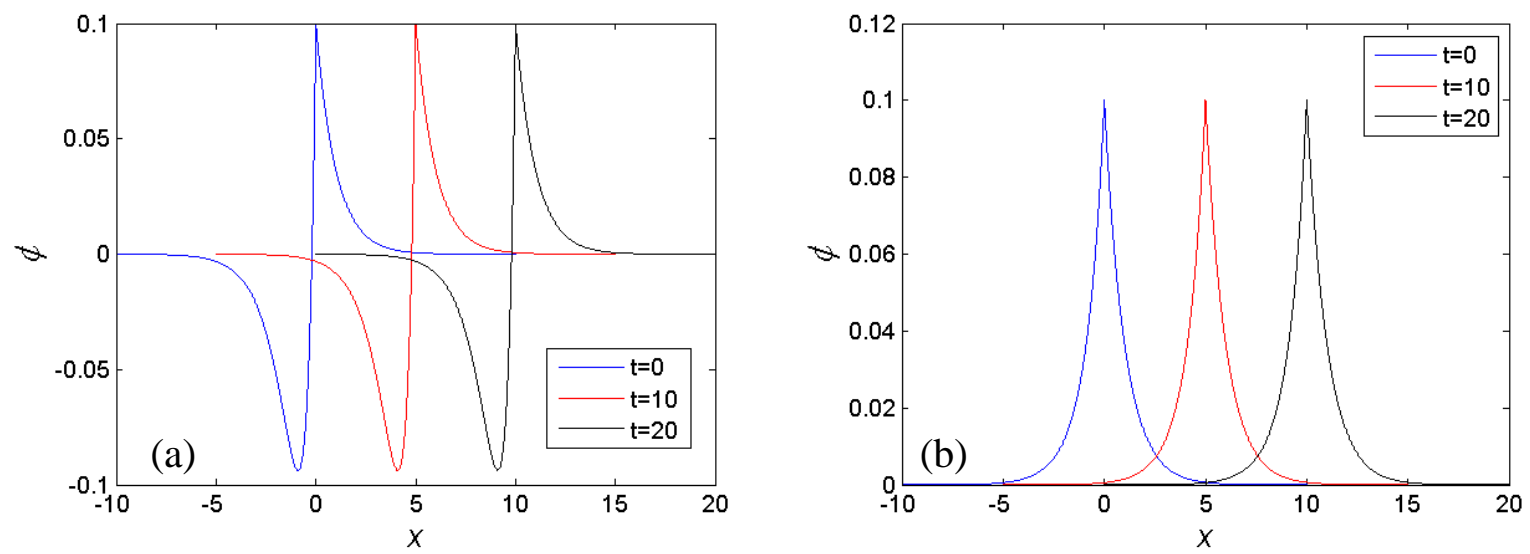

Figure 18: $\phi(x)$ plotted as a function of $\mathrm{x}$ for $\mathrm{c}=0.5$ at different time instances showing wave nature of the solution for (a) $b_{1}=-0.4570$, and (b) $b_{1}=0.0821$.

To demonstrate the traveling wave nature of the solution $\phi(x)$, Figure 18 shows the plots of $\phi(\mathrm{x})$ at constant times, $\mathrm{t}=0,10$, and 20 . As mentioned before, for $\mathrm{c}=0.5$ we have two solutions of $b_{1}$ to maintain continuity of $\phi^{-}(0)=\phi^{+}(0)=0.1$ which are $b_{1}=-0.4570$ and 0.0821 .

\subsection{Infinite Series for homoclinic orbits of Equation (3.12)}

In the particular case $g=0$, Equation (3.12) can be rewritten as:

$$
\left(c-\phi^{2}+\phi^{\prime 2}\right)\left(\phi^{\prime \prime}-\phi\right)=0
$$

where the ' indicates the derivative with respect to $z$ and it can be exactly integrated. The solutions are:

$$
\phi_{1}(z)=k_{1} e^{z}+k_{2} e^{-z}
$$




$$
\begin{aligned}
& \phi_{2}(z)=\frac{1}{4} e^{\left(-z-k_{3}\right)}\left[4 \operatorname{exexp}(2 z)+\exp \left(2 k_{4}\right)\right] \\
& \phi_{3}(z)=\frac{1}{4} e^{\left(-z-k_{5}\right)}\left[4 e+\exp \left(2 z+2 k_{6}\right)\right]
\end{aligned}
$$

for all $k_{i} \in \mathbb{R}, \quad i=1, \cdots \cdots 6$.

In the general case with $g$ different from zero, let us consider an equilibrium point $x_{0}$ of Equation (3.14) and assume to choose the parameters $\mathrm{c}$ and $\mathrm{g}$ in such a way that it is a saddle point and a homoclinic orbit to this equilibrium is given (As done in the stability analysis in Chapter 3). Equation (3.12) can be written as

$$
-c \phi+c \phi^{\prime \prime}=\phi^{2} \phi^{\prime \prime}+\phi \phi^{\prime 2}-\phi^{\prime 2} \phi^{\prime \prime}-\phi^{3}-g
$$

Suppose for $z>0$

$$
\phi^{+}(z)=\sum_{k=1}^{\infty} a_{k} e^{k \alpha z}+x_{0}
$$

where $\alpha<0$ is an undetermined constant and, $a_{k}, k \geq 1$ are, at the outset, arbitrary coefficients. Substituting the series (4.53) into the Equation (4.52), we obtain the following expressions for each term:

$$
c \phi^{\prime \prime}=c \sum_{k=1}^{\infty} a_{k} k^{2} \alpha^{2} e^{k \alpha z}
$$




$$
\begin{gathered}
\phi^{2} \phi^{\prime \prime}=\sum_{k=3}^{\infty} \sum_{j=2}^{k-1} \sum_{l=1}^{j-1} a_{l} a_{j-l} a_{k-j}(k-j)^{2} \alpha^{2} e^{k \alpha z}+2 x_{0} \sum_{k=2}^{\infty} \sum_{j=1}^{k-1}(k-j)^{2} \alpha^{2} a_{k-j} a_{j} e^{k \alpha z} \\
\quad+x_{0}^{2} \sum_{k=1}^{\infty} a_{k} k^{2} \alpha^{2} e^{k \alpha z} \\
\phi \phi^{\prime 2}=\sum_{k=3}^{\infty} \sum_{j=2}^{k-1} \sum_{l=1}^{j-1} a_{l} a_{j-l} a_{k-j}(j-l) l \alpha^{2} e^{k \alpha z} \\
\quad+x_{0} \sum_{k=2}^{\infty} \sum_{j=1}^{k-1}(k-j) a_{k-i} a_{j} j \alpha^{2} e^{k \alpha z} \\
\phi^{\prime 2} \phi^{\prime \prime}=\sum_{k=3}^{\infty} \sum_{j=2}^{k-1} \sum_{l=1}^{j-1} a_{l} a_{j-l} a_{k-j}(j-l) l(k-j)^{2} \alpha^{4} e^{k \alpha z} \\
\phi^{3}=\sum_{k=3}^{\infty} \sum_{j=2}^{k-1} \sum_{l=1}^{j-1} a_{l} a_{j-l} a_{k-j} e^{k \alpha z}+3 x_{0} \sum_{k=2}^{\infty} \sum_{j=1}^{k-1} a_{k-j} a_{i} e^{k \alpha z}+3 x_{0}^{2} \sum_{k=1}^{\infty} a_{k} e^{k \alpha z}+x_{0}^{3}
\end{gathered}
$$

Using Equations (4.53)-(4.58) in the reversible Equation (4.52) we have

$$
\begin{aligned}
-c x_{0}+x_{0}^{3}+ & g \\
& +\sum_{k=1}^{\infty}\left[(k \alpha)^{2}\left(c-x_{0}^{2}\right)+3 x_{0}^{2}-c\right] a_{k} e^{k \alpha z} \\
& +\sum_{k=3}^{\infty} \sum_{j=2}^{k-1} \sum_{l=1}^{j-1}\left[-(k-j)^{2} \alpha^{2}-(j-l) l \alpha^{2}+l(j-l)(k-j)^{2} \alpha^{4}+1\right] a_{l} a_{j-l} a_{k-j} \\
& +\sum_{k=2}^{\infty} \sum_{j=1}^{k-1}\left[-2(k-j)^{2} \alpha^{2}-(k-j) j \alpha^{2}+3\right] x_{0} a_{k-j} a_{j} e^{k \alpha z}=0
\end{aligned}
$$


As $x_{0}$ is an equilibrium for the equation (3.14), $-c x_{0}+x_{0}^{3}+g=0$. Comparing the coefficients of $e^{k \alpha z}$ for each $k$, one has for $\mathrm{k}=1$,

$$
\left[\alpha^{2}\left(c-x_{0}^{2}\right)+3 x_{0}^{2}-c\right] a_{1}=0 .
$$

Assuming $a_{1} \neq 0$ (otherwise $a_{k}=0$ for all $k>1$ by induction), results in the two possible values of $\alpha$ :

$$
\alpha_{1,2}= \pm \sqrt{\frac{3 x_{0}^{2}-c}{x_{0}^{2}-c}}
$$

We are dealing with the case when the equilibrium $x_{0}$ is a saddle, i.e. when $\mathrm{c}<x_{0}^{2}$ or c $>3 x_{0}^{2}$. In this case, as our series solution for Equation (4.53) needs to converge for $z>0$, we pick the negative root $\alpha_{2}=-\sqrt{\frac{3 x_{0}^{2}-c}{x_{0}^{2}-c}}$, therefore:

$$
\phi^{+}(z)=\sum_{k=1}^{\infty} a_{k} e^{k \alpha_{2} z}+x_{0}
$$

For k $=2$ we obtain: $\quad F\left(2 \alpha_{2}\right) a_{2}=3 x_{0}\left(1-\alpha_{2}^{2}\right) a_{1}^{2}$,

where $F(k \alpha)=(k \alpha)^{2}\left(c-x_{0}^{2}\right)+3 x_{0}^{2}-c$ and the coefficient $a_{2}$ is easily obtained in terms of $a_{1}$ :

$$
a_{2}=\frac{3 x_{0}\left(1-\alpha_{2}^{2}\right)}{F\left(2 \alpha_{2}\right)} a_{1}^{2}
$$

For k=3 we obtain:

$$
F\left(3 \alpha_{2}\right) a_{3}=2 x_{0}\left(-7 \alpha_{2}^{2}+3\right) a_{1} a_{2}+\left(\alpha^{2}-1\right)^{2} a_{1}^{3}
$$


Once substituted the formula (4.63) into the Equation (4.64) one obtains $a_{3}$ in terms of $a_{1}$. And so on for all $\mathrm{k}$ the series coefficients $a_{k}$ can be iteratively computed in terms of $a_{1}$.

Notice that the equation (3.12) is reversible, therefore the series solution for $\mathrm{z}<0$ can be easily obtained based on the intrinsic symmetry property of the equation, i.e.,

$$
\phi^{-}(z)=-\sum_{k=1}^{\infty} a_{k} e^{k \alpha_{1} z}+x_{0}
$$

We want to construct a solution of the form:

$$
\phi(z)= \begin{cases}\phi^{+}(z) & z>0 \\ 0 & z=0 \\ \phi^{-}(z) & z<0\end{cases}
$$

which is continuous at $z=0$, we impose

$$
\phi^{+}(0)=x_{0}+a_{1}+\sum_{k=2}^{\infty} \varphi_{k} a_{1}^{k}=0
$$

Hence, we choose $a_{1}$ as the nontrivial solutions of the following polynomial equations:

$$
x_{0}+a_{1}+\sum_{k=2}^{\infty} \varphi_{k} a_{1}^{k}=0
$$

In practice the Equation (4.67) is numerically solved and the corresponding series solutions are not unique. 

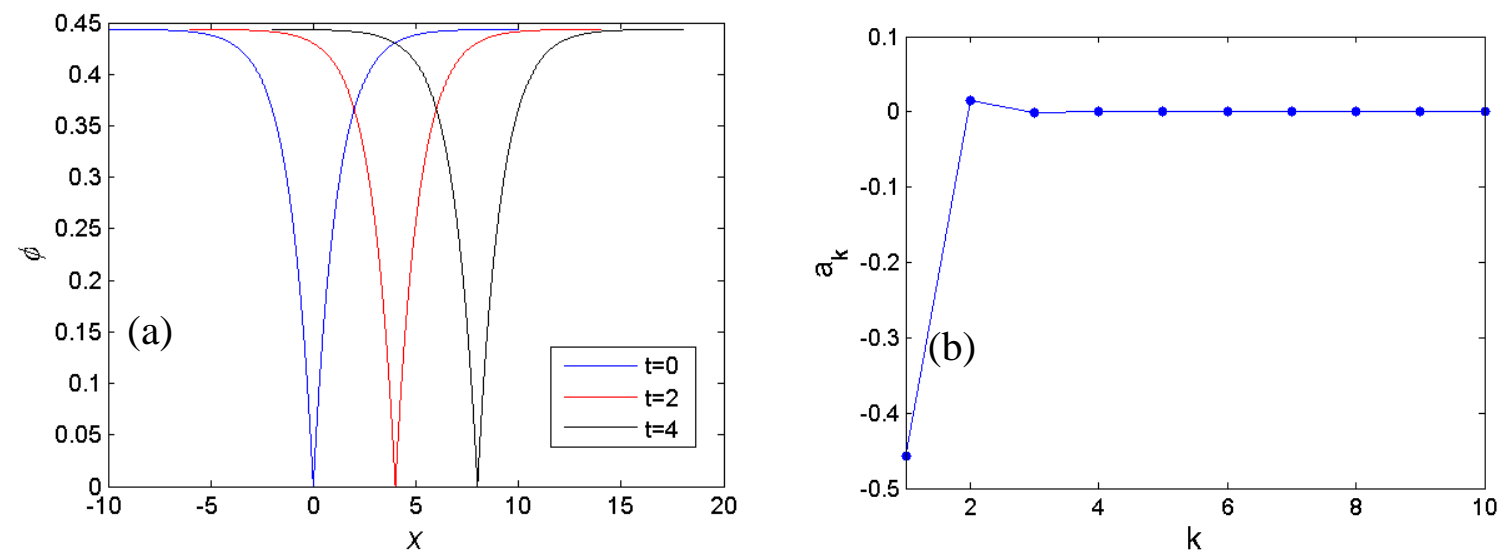

Figure 19: The parameters are chosen as $\mathrm{c}=2$ and $\mathrm{g}=0.8$, (a) Homoclinic orbit to the saddle point $z_{1}=(0.4436,0), x_{0}=0.4436$ (b) $a_{\mathrm{k}}$ versus $\mathrm{k}$, shows the series coefficients converge.

Let us now choose $\mathrm{c}=2$ and $\mathrm{g}=.8$. In this case the equation (3.14) admits three real equilibrium points, two equilibria $z_{1}=(.4436,0)$ and $z_{2}=(-1.5828,0)$ are saddles and the other $z_{3}=(1.1391,0)$ is a center. We do not observe any homoclinic orbit at the saddle point $z_{2}=(-1.5828,0)$ in the phase portrait drawn in chapter 3 . Thus we build homoclinic orbit the saddle point $z_{1}=(.4436,0)$ (See Figure $7(b)$ ). Truncating the series at $M=25$, we find that the continuity condition (4.66) is satisfied only for $\mathrm{a}_{1}=-.4572$. This choice of $\mathrm{a}_{1}$ guarantees the continuity (see Figure 19(a)), the series coefficients $a_{k}$ also converge, see Figure 19(b).

Let us now choose $\mathrm{c}=0.5$ and $\mathrm{g}=0.13$. In this case the equation (3.14) admits three real equilibrium points, two equilibria $z_{1}=(-0.8124,0)$ and $z_{2}=(0.3356,0)$ are saddles and the other $z_{3}=(0.4768,0)$ is a center. From Figure 7 , we do not observe any homoclinic orbit at the saddle point $z_{1}=(-0.8124,0)$, thus we construct the homoclinic orbit to the saddle $z_{2}=$ 
0.3356, we find that the continuity condition (4.66) does not admit any real solution. Therefore the solution will be discontinuous at the origin, or we have to choose an arbitrary $\mathrm{a}_{1}$ and impose that in zero the solution does not take the value zero, but the common value of $\phi^{+}(0)$ and $\phi^{-}(0)$ for that $\mathrm{a}_{1}$.

In Figure 20 we have chosen $a_{1}=0.1$ and $\phi(0)=0.44705$ and the travelling nature of the solution has been shown in Figure 20(a). Moreover, for this choice of the parameters the series solution converges, as shown in the following Figure 20(b) where the $a_{k}$ rapidly goes to zero.

Let us now choose $\mathrm{c}=0.5$ and $\mathrm{g}=-0.1$. In this case the equation (3.14) admits three real equilibrium points, two equilibria $z_{1}=(0.7914,0)$ and $z_{2}=(-0.2218,0)$ are saddles and the other $z_{3}=(-0.5696,0)$ is a center. From Figure 7, we do not observe any homoclinic orbit at the saddle point $z_{1}=(0.7914,0)$, thus we construct the homoclinic orbit to the saddle $z_{2}=$ -0.2218 (See Figure 7(e)), we find that the continuity condition (4.66) admits real solution for $a_{1}=0.2287$. The values of $a_{k}$ also converges to zero. In Figure 21 we have chosen $\mathrm{a}_{1}=0.2287$ and the travelling nature of the solution has been shown in Figure 21(a). Moreover, for this choice of the parameters the series solution converges, as shown in the following Figure 21(b) where the $a_{k}$ rapidly goes to zero. 

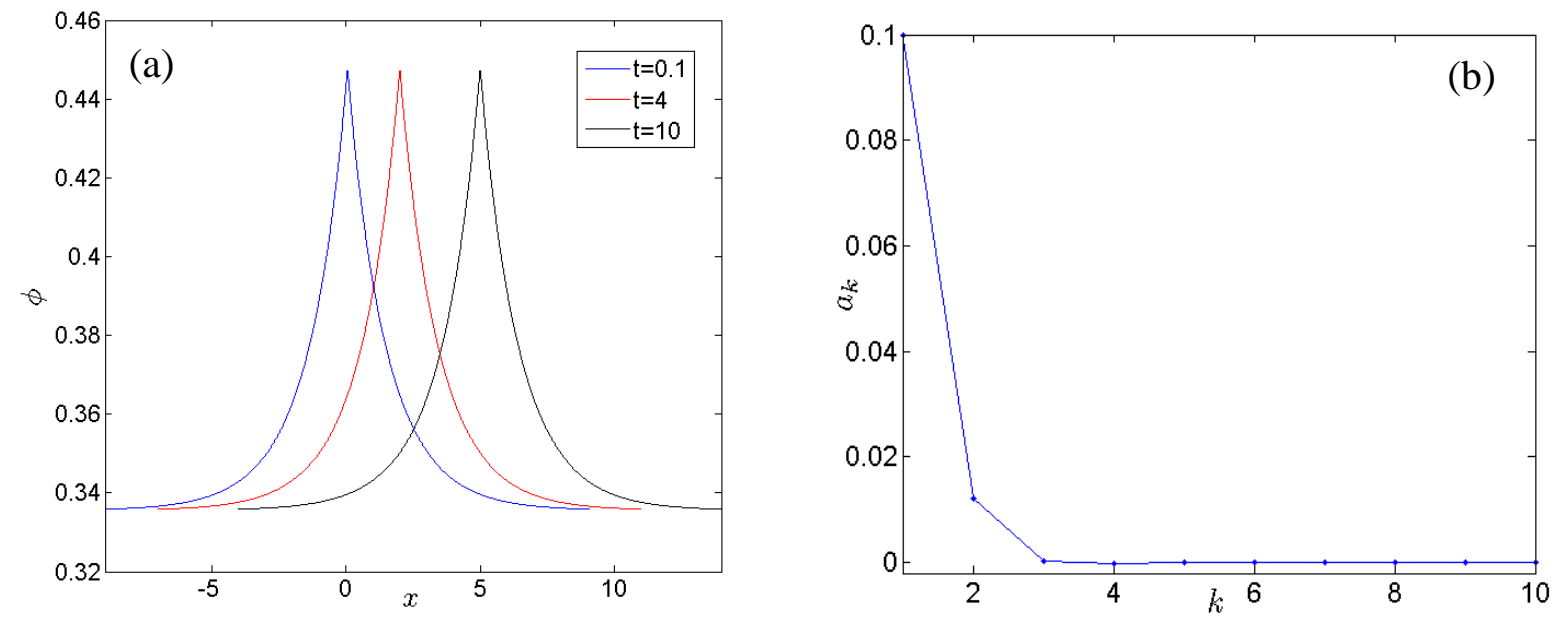

Figure 20: The parameters are chosen as $\mathrm{c}=0.5$ and $\mathrm{g}=0.13$, (a) Homoclinic orbit to the saddle point $z_{2}=0.3356$. Here $\phi(0)=0.44705$, (b) $a_{\mathrm{k}}$ versus $\mathrm{k}$, shows the series coefficients converges.
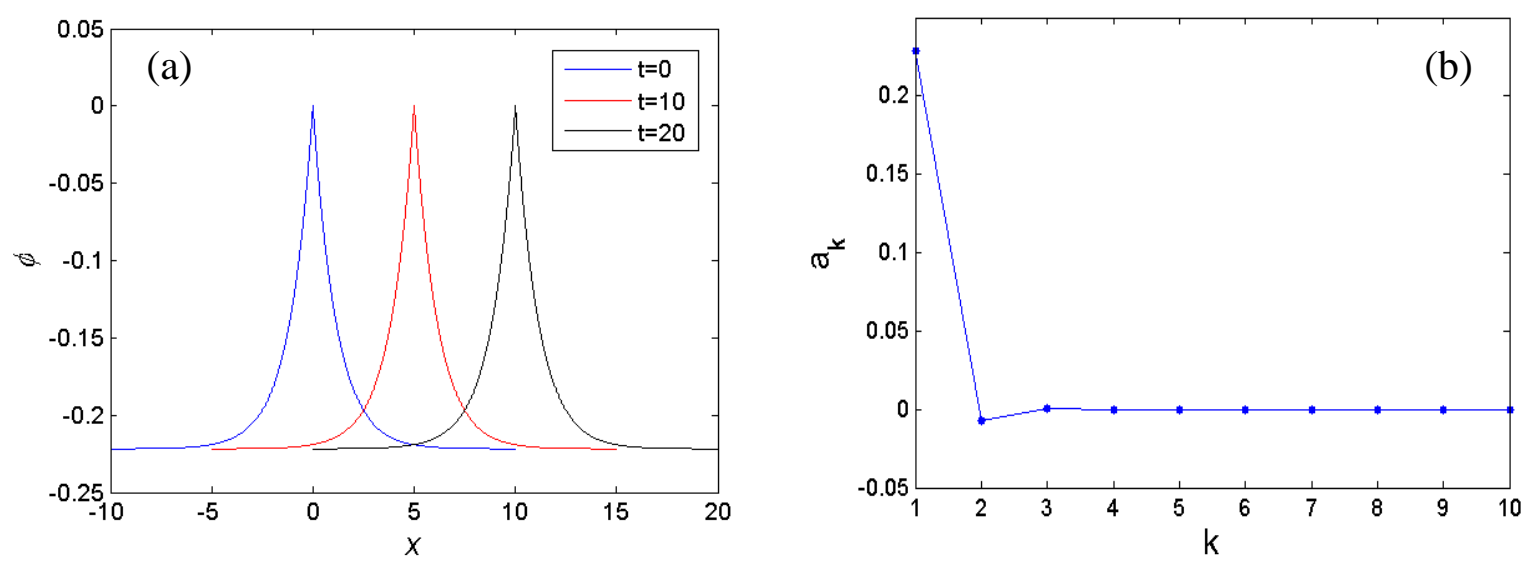

Figure 21: The parameters are chosen as $\mathrm{c}=0.5$ and $\mathrm{g}=-0.1$ (a) Homoclinic orbit to the saddle point $z_{2}=(-0.2218,0), x_{0}=-0.2218$, (b) $a_{\mathrm{k}}$ versus $\mathrm{k}$, shows the series coefficients converge. 


\subsection{Infinite Series for homoclinic orbits of Equation (3.17)}

Equation (3.17) can be written as

$$
-c \phi+c \phi^{\prime \prime}=4 \phi^{2}-2 \phi \phi^{\prime \prime}-2 \phi^{2}+g
$$

where the ' indicates the derivative with respect to $z$.

Let us consider equilibrium point $z=\left(x_{0}, 0\right)$ of the Equation (3.19) and assume to choose the parameters $\mathrm{c}$ and $\mathrm{g}$ in such a way such that $z=\left(x_{0}, 0\right)$ is a saddle point and a homoclinic orbit to this equilibrium is given (As done in the stability analysis in Chapter 3).

Let us now proceed to construct the homoclinic orbit of Equation (3.17). Suppose for $z>0$

$$
\phi^{+}(z)=\sum_{k=1}^{\infty} a_{k} e^{k \alpha z}+x_{0}
$$

where $\alpha<0$ is an undetermined constant and, $a_{k}, k \geq 1$ are, at the outset, arbitrary coefficients. Substituting the series (4.69) into the Equation (4.68), we obtain the following expressions for each term:

$$
\begin{gathered}
c \phi^{\prime \prime}=c \sum_{k=1}^{\infty} a_{k} k^{2} \alpha^{2} e^{k \alpha z} \\
4 \phi^{2}=4 x_{0}^{2}+8 x_{0} \sum_{k=1}^{\infty} a_{k} e^{k \alpha z}+4 \sum_{k=2}^{\infty} \sum_{i=1}^{k-1} a_{k-i} a_{i} e^{k \alpha z} \\
-2 \phi \phi^{\prime \prime}=-2 x_{0} \sum_{k=1}^{\infty} k^{2} \alpha^{2} a_{k} e^{k \alpha z}-2 \sum_{k=2}^{\infty} \sum_{i=1}^{k-1}(k-i)^{2} \alpha^{2} a_{k-i} a_{i} e^{k \alpha z}
\end{gathered}
$$




$$
-2 \phi^{\prime 2}=-2 \sum_{k=2}^{\infty} \sum_{i=1}^{k-1}(k-i) i \alpha^{2} a_{k-i} a_{i} e^{k \alpha z}
$$

Using Equation (4.69)-(4.73) into the reversible Equation (4.68) we have,

$$
\begin{aligned}
-c x_{0}-4 x_{0}^{2}-g & +\sum_{k=1}^{\infty}\left((k \alpha)^{2}\left(c+2 x_{0}\right)-\left(8 x_{0}+c\right)\right) a_{k} e^{k \alpha z} \\
& =\sum_{k=2}^{\infty} \sum_{i=1}^{k-1}\left[2(k-i) i \alpha^{2}+2(k-i)^{2} \alpha^{2}-3\right] a_{k-i} a_{i} e^{k \alpha z}
\end{aligned}
$$

As $x_{0}$ is an equilibrium of Equation (3.19), $-c x_{0}-4 x_{0}^{2}-g=0$. Comparing the coefficients of $e^{k \alpha z}$ for each $k$, one has for $\mathrm{k}=1$,

$$
\left[\alpha^{2}\left(c+2 x_{0}\right)-8 x_{0}-c\right] a_{1}=0 \text {. }
$$

Assuming $a_{1} \neq 0$ (otherwise $a_{k}=0$ for all $k>1$ by induction), results in the two possible values of $\alpha$ :

$$
\alpha_{1,2}= \pm \sqrt{\frac{8 x_{0}+c}{2 x_{0}+c}}
$$

We are dealing with the case when the equilibrium $x_{0}$ is a saddle, i.e. when $\mathrm{c}<-8 x_{0}$ or $c>-2 x_{0}$. In this case, as our series solution for Equation (4.69) needs to converge for $z>0$, we pick the negative root $\alpha_{2}=-\sqrt{\frac{8 x_{0}+c}{2 x_{0}+c}}$, therefore:

$$
\phi^{+}(z)=\sum_{k=1}^{\infty} a_{k} e^{k \alpha_{2} z}+x_{0}
$$


For $\mathrm{k}=2$ from Equation (4.74) we obtain:

$F\left(2 \alpha_{2}\right) a_{2}=4\left(1-\alpha_{2}^{2}\right) a_{1}^{2}$,

where $F\left(k \alpha_{2}\right)=\left[\left(k \alpha_{2}\right)^{2}\left(c+2 x_{0}\right)-8 x_{0}-c\right]$ and the coefficient $a_{2}$ is easily obtained in terms of $a_{1}$ as,

$$
a_{2}=\frac{4\left(1-\alpha_{2}^{2}\right)}{F\left(2 \alpha_{2}\right)} a_{1}^{2}
$$

For $\mathrm{k}=3$ we obtain from Equation (4.74)

$$
F\left(3 \alpha_{2}\right) a_{3}=2\left(-9 \alpha_{2}^{2}+4\right) a_{1} a_{2}
$$

Once substituted the Equation (4.78) into the Equation (4.79) one obtains $a_{3}$ in terms of $a_{1}$. Thus, for $k>1$

$$
a_{k}=\sum_{i=1}^{k-1} \frac{\left[2(k-i) i \alpha_{2}{ }^{2}+2(k-i)^{2} \alpha_{2}^{2}-3\right] a_{k-i} a_{i}}{F\left(k \alpha_{2}\right)}
$$

Therefore, the coefficients $a_{k}, k>1$ can be directly obtained by iterating the Equation (4.80)

Notice that the Equation (4.68) is reversible, therefore the series solution for $\mathrm{z}<0$ can be easily obtained based on the intrinsic symmetry property of the equation, i.e.:

$$
\phi^{-}(z)=-\sum_{k=1}^{\infty} a_{k} e^{k \alpha_{1} z}+x_{0}
$$

We want to construct a solution of the form: 


$$
\phi(z)= \begin{cases}\phi^{+}(z) & z>0 \\ 0 & z=0 \\ \phi^{-}(z) & z<0\end{cases}
$$

which is continuous at $z=0$, we impose

$$
\phi^{+}(0)=x_{0}+a_{1}+\sum_{k=2}^{\infty} \varphi_{k} a_{1}^{k}=0
$$

Hence, we choose $a_{1}$ as the nontrivial solutions of the following polynomial equations:

$$
x_{0}+a_{1}+\sum_{k=2}^{\infty} \varphi_{k} a_{1}^{k}=0
$$

In practice the Equation (4.83) is numerically solved and the corresponding series solutions are not unique.

Let us now choose $\mathrm{c}=0.5$ and $\mathrm{g}=0.014$. In this case the equation admits two real equilibria: two equilibria $z_{1}=(-0.0827,0)$ and $z_{2}=(-0.0423,0)$. In this parameter regime $\mathrm{z}_{1}$ is a center and $z_{2}$ is a saddle.

Let us build the homoclinic orbit to the saddle point $z_{2}$ (See Figure 8(a)). We find the following continuous solution for the homoclinic orbit. Notice that the Figure 22(a) also shows the travelling nature of the solution. Here $a_{1}=0.0357$. For this choice of the parameters the series solution converges, as shown in the Figure 22(b) where the $a_{k}$ rapidly goes to zero. 

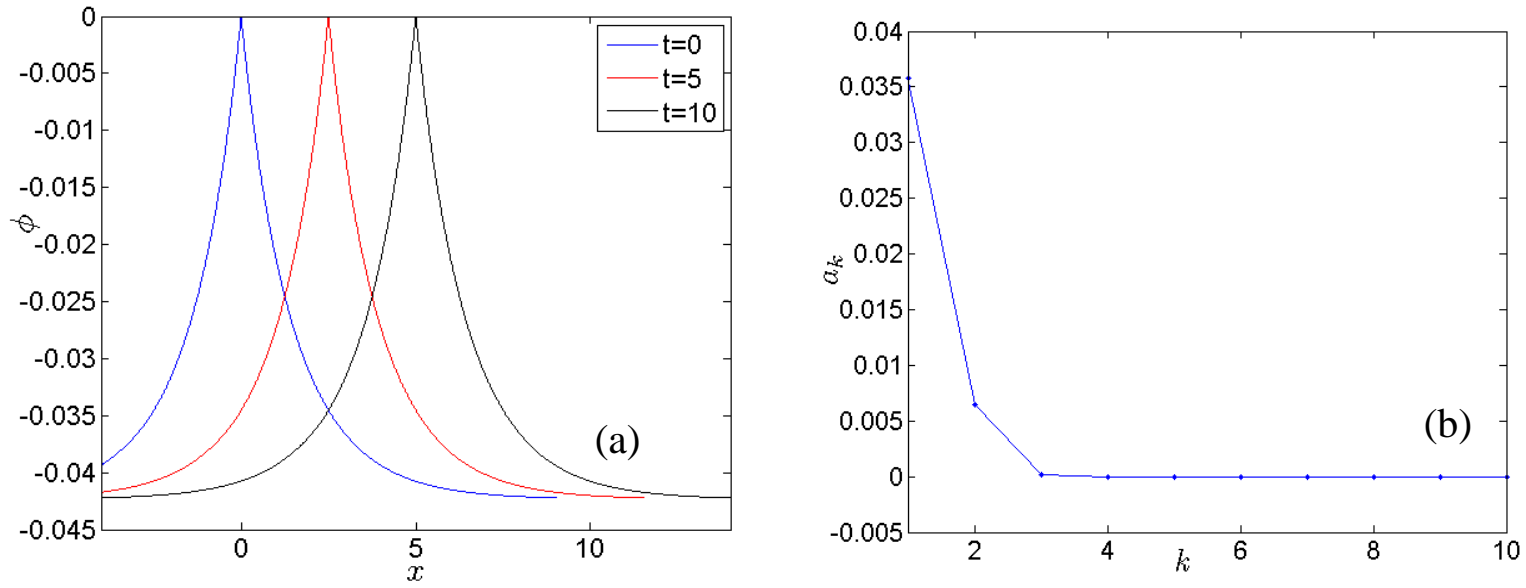

Figure 22: (a) $\phi(x)$ plotted as a function of $x$ for different values of $t$ for $\mathrm{c}=0.5$ and $\mathrm{g}=0.014$ at the equilibrium point $z_{2}$, showing traveling wave nature of the solution Equation (3.17), here $a_{1}=$ 0.0357 (b) Plot of $a_{k}$ versus k shows the series coefficients are converging.
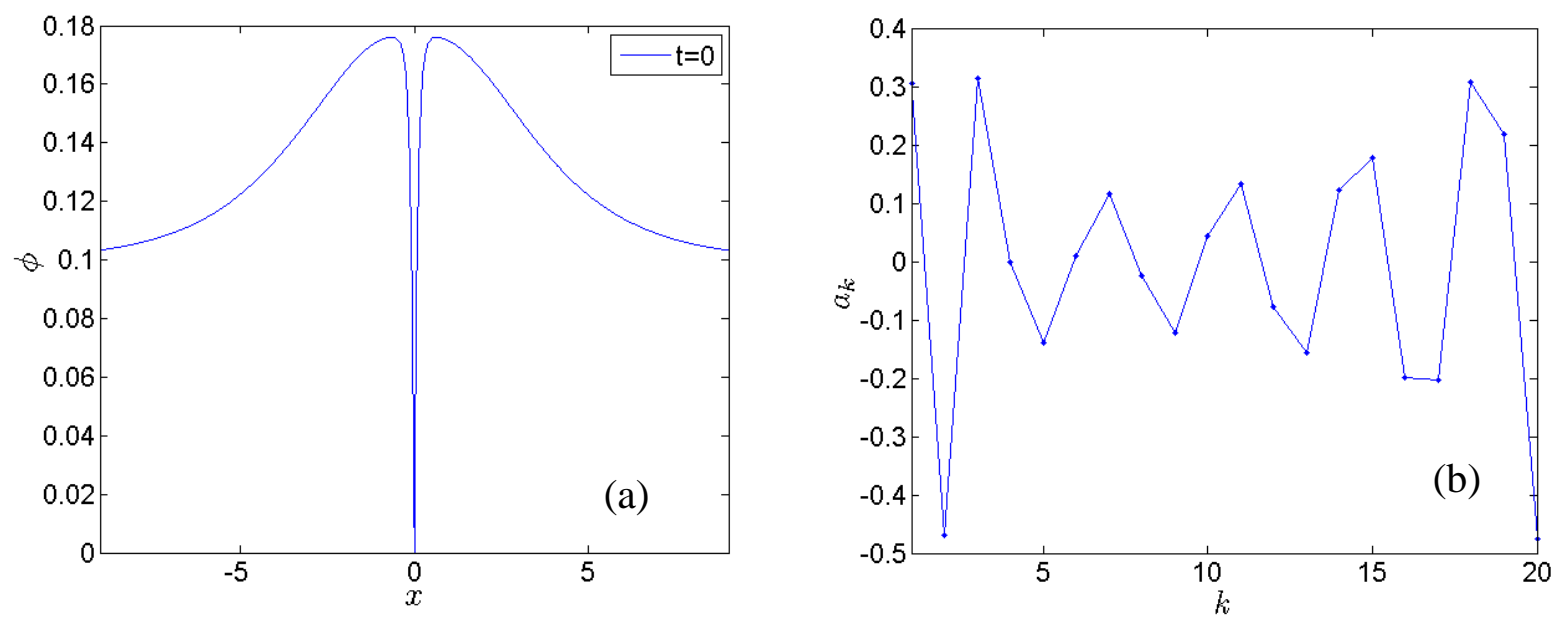

Figure 23: (a) $\phi(x)$ plotted as a function of $x$ for $t=0, \mathrm{c}=-1$ and $\mathrm{g}=0.06$, here $a_{1}=0.3066$ (b) Plot of $a_{\mathrm{k}}$ versus $k$ shows the series coefficients does not converge. 


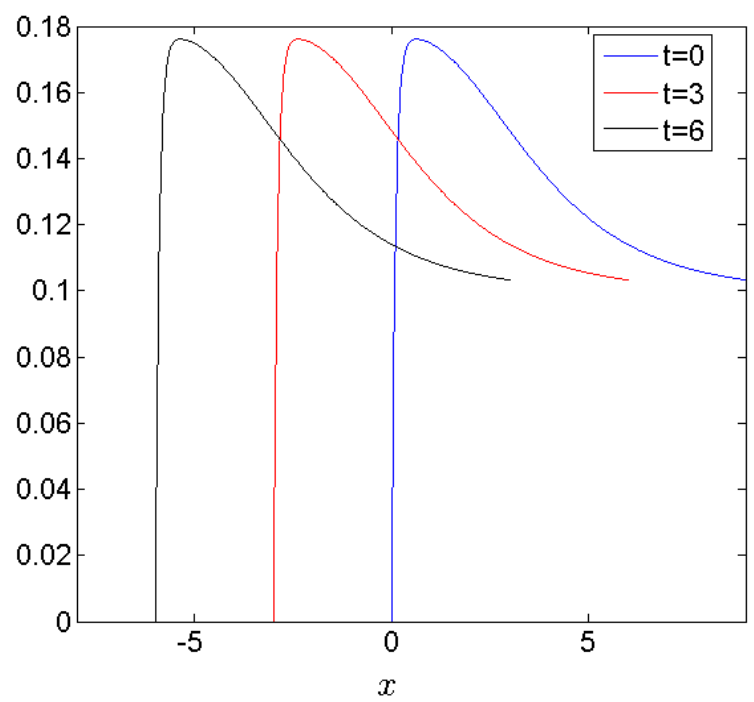

Figure 24: (a) $\phi(x)$ plotted as a function of $x$ for different values of $t$ for $\mathrm{c}=0.5$ and $\mathrm{g}=0.014$ showing traveling wave nature of the solution Equation (3.17).
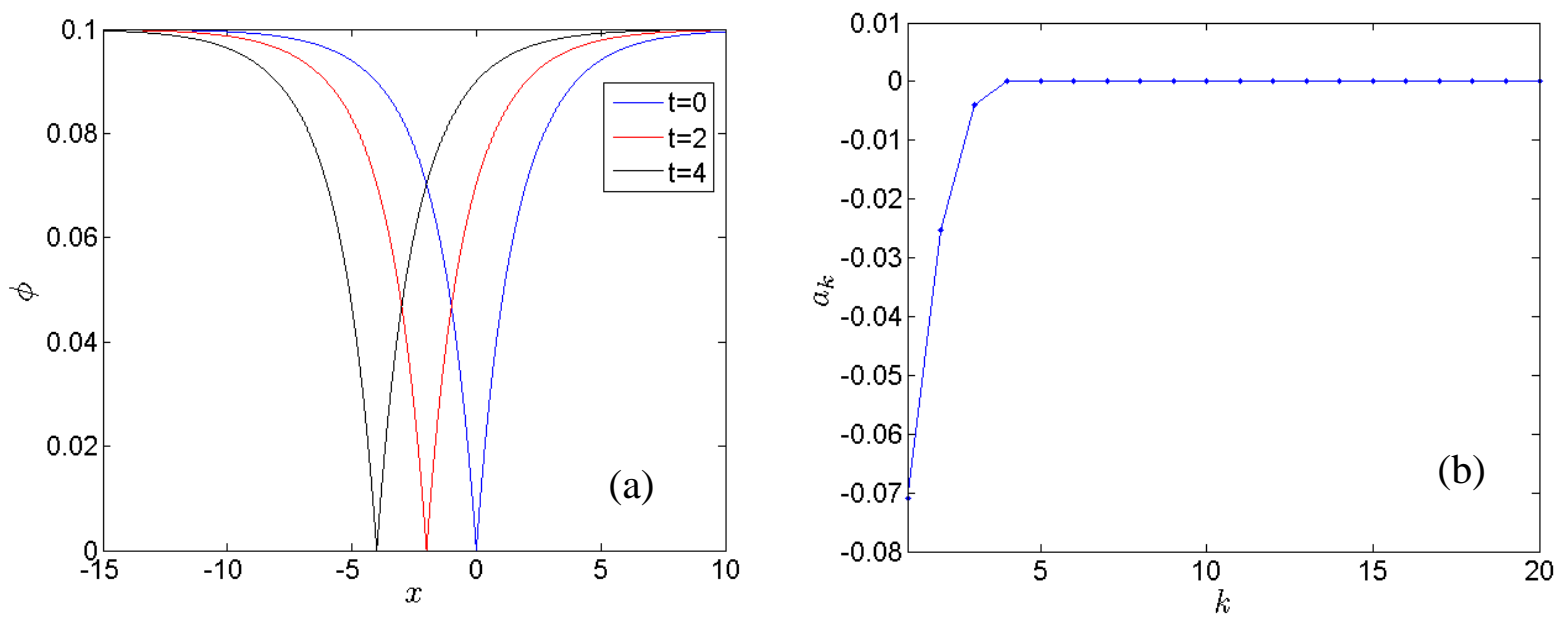

Figure 25: (a) $\phi(x)$ plotted as a function of $\mathrm{x}$ for $\mathrm{t}=0, \mathrm{c}=-1$ and $\mathrm{g}=0.06$, here $a_{1}=-0.0710$. (b) Plot of $a_{k}$ versus $\mathrm{k}$ shows the series coefficients converge. 
To show the travelling nature of the solution (when $\mathrm{c}=.5, \mathrm{~g}=.014$ ) we plot just half part of the solution, for $\mathrm{z}>0$ to make the figure clearer, which is shown in Figure 24 .

Let us consider another numerical example. We choose $c=-1$ and $g=0.06$. In this case the Equation (3.19) admits two real equilibria, the two equilibrium points are $z_{1}=(0.1,0)$ and $z_{2}=(0.15,0)$. In this parameter regime $z_{1}$ is a saddle (with eigenvalues $\alpha_{1,2}= \pm 0.5$ ) and $z_{2}$ is a center.

Let us build the homoclinic orbit to the saddle $\mathrm{z}_{1}$ (See Figure $8(\mathrm{c})$ ). The solution is not unique as the continuity condition admits more than one solution. Choosing $\mathrm{a}_{1}=0.3066$ the series solution has been shown in Figure 23 (a), but the series coefficients $a_{k}$ does not converge.

Choosing $\mathrm{a}_{1}=-0.0710$, both the convergence of the series coefficients and the continuity at the origin have been obtained, as shown in Figure 25. 


\section{CONCLUSIONS}

In this thesis we apply two recent analytical approaches to investigate and analyze possible classes of traveling wave solutions of some members of integrable family of generalized Camassa-Holm $(\mathrm{GCH})$ equations. These equations were recently derived and reported in the literature.

Since we employed a somewhat unusual variant of phase-plane analysis which has been recently developed to consider peakon and cuspon solutions of a wide variety of NLPDEs, we first reviewed it at the beginning of this thesis. There, the dynamics, phase portraits, and wave profiles of $1^{\text {st }}$ and $2^{\text {nd }}$ type of singular traveling waves were discussed in detail.

We chose the following four different $\mathrm{GCH}$ equations:

$$
\begin{aligned}
& \left(1-D_{x}^{2}\right) u_{t}=D_{x}\left(2-D_{x}\right)\left(1+D_{x}\right) u^{2} \\
& \left(1-D_{x}^{2}\right) u_{t}=D_{x}\left(2+D_{x}\right)\left[\left(2-D_{x}\right) u\right]^{2} \\
& \left(1-D_{x}^{2}\right) u_{t}=D_{x}\left(u^{2} u_{x x}-u_{x}^{2} u_{x x}+u u_{x}^{2}-u^{3}\right) \\
& \left(1-D_{x}^{2}\right) u_{t}=D_{x}\left(4-D_{x}^{2}\right) u^{2}
\end{aligned}
$$

and

and the novel application of phase-plane analysis Chapter 2 was applied to predict the possible occurrence of non-smooth peakon, cuspon, and compacton solutions. 
For the first two equations, the phase portraits were drawn and investigated for different conditions and values of $\mathrm{g}$ and $\mathrm{c}$. We found that both the equations did not produce any closed curve triangles, arched curves or oval curves near the singular planar curve. Therefore for these two equations, neither peakon nor cuspons are possible. We generalized an existing theorem to establish the existence of peakon solutions of the third GCH equation. This equation was found to also support four segmented, non-smooth M-wave solutions. From the theorems in Chapter 2, the fourth GCH equation was found to support both solitary (peakon) and periodic (cuspon) cusp waves in different parameter regimes.

In the second part of the thesis, smooth traveling wave solutions of the above four GCH equations were considered. Here, we used a recent technique to derive convergent multi-infinite series solutions for the homoclinic and heteroclinic orbits of their traveling-wave equations, corresponding to pulse and front (kink or shock) solutions respectively of the original PDEs.

We performed many numerical tests in different parameter regime to pinpoint real saddle equilibrium points of the corresponding GCH equations. This ensured simultaneous convergence and continuity of the multi-infinite series solutions for the homoclinic and heteroclinic orbits. We have also shown the traveling wave nature of these GCH equations.

Unlike the majority of unaccelerated convergent series, high accuracy is attained with relatively few terms of our series. Of course, the convergence rate is not comparable to typical asymptotic series. However, asymptotic solutions for global behavior along a full homoclinic/heteroclinic orbit are currently not available. 


\section{REFERENCES}

[1] R. Camassa, and D. Holm, "An integrable shallow water equation with peaked solitons," Phys. Rev. Lett. 71, 1661 (1993). R. Camassa, D. Holm, and J. Hyman, “A new integrable shallow water equation," Adv. Appl. Mech 31, 1 (1994).

[2] R. S. Johnson, "On the solutions of the Camassa-Holm equation," Proc. R. Soc. Lond. A 459, 1687 (2003). R. S. Johnson, “Camassa-Holm, Korteweg-de Vries and related models for water waves," J. Fluid Mech 455, 63 (2002).

[3] A. Degasperis, D. D. Holm, and A. N. W. Hone, "A new integrable equation with peakon solutions," Theoretical and Mathematical Physics 133, 1463 (2002).

[4] V. Novikov, "Generalization of the Camassa-Holm equation," J. Phys.A: Math. Theor.42, $342002(2009)$.

[5] A. V. Mikhailov and V. S. Novikov, "Perturbative symmetry approach," J. Phys. A: Math. Gen. 35, 4775 (2002).

[6] A. N. W. Hone and J. P. Wang, "Integrable peakon equations with cubic nonlinearity," J. Phys. A: Math. Theor. 41, 372002 (2008).

[7] J. Li and H. Dai, "On the study of singular nonlinear travelling wave equations: dynamical approach[M]," Beijing: Science Press, $2^{\text {nd }}$ edition (2007).

[8] S. R. Choudhury and G. Gambino, “ Convergent analytic solutions for homoclinic orbits in reversible and non-reversible systems," (To appear in Nonlinear Dynamics, published online April 2013)

[9] X. Wang, "Si'lnikov chaos and Hopf bifurcation analysis of Rucklidge system," Chaos, Solitons and Fractals 42, 2208 (2009). 
[10] C. David and Z. Feng (Ed.) "Solitary waves in fluid media," Ch. 7, pp. 123-201 by J. Li, Bentham Science, Sharjah (2010)

[11] A. R. Champneys, "Homoclinic orbits in reversible systems and their application in mechanics, fluids and optics," Physica D 112, 158 (1998).

[12] Y. A. Kuznetsov, "Elements of Applied Bifurcation Theory," Springer-Verlag, New York, (1995).

[13] P. Glendinning, "Stability, Instablity, and Chaos," Cambridge Univ. Press, Cambridge, (1994). 